\title{
Search for Popcorn Mesons in Events with Two Charmed Baryons
}

\author{
Brandon Hartfiel \\ Stanford Linear Accelerator Center \\ Stanford University \\ Stanford, CA 94309
}

SLAC-Report-823

Prepared for the Department of Energy

under contract number DE-AC02-76SF00515

Printed in the United States of America. Available from the National Technical Information Service, U.S. Department of Commerce, 5285 Port Royal Road, Springfield, VA 22161. 
This document, and the material and data contained therein, was developed under sponsorship of the United States Government. Neither the United States nor the Department of Energy, nor the Leland Stanford Junior University, nor their employees, nor their respective contractors, subcontractors, or their employees, makes an warranty, express or implied, or assumes any liability of responsibility for accuracy, completeness or usefulness of any information, apparatus, product or process disclosed, or represents that its use will not infringe privately owned rights. Mention of any product, its manufacturer, or suppliers shall not, nor is it intended to, imply approval, disapproval, or fitness of any particular use. A royalty-free, nonexclusive right to use and disseminate same of whatsoever, is expressly reserved to the United States and the University. 


\title{
Search for Popcorn Mesons in Events with Two Charmed Baryons
}

\author{
A DISSERTATION \\ SUBMITTED TO THE DEPARMENT OF PHYSICS \\ AND THE COMMITTEE ON GRADUATE STUDIES \\ OF UNIVERSITY OF CALIFORNIA, LOS ANGELES \\ IN PARTIAL FULFILLMENT OF THE REQUIREMENTS \\ FOR THE DEGREE OF \\ DOCTOR OF PHILOSOPHY
}

Brandon Hartfiel

June 2005 
SLAC-R-823

May 2006

\title{
Search for Popcorn Mesons in Events with Two Charmed
}

\author{
Baryons* \\ Brandon Hartfiel \\ Stanford Linear Accelerator Center, Stanford University, Stanford CA 94309
}

\begin{abstract}
The physics of this note is divided into two parts. The first part measures the $\Lambda_{c} \rightarrow \pi k p$ continuum momentum spectrum at a center of mass energy of $10.54 \mathrm{GeV} / \mathrm{c}$. The data sample consists of $15,400 \Lambda_{c}$ baryons from $9.46 \mathrm{fb}^{-1}$ of integrated luminosity. With more than 13 times more data than the best previous measurement, we are able to exclude some of the simpler, one parameter fragmentation functions.

In the second part, we add the $\Lambda_{c} \rightarrow K^{0} p$ mode, and look for events with a $\Lambda_{c}^{+}$and a $\bar{\Lambda}_{c}^{-}$in order to look for "popcorn" mesons formed between the baryon and antibaryon. We add on-resonance data, with a kinematic cut to eliminate background from B decays, as well as BaBar run 3 and 4 data to increase the total data size to $219.70 \mathrm{fb}^{-1}$. We find 619 events after background subtraction. After a subtraction of $1.06 \pm .09$ charged pions coming from decays of known resonances to $\Lambda_{c}+n \pi$, we are left with $2.63 \pm .21$ additional charged pions in each of these events. This is significantly higher than the .5 popcorn mesons per bayon pair
\end{abstract}

${ }^{*}$ Work supported by Department of Energy contract DE-AC02-76SF00515 
used in the current tuning of Pythia 6.2, the most widely used Monte Carlo generator.

The extra mesons we find appear to be the first direct evidence of popcorn mesons, although some of them could be arising from hypothetical unresolved, unobserved charmed baryon resonances contributing decay mesons to our data. To contribute a significant fraction, this hypothesis requires a large number of such broad unresolved states and seems unlikely, but can not be completely excluded. 


\section{TABle of Contents}

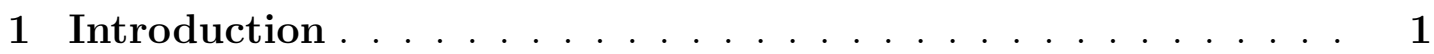

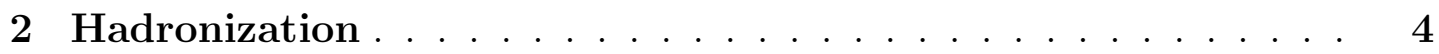

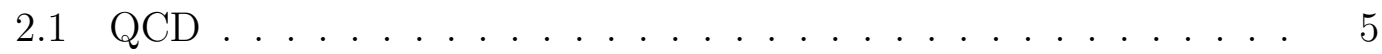

2.2 Hadronization Models . . . . . . . . . . . . . . . . . . 8

2.2.1 The Lund Model and Fragmentation Function . . . . . . 9

2.2 .2 Other Fragmentation Functions . . . . . . . . . . . . 14

2.2 .3 The UCLA Model . . . . . . . . . . . . . . . . . . . . . 17

2.3 Baryon Formation . . . . . . . . . . . . . . . . . . . . 21

2.3.1 Baryons in the Lund Model . . . . . . . . . . . . . . 24

2.3.2 Baryons in the UCLA model . . . . . . . . . . . . 26

2.3.3 Previous Evidence for Popcorn _ . . . . . . . . . . 28

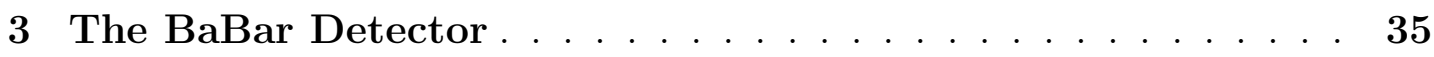

3.1 Detector Asymmetry . . . . . . . . . . . . . . . . 35

$3.2 \Upsilon(4 S)$ and Continuum Events . . . . . . . . . . . . 35

3.3 Silicon Vertex Tracker (SVT) . . . . . . . . . . . . . 36

3.4 Drift Chamber $(\mathrm{DCH}) \ldots \ldots \ldots \ldots$

3.5 Detector of Internally Reflected Cherenkov Radiation (DIRC) . . 37

3.6 Electromagnetic Calorimeter (EMC) $\ldots \ldots \ldots \ldots$

3.7 Instrumented Flux Return (IFR) $\ldots \ldots \ldots \ldots$ 
4 Particle Identification . . . . . . . . . . . . . . . . . . . 39

4.1 Charged Particles . . . . . . . . . . . . . . . . . . . . . . . 39

4.1.1 DCH and DIRC Likelihoods . . . . . . . . . . . . 39

4.1 .2 Optimization Method . . . . . . . . . . . . . . . . 42

4.1 .3 Performance . . . . . . . . . . . . . . . . . 46

4.2 Neutral Particles . . . . . . . . . . . . . . . . . . . . . . 49

5 Reconstruction of $\Lambda_{c}$ 's $\ldots \ldots \ldots \ldots \ldots \ldots$

5.1 Event and Track Selection $\ldots \ldots \ldots \ldots$. . . . . . . . . 51

5.2 Lab Frame Efficiency Map . . . . . . . . . . . . . . . . . . 53

5.2 .1 Making the Efficiency Map . . . . . . . . . . . . . . 56

$5.2 .2 \quad$ Data/MC corrections . . . . . . . . . . . . . . . 61

6 Measurement of the $\Lambda_{c}$ Momentum Spectrum $\ldots \ldots \ldots 63$

6.1 Signal Extraction . . . . . . . . . . . . . . . . . . 63

6.1 .1 Full Dataset Study . . . . . . . . . . . . . . . . . . . . 64

6.1 .2 Fitting the Offpeak Data . . . . . . . . . . . . . 66

6.2 Systematic Errors . . . . . . . . . . . . . . . . . . . . . 68

6.2 .1 Reflections . . . . . . . . . . . . . . . . . 68

6.2.2 Momentum Resolution and Shift _. . . . . . . . 76

6.2.3 Resonant Substructure of Decays _ . . . . . . . . . 78

6.2 .4 Proton Helicity Angle . . . . . . . . . . . . . . . . . 80

6.2.5 MC/Data Momentum Spectrum Shift . . . . . . . . . 80

6.2.6 Angular Distribution in Monte Carlo and Data . . . . . 82 
6.2.7 Time Dependance . . . . . . . . . . . . . . 84

6.3 Results........................ 86

7 Comparisons with Previous Experiments and Models . . . . . 93

$8 \Lambda_{c}^{+} \mid \bar{\Lambda}_{c}^{-}$Events ........................ 103

8.1 Selection of $\Lambda_{c}^{+} \mid \bar{\Lambda}_{c}^{-}$Events . . . . . . . . . . . . . . . 103

8.2 Four Baryon Events . . . . . . . . . . . . . . . . 113

8.3 Decays of Heavier Charmed Baryons . . . . . . . . . . . . . 115

8.4 Efficiency Corrections . . . . . . . . . . . . . . 122

$8.5 \quad K_{0}, \rho$ and $\omega \ldots \ldots \ldots \ldots . \ldots \ldots \ldots$

8.6 Results . . . . . . . . . . . . . . . . . . . . . . . 128

8.7 Decays of Undiscovered Charmed Baryons. . . . . . . . . . . . 133

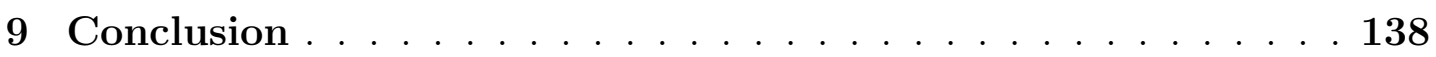

References ....................... 141 


\section{CHAPTER 1}

\section{Introduction}

When a quark and antiquark move apart at ultrarelativistic velocities, their kinetic energies are seen to be converted into new particles. This process is known as hadronization. The new particles can be classified into two types: mesons, which contain two quarks, and baryons, which contain three quarks.

Because the sum of the quantum numbers of the constituents of the final state particles must be the same as in the original quark-antiquark pair, any newly created quarks must arrive in matter-antimatter pairs. Since quarks are created in pairs and baryons contain an odd number of quarks, we will always see an even number of baryons in any hadronization reaction. In fact, we will have an equal number of baryons and antibaryons.

Reactions resulting only in final state mesons can be described as coming from a series of newly created quark-antiquark pairs arranged in a string like geometry along the line of the original quark-antiquark relative velocity. Each member of a newly created quark-antiquark pair joins up with the quark or antiquark from a neighboring pair, forming a series of mesons which can be reconstructed from detector data.

When a baryon-antibaryon pair is formed, the situation is more complicated. Baryons can form adjacent to each other in the chain, or they can be formed with one or more mesons in between them. In the second case, the swapping of 
partners inside the chain requires longer flight lengths of virtual quarks, which will lead to a suppression factor. This will be explained in more detail in chapter 2 .

The mesons formed in the middle of the baryon-antibaryon pair are known as popcorn mesons. Popcorn mesons were originally proposed as a way to explain the absence of strong flavor correlations in baryon-antibaryons pairs. Studies attempting to find mesons in the rapidity gap between baryon-antibaryon pairs however, have not been able to give conclusive results.

In 2001, the CLEO collaboration noticed, in $10.6 \mathrm{GeV}$ electron-positron collisions resulting in a jetlike event with both a $\Lambda_{c}^{+}$and a ${\overline{\Lambda_{c}}}^{-}$, that no other baryons were observed in the event [1]. This is an interesting result because the $\Lambda_{c}{ }^{*}$ are always seen to move nearly back to back, but the current Monte Carlo generators are unable to create correlated baryons in opposite jets.

This type of event geometry is the perfect place to look for popcorn mesons, since we have a baryon-antibaryon pair with a large rapidity difference, and nothing but mesons in the rest of the event. The only background we have to worry about is the decay products of excited charmed baryons going to a $\Lambda_{c}+$ pions. Of course, the large rapidity difference of leading $\Lambda_{c}^{+}$and a $\bar{\Lambda}_{c}{ }^{-}$might bias our result. We might expect some kind of scaling to occur for more typical events with smaller baryon-antibaryon rapidity differences.

Before searching for popcorn, we will first measure the momentum spectrum of individual $\Lambda_{c}$ baryons in jetlike events. This is also a new area of research since most of the fragmentation functions used to describe the momentum spectrum were specifically designed to model meson production, and the previous best measurement of the $\Lambda_{c}$ momentum spectrum used only 1/13 as much data.

This thesis is organised as follows. Chapter 2 reviews quantum chromody-

\footnotetext{
*unless stated otherwise, charge conjugation is implied throughout this thesis
} 
namics and provides an introduction to the various hadronization models, which are used to describe the $\Lambda_{c}$ momentum spectrum. Unfortunately, none of the current implementations of the models are able to create events with two $\Lambda_{c}$ and no other baryons.

Chapter 3 gives a short description of the BaBar detector, where the data for this analysis were collected. Chapter 4 describes how information from BaBar's drift chamber and Cherenkov detector are combined to identify charged particles over a wide momentum range. It also discusses how neutral $\Lambda$ and $K_{s}^{0}$ particles are identified. Chapter 5 describes how charged pions, kaons and protons are reconstructed in order to make the continuum spectrum measurement of $\Lambda_{c} \rightarrow$ $\pi K p$. The actual measurement and various cross checks are made in chapter 6 along with an estimation of the systematic errors. In chapter 7 we compare our result to the previous CLEO measurement and to the various hadronization models. Finally in chapter 8, we search for popcorn mesons in events which contain both a $\Lambda_{c}^{+}$and a $\bar{\Lambda}_{c}^{-}$, with conclusions in Chapter 9. 


\section{CHAPTER 2}

\section{Hadronization}

When we collide high energy beams of electrons and positrons, we often see the

reaction $e^{+} e^{-} \rightarrow q \bar{q}$. As the quark-antiquark pair move apart, it is believed that the strong force between them eventually creates a linear potential with an energy density of the order of $1 \mathrm{GeV}$ per fermi. New quark-antiquark pairs are created from this energy and rearrange themselves into mesons and baryons which are concentrated into back to back jets aligned approximately along the original $q \bar{q}$ flight direction. This process, know as hadronization, is responsible for a large fraction of the multiparticle events seen in $e^{+} e^{-}$collisions.

The early stages of hadronization, which determine the topology of the event - the thrust, planarity etc. of the spatial distribution of final particles - can be described by perturbative Quantum Chromodynamics (QCD). However, as more particles are created, the typical momentum transfer drops, increasing the QCD coupling constant to the point where perturbative calculations are no longer possible. It is in these later stages of hadronization that the types of final state particles and their momentum correlations are determined. Here we must rely on phenomenological models, which will be described in this chapter, along with the results of some previous hadronization experiments. This thesis is a part of the programatic progress in understanding this process. 


\section{$2.1 \quad$ QCD}

The strong forces responsible for hadronization are described by Quantum Chromodynamics (QCD), which, like electroweak theory, is based on gauge invariance [2]. The force-carrying particles, know as gluons, come about by starting with a free quark Lagrangian and then adding new terms in a way that makes the total Lagrangian invariant to special unitary transformations of color. The free quark Lagrangian is

$$
\mathcal{L}_{0}=\bar{q}\left(i \gamma^{\mu} \partial_{\mu}-m\right) q
$$

where $\mathrm{q}$ is a three component vector describing the red, blue and green components of the quark.

$$
q=\left(\begin{array}{l}
r \\
b \\
g
\end{array}\right)
$$

Unitary transformations change the amounts of redness, blueness and greenness and their phases while keeping $|r|^{2}+|b|^{2}+|c|^{2}$ constant. Special unitary transformations are the set of all unitary transformations except those that globally change the phases of all three colors by the same amount. They can be written as

$$
U=e^{i \alpha_{a}(x) \lambda_{a}}
$$

where $\alpha_{a}$ are arbitray angles of rotation and $\lambda_{a}$ are the eight hermitian matrices shown in table 2.1. The transformations described by these matrices, known as the Gell-Mann matrices, will correspond to the eight gluons.

Later we will need notation for the commutators of these matrices

$$
\left[\lambda_{\alpha}, \lambda_{\beta}\right]=2 f_{\alpha \beta \gamma} \lambda_{\gamma}
$$




$$
\begin{aligned}
& \lambda_{1}=\left(\begin{array}{lll}
0 & 1 & 0 \\
1 & 0 & 0 \\
0 & 0 & 0
\end{array}\right) \quad \lambda_{2}=\left(\begin{array}{rrr}
0 & -i & 0 \\
i & 0 & 0 \\
0 & 0 & 0
\end{array}\right) \quad \lambda_{3}=\left(\begin{array}{rrr}
1 & 0 & 0 \\
0 & -1 & 0 \\
0 & 0 & 0
\end{array}\right) \\
& \lambda_{4}=\left(\begin{array}{ccc}
0 & 0 & 1 \\
0 & 0 & 0 \\
1 & 0 & 0
\end{array}\right) \quad \lambda_{5}=\left(\begin{array}{ccc}
0 & 0 & -i \\
0 & 0 & 0 \\
i & 0 & 0
\end{array}\right) \quad \lambda_{6}=\left(\begin{array}{ccc}
0 & 0 & 0 \\
0 & 0 & 1 \\
0 & 1 & 0
\end{array}\right) \\
& \lambda_{7}=\left(\begin{array}{rrr}
0 & 0 & 0 \\
0 & 0 & -i \\
0 & i & 0
\end{array}\right) \quad \lambda_{8}=\frac{1}{\sqrt{3}}\left(\begin{array}{rrr}
1 & 0 & 0 \\
0 & 1 & 0 \\
0 & 0 & -2
\end{array}\right)
\end{aligned}
$$

Table 2.1: Eight Gell-Mann matrices

Now, lets see how an infinitesimal special unitary transformation changes $\mathcal{L}_{0}$. The color vector and derivative change in the following ways

$$
\begin{gathered}
q(x) \Rightarrow\left[1+i \alpha_{a}(x) \lambda_{a}\right] q(x) \\
\partial_{\mu} q(x) \Rightarrow\left[1+i \alpha_{a}(x) \lambda_{a}\right] q(x)+i \lambda_{a} q(x) \partial_{\mu} \alpha_{a}(x) .
\end{gathered}
$$

The mass term in the free Lagrangian (2.1), is unchanged by this transformation, but the derivative of $\alpha_{a}$ in (2.2) must be compensated in some way in order to preserve the invariance of the Lagrangian. This is done by replacing the derivative in (2.1) by a covariant derivative of the form

$$
D_{\mu}=\partial_{\mu}+i g \lambda_{a} G_{\mu}^{a}
$$


where $G_{\mu}^{a}$ are the new gluon fields which transform like

$$
G_{\mu}^{a} \Rightarrow G_{\mu}^{a}-\frac{1}{g}+\partial_{\mu} \alpha_{a}-f_{a b c} \alpha_{b} G_{\mu}^{c}
$$

and $\mathrm{g}$ is a model parameter to be determined by experiment $\left(\alpha_{s}=\frac{g^{2}}{4 \pi}\right)$. These gluon fields will need to have a kinetic energy term, which, as in the case of the photon, is proportional to the contraction of the field strength tensor, $G_{\mu \nu}^{a}$. The field strength tensor is defined by

$$
\left[D_{\mu}, D_{\nu}\right]=i g \lambda_{a} G_{\mu \nu}^{a}
$$

Now, our total Lagrangian is

$$
\mathcal{L}=\bar{q}\left(i \gamma^{\mu} \partial_{\mu}-m\right) q-g\left(\bar{q} \gamma^{\mu} \lambda_{a} q\right) G_{\mu}^{a}-\frac{1}{4} G_{\mu \nu}^{a} G_{a}^{\mu \nu}
$$

where

$$
G_{\mu \nu}^{a}=\partial_{\mu} G_{\nu}^{a}-\partial_{n} u G_{\mu}^{a}-g f_{a b c} G_{\mu}^{b} G_{\nu}^{c}
$$

The $f_{a b c}$ term in (2.4) is due to the non-abelian nature of the Gell-Mann matrices. When substituted into (2.3), this term leads to gluon-gluon interactions which will show up as 3-gluon and 4-gluon vertices in Feynman diagrams. The non-abelian term causes the gluons exchanged between quarks to confine themselves in a narrow tube. This result will be used as the starting point for the string model of hadronization described in the next section.

The existence of a 3-gluon vertex also means that the Feynman diagrams will contain gluon loops, which will modify the renormalization procedure which leads to the running coupling constant. To first order, the coupling constant is

$$
\alpha_{s}\left(Q^{2}\right)=\frac{\alpha_{s}\left(\mu^{2}\right)}{1-k \log \left(\frac{Q^{2}}{\mu^{2}}\right)}
$$


where $\mu$ is a reference momentum, and $\mathrm{k}$ is $\frac{1}{3 \pi}$ for QED and $-\frac{7}{4 \pi}$ for QCD. The change in sign of $\mathrm{k}$ means that for QCD the coupling will be high for low momentum transfers. The current PDG value for $\alpha_{s}$ at $665 \mathrm{MeV}$ is $1.0+0.4-$ 0.2. Since the momentum transfers inside of hadrons will be much lower than 665 $\mathrm{MeV}$, we will not be able to use perturbation theory to describe the hadronization process, and we will have to turn to the phenomenological models described in the next section.

\subsection{Hadronization Models}

The transition $e^{+} e^{-} \rightarrow$ multiple hadrons can be divided into two stages based on the value of the QCD coupling constant $\alpha_{s}\left(Q^{2}\right)$. In the early stages, gluon brehmstrahlung and the subsequent splitting of gluons into quark-antiquark pairs can be described by Alterelli-Parisi type equations. This parton shower stage typically continues until the virtual masses of the quarks and gluons fall below about $2 \mathrm{GeV}$. At this point, perturbative calculations are no longer effective and we must come up with a way of modeling the soft gluon emissions and the subsequent coalesecence of the partons into stable hadrons and resonances.

Here, we are guided by lattice QCD results [3] which show that the energy density of the partons created between the primary quarks is confined to a narrow tube, or string, of a constant radius of about half a fermi. The hard gluons from the parton shower are modeled by kinks in the string, which are responsible for the appearance of 3-jet events and also a component of the final particles' transverse momenta with respect to the primary quarks. Our primary concern in this paper, however, is the flavor and longitudinal momentum of the final particles. The string phenomenology allows us to concentrate on these two aspects by collapsing the problem of hadronization into one dimension. 


\subsubsection{The Lund Model and Fragmentation Function}

One of the challenges for hadronization models is to correctly predict the multiplicities of hadrons of different flavors. Table 2.4, which gives a summary of the meson multiplicities at the end of this chapter, shows that the charged kaon multiplicity in $10 \mathrm{GeV} e^{+} e^{-} \rightarrow$ multiple hadrons events is less than one seventh of the charged pion mulitplicity. The Lund model [4], the basis of the most widely used Monte Carlo program for high energy collisions, explains this as being due to the tunneling of massive quarks out of the linear potential of the string.

We can calculate the rate of tunneling by considering the time-reverse process, which will have the same amplitude. A quark and an antiquark, each with total energy zero approach each other in a linear potential. At any point, the kinetic energy of each quark is equal to $k x-m$, where $k$ is the energy density of the string, about $1 \mathrm{GeV} /$ fermi, and $x$ is the distance from the center of mass. Classically the quarks will rebound at $x= \pm m / k$, but the quantum WKB approximation shows that they will annihilate with probability

$$
P_{q}=\exp \left[-\left(\frac{\pi m_{q}^{2}}{k}\right)\right]
$$

which is identical to the probability of the time reversed process of quarks tunneling out of the potential*. Theoretically, the light quark masses are not well defined. The Lund model tunes the parameter $P_{s} / P_{u}$ to fit the hadronization data, and we can work backwards from its value of 0.3 to get a strange quark mass of $275 \mathrm{MeV}$, which is reasonable, and could be more precisely tuned by changing $\mathrm{k}$.

Using this approach, the c quark mass of $1100 \mathrm{MeV}$ corresponds to a tunneling probability of $10^{-9}$ of that for a light quark. In practice, c and heavier quarks are

\footnotetext{
${ }^{*}$ It is conventional to leave out the $\mathrm{c}$ and $\hbar$ in these equations.
} 
never created from the string in the JETSET algorithm. We will see in chapter 8 that non-leading c quarks show up very rarely in the data.

In order for tunneling to lead to the production of independent hadrons from the string, the new quarks must form color singlets $\left(\frac{1}{\sqrt{3}}(r \bar{r}+b \bar{b}+g \bar{g})\right)$ with the original quarks. In this configuration, the new quarks shield the central region of the event from the color fluxes emanating from the original quark-antiquark pair. We now have two smaller quark-antiquark color singlet systems which can again be split by the tunneling of new quark-antiquark pairs. The iterative splitting of color flux tubes leads to a chain of mesons with local flavor conservation.

In section 2.3 we will see that if the tunneling quarks form a color antitriplet state with the original quarks, baryon formation may occur. Simple first order QCD calculations show that the color octet and sextet configurations are repulsive [5] and will not lead to the formation of hadrons.

Another experimental fact that the hadronization models must account for is the suppression of vector particles, compared to pseudo-scalar particles. Here table 2.4 can be misleading because a larger percentage of the pseudoscalar particles come from the decay of heavier particles and not directly from the string. We can use decay tables to work backwards and calculate the direct production rates and we see that vector particles are indeed suppressed. The Lund model explains this by noticing that adding a spin-spin term to the Hamiltonian will modify the shape of the wavefunctions in the annihilation region and change the tunneling probability. Phenomenologically the model uses the following values

$$
\frac{P_{\rho}}{P_{\rho}+P_{\pi}}=0.5 \quad \frac{P_{K^{*}}}{P_{K^{*}}+P_{K}}=0.6 \quad \frac{P_{D^{*}}}{P_{D^{*}}+P_{D}}=0.75
$$

where $\mathrm{P}$ is the probability of a particular particle being formed. It is interesting to note that as $M_{v} / M_{s} \rightarrow 1, P_{v} / P_{s} \rightarrow 3$ which is what we expect from simple spin counting. 
The JETSET model has other tunable parameters [4] that we will not need to discuss here.

Now that we have figured out the probabilities of creating different types of hadrons, we need to make a procedure for determining their momenta. It is useful at this point to look at a space-time diagram of the string breaking process. Figure 2.1 shows the original quark-antiquark pair being created at "a", and subsequent pairs tunneling out of the string at "b" and "c". After these points the mesons continue on in "yo-yo mode". The energy of each meson oscillates between the linear potential of about $1 \mathrm{GeV} /$ fermi of the string, and the kinetic energies of the quark-antiquark pair. Thus, the energy of the meson in $\mathrm{GeV}$ is equal to the distance, in fermi, between the two widest points in one period and the momentum is equal to the time difference between these two points. The mass of the meson is equal to the square root of two times the space-time area swept out over one period [6].

The interior quarks will almost always be light ( $\mathrm{u}, \mathrm{d}$ or $\mathrm{s})$, and thus will move at ultra-relativistic velocities represented by 45 degree lines on the space-time diagram. This means that points "b" and "c" will not be causually connected. Thus, if we want to describe the breaking of the string as an iterative process, we must do so in a way that is independent of the time ordering of the breaks. This requirement leads to the Lund Symmetric Fragmentation Function

$$
f(z)=\frac{1}{z}(1-z)^{a} \exp \left(-b m_{\perp}^{2} / z\right)
$$

where

$$
z=\frac{\left(E+P_{z}\right)_{\text {hadron }}}{\left(E+P_{z}\right)_{\text {quark }}}
$$

and $m_{\perp}$ is the transverse mass $\sqrt{P_{T}^{2}+m^{2}}$, which will usually be referred to 


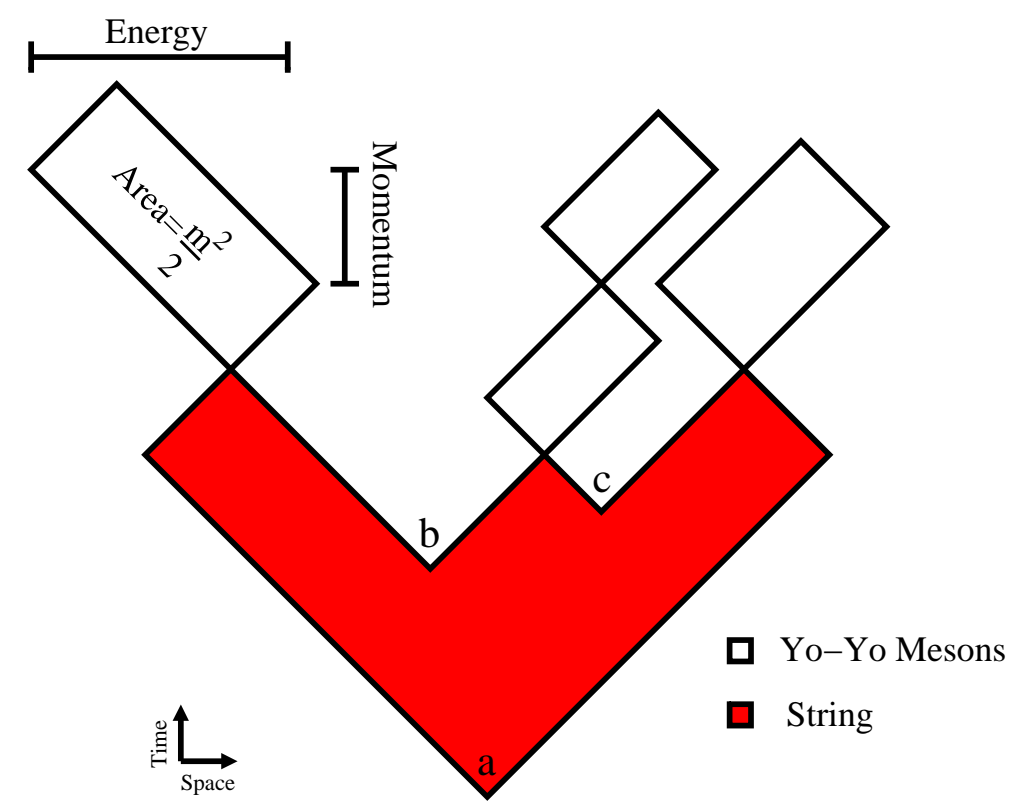

Figure 2.1: A string fragmenting into 3 mesons. Because the energy density is about $1 \mathrm{GeV} / \mathrm{fm}$ it is easy to convert distances into energy and momentum. 
simply as "mass" in the rest of this chapter, and "a" and "b" are phenomenological parameters, which will be tuned to the data.

Another interesting feature of this function is that it results in a rapidity space distibution of particles which is flat except where it drops off near the kinematic boundaries.

Now we have a complete algorithm for modeling the hadron flavors and longitudinal momenta in $e^{+} e^{-} \rightarrow q \bar{q}$ in an "outside-in" iterative implementation, beginning with the outermost, primary quark and antiquark pair created by the virtual photon. First the type of hadron is determined using a set of probabilities based on the quark content and other properties of the different hadrons. Then the longitudinal momentum is determined by plugging the hadron mass into the fragmentation function. The process is then iterated inward, one hadron at a time.

There is a small implementation problem, however, caused by the requirement that the last hadron formed must have exactly the energy and mass left over in the system after the creation of the rest of the event. When the Monte Carlo generator reaches the point where there is less than about $2 \mathrm{GeV}$ remaining in the string, a separate algorithm choses the final two central particles in a way that makes the quantum numbers of the two sides match, while conserving energy and momenta. An unfortunate consequence of this is that baryon number is conservered in each jet separately, and thus events of the type seen in chapter 8 cannot be modeled. This will not, however, affect our ability to compare the model's quantitative predictions to data, because we know how the model is supposed to behave in this region. 


\subsubsection{Other Fragmentation Functions}

A number of other fragmentation functions have been proposed. The different functions will be tested in chapter 7 by replacing the Lund Symmetric Fragmentation Function inside the Pythia Monte Carlo generator.

The Peterson function [7] is often used instead of the Lund Symmetric Fragmentation Function to describe the fragmentation of heavy $\mathrm{c}$ and b quarks. It comes about by simply taking the amplitude for the fragmentation transition to be proportional to $\frac{1}{\Delta E}$.

The transition from a heavy quark of mass $M_{Q}$ and momentum $P$, to a hadron of mass $M_{H}$ and momentum $z P$, plus a light quark of mass $M_{q}$ and momentum $(1-z) P$ is

$$
\Delta E=\sqrt{M_{Q}^{2}+P^{2}}-\sqrt{M_{H}^{2}+z^{2} P^{2}}-\sqrt{M_{q}^{2}+(1-z)^{2} P^{2}}
$$

Now, assuming that $M_{Q}=M_{H} \ll z P$ and including a $\frac{1}{z}$ phase space term, we get the Peterson fragmentation function

$$
f(z)=\frac{1}{z}\left(1-\frac{1}{z}-\frac{\epsilon}{1-z}\right)^{-2}
$$

where $\epsilon$ is predicted to be $\left(M_{q} / M_{Q}\right)^{2}$, but in practice is fit to the data. It should be noted that the first assumption $\left(M_{Q}=M_{H}\right)$ is not true for the $\Lambda_{c}$, which has a mass of $2.285 \mathrm{GeV}$ compared to a mass of $\sim 1.1 \mathrm{GeV}$ for the charm quark, and the second assumption $\left(M_{H} \ll z P\right)$ is not true at BaBar energies. It turns out that most of the available fragmentation functions were designed specifically for heavy meson production, as baryon formation is not well understood theoretically. Therefore, the results of this paper should be thought of as a target for future models of baryon production, and not simply as a test to see which heavy quark meson fragmentation function happens to fit the data best. 
A desirable feature for a fragmentation function is that it match the hadron structure function in the limit of high $\mathrm{z}$. This means that the probablity of a quark fragmenting into a hadron with just slightly less momentum should be equal to the probability, in a deep inelastic scattering experiment, of finding a quark that contains almost all of the momentum of the parent hadron.

$$
f(z) \rightarrow(1-z)^{2 N_{s}-1} \quad \text { as } \quad z \rightarrow 1
$$

where $N_{s}$ is the number of spectator quarks. We expect the exponent to be 1 for mesons and 3 for baryons, although some theorists believe that an additional $(1-z)$ suppresion factor might be needed when the fragmentation flips the helicity [8]. Expanding the Peterson function around $z=1$ shows that it has an exponent of two. The Kartvelishvili fragmentation function [9]

$$
f(z)=z^{\alpha_{b}}(1-z)
$$

fixes the exponent of $(1-z)$ to one, and fits the exponent of $z$ to the data. Again this model is specifically designed for fragmentation into mesons. We will also test a modified Kartvelishvili fragmentation function

$$
f(z)=z^{\alpha_{b}}(1-z)^{3}
$$

to see if we get a better fit to the $\Lambda_{c}$ data.

In the next section, we will see that the UCLA fragmentation model [14] is based on the idea that the probability of a particular event occuring is related to the space time area swept out by the string. For massless quarks, the area of one period of a yo-yo meson is equal to one half of the mass of the meson squared. For heavy quarks, which follow hyperbolic paths, this formula is altered. The Bowler fragmentation function [10] 


$$
f(z)=\frac{1}{z^{\left(1+b m_{\perp}^{2}\right)}}(1-z)^{a} \exp \left(-b m_{\perp}^{2} / z\right)
$$

modifies the Lund function by taking this change in area into account. The result is a softening of the spectra of heavy quark hadrons.

Two more fragmentation functions are based on perturbative QCD. The Collins and Spiller function [11]

$$
f(z)=\left(\frac{1-z}{z}+\frac{(2-z) \epsilon_{b}}{1-z}\right)\left(1+z^{2}\right)\left(1-\frac{1}{z}-\frac{\epsilon_{b}}{1-z}\right)^{-2}
$$

is a first order calculation which, like the Kartvelishvili function, requires a $(1-z)$ behavior as $z \rightarrow 1$.

The function proposed by Braaten, Cheung, Fleming and Yuan (BCFY)[12] is calculated to next-to-leading order in the heavy-quark mass expansion.

$$
\begin{gathered}
f(z)=\frac{z(1-z)^{2}}{[1-(1-r) z]^{6}}\left[3+\sum_{i=1}^{4}(-z)^{i} f_{i}(r)\right] \\
f_{1}(r)=3(3-4 r) \\
f_{2}(r)=12-23 r+26 r^{2} \\
f_{3}(r)=(1-r)\left(9-11 r+12 r^{2}\right) \\
f_{4}(r)=3(1-r)^{2}\left(1-r+r^{2}\right)
\end{gathered}
$$

It is not clear how this function or the one by Collins and Spiller should be modified to model the $\Lambda_{c}$ spectrum, so they will be tested as is.

We will also be testing the HERWIG [13] model, which is completely independant from the JETSET/Pythia algorithm. At the end of the parton shower, the HERWIG model groups the partons into color singlet clusters, which then decay by phase space into two hadrons. The suppressed rates for baryon production are controlled by this phase space decay. 


\subsubsection{The UCLA Model}

If one looks at the main suppression factors in the Lund model - strange quark suppression, vector suppression and, as will be seen in the next section, baryon suppression - one is struck by the fact that all of these factors are doing essentially the same thing: namely, suppressing the creation of higher mass particles. The UCLA fragmentation model tries to find a common origin, related to the final hadron mass, for all of the various suppression factors in the Lund model.

Going back to the Lund Symmetric Fragmentation Function (2.7), we see that mass suppression is already built into the formula. In the Lund algorithm, however, the fragmentation function is only used to find the particle momentum. The mass of the particle has already been determined beforehand and the suppression of high mass particles is achieved by the various suppression factors (equations 2.5 and 2.6). The UCLA model combines mass suppression and momentum determination into a single equation

$$
f\left(z, m_{h}\right)=N C^{2} \frac{(1-z)^{a}}{z}\left(1-\frac{m_{h}^{2}}{S z}\right)^{a} e^{-b m_{h} / z_{e f f}}
$$

where $\mathrm{N}$ is a normalization constant, $\mathrm{C}$ is a Clebsch-Gordan coefficient for both spin and flavor, $m_{h}$ is the mass of the created hadron, $\mathrm{S}$ is the squared energy of the string system that fragmented to create the hadron, and $z_{\text {eff }}$ is the effective $\mathrm{z}$, after correcting for the difference in the area swept out by non-ultrarelativistic heavy quarks. Now $\mathrm{f}$ is normalized such that

$$
\int \sum_{\text {known hadrons }} f\left(z, m_{h}\right) d z=1
$$

and thus it gives the probability of finding a particular type of hadron at a particular momentum.

For large $\mathrm{S}$ and small quark mass, the shape of the UCLA fragmentation 
function is identical to the Lund function. The difference comes from the fact that the UCLA model takes the space-time area law literally. That is, the probability of any event occuring is proportional to $e^{-b A}$, where $\mathrm{A}$ is the total space-time area swept out by the event.

This idea has its origin in lattice QCD calculations. Lattice QCD is beyond the scope of this paper, but the basic idea is to discretize equation (2.3) and then to look for quark paths that minimize the action. It turns out that the gluonic action dominates. Looking at figure 2.1 one sees that during the hadronization stage gluons will be present in the shaded region, and it is this region which defines the space time area $\mathrm{A}$ in the following formulas. The coefficient "b" is related to the coupling strength. In addition, the fermionic action gives rise to a suppression of events with longer quark path lengths. The sum of the quark path lengths is equal to the perimeter of the shaded region, and so the fermionic action term gives a suppression related to the perimeter of the event. This term is ignored in meson production, but will be seen again in the section on popcorn mesons between baryons.

Armed with the area law, we can write down the probability of $\mathrm{N}$ hadrons forming with energies $E_{i}$, and momenta $P_{i}$ as being

$$
d \mathcal{P}\left(E_{1}, P_{1}, \ldots E_{n}, P_{n}\right)=\frac{e^{-b A}}{g(s)} \delta\left(\sqrt{s}-\sum_{1}^{N} E_{i}\right) \delta\left(\sum_{1}^{N} P_{i}\right) \delta\left(E_{i}^{2}-P_{i}^{2}-m_{i}^{2}\right) d E_{i} d P_{i}
$$

where $e^{-b A}$ is the area law suppression factor and $\mathrm{g}(\mathrm{s})$ is a normalization factor such that the sum of all probabilities for a particular center of mass energy squared $\mathrm{s}$ is equal to one. Changing to the light cone variable $\mathrm{z}$ gives

$$
d \mathcal{P}\left(z_{1}, z_{2} \ldots z_{n}\right)=\frac{e^{-b A}}{g(s)} \sum_{i=1}^{N} \frac{1}{z_{i}} \delta\left(s-\sum_{1}^{N} \frac{m_{i}^{2}}{z_{i}}\right) \delta\left(1-\sum_{1}^{N} z_{i}\right) d z_{i}
$$

Using this expression we can derive a fragmentation function by dividing the 


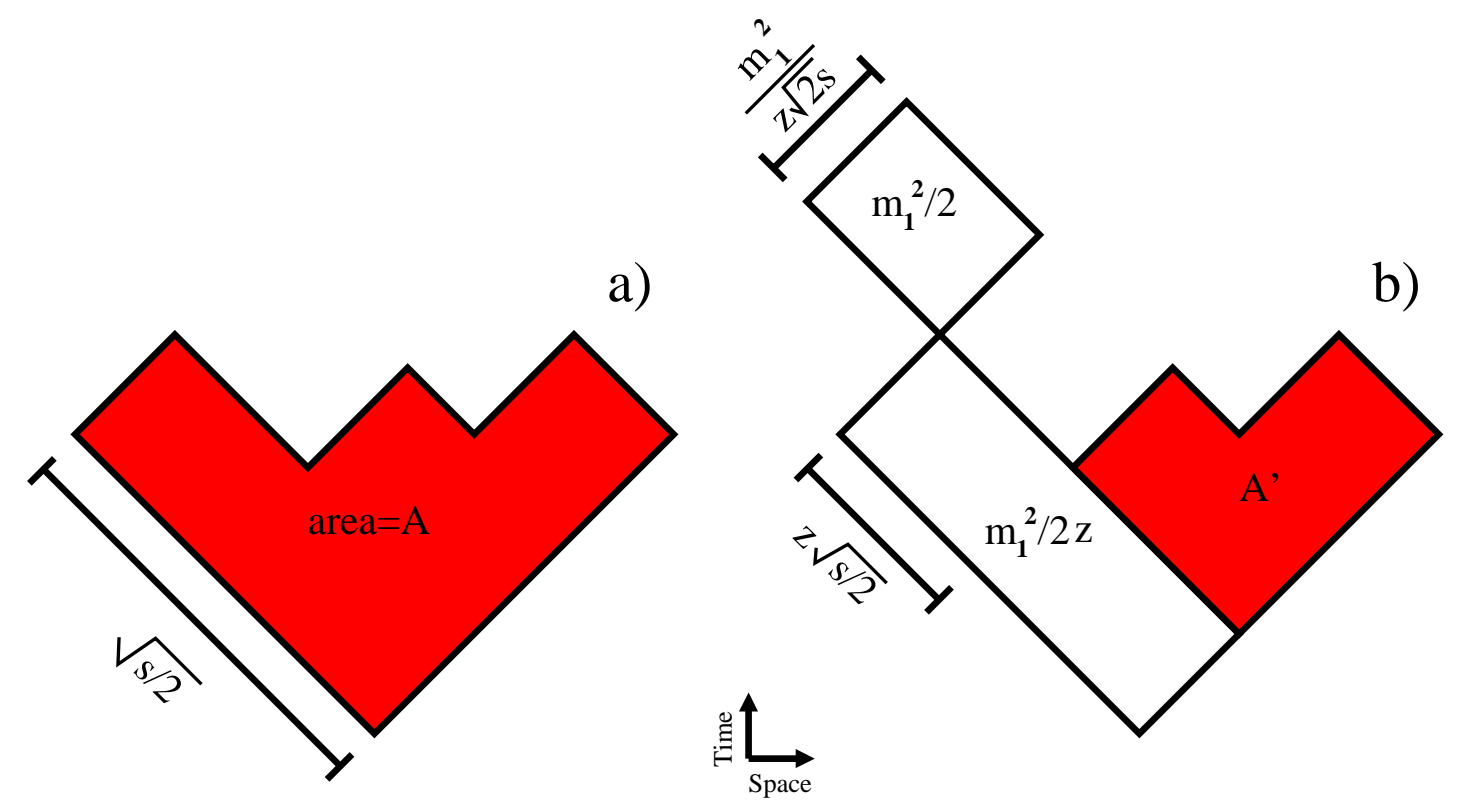

Figure 2.2: a) the total space-time area swept out by a string fragmenting into 3 mesons. b) the remaining area A' after the creation of a meson of mass $m_{1}$.

area $\mathrm{A}$, shown in figure 2.2 , into the parts used up by the creation of the outermost hadron, $m_{1}^{2} / 2 z$, and the remaining area $A^{\prime}$. The conversion from area to mass is done by noticing that, when the yo-yo meson quarks reach their turn around point, all of the energy is stored in the string, which has an energy density $\mathrm{k}$ of about $1 \mathrm{GeV} /$ fermi. The momentum of a particle is the time difference of the two widest points, times k. From this, we calculate z using the energy-momentum relation, resulting in the values in the figure.

$d \mathcal{P}$ now divides neatly into two parts

$$
d \mathcal{P}\left(z_{1}, z_{2} \ldots z_{n}\right)=\left[\frac{e^{-\frac{b m_{1}^{2}}{2 z}}}{g(s)} \frac{d z}{z_{1}}\right]\left[g\left(s^{\prime}\right) d \mathcal{P}^{\prime}\left(z_{2} \ldots z_{n}\right)\right]
$$

where 


$$
d P^{\prime}\left(z_{2} \ldots z_{n}\right)=\frac{e^{-b A^{\prime}}}{g\left(s^{\prime}\right)} \sum_{i=2}^{N} \frac{1}{z_{i}} \delta\left(s-\frac{m_{1}^{2}}{z_{1}}-\sum_{2}^{N} \frac{m_{i}^{2}}{z_{i}}\right) \delta\left(1-z_{1}-\sum_{2}^{N} z_{i}\right) d z_{2} \ldots d z_{N}
$$

Equation (2.11) is equivalent to (2.9) with the energy and momentum of the first particle removed. We can now calculate the probability of a particular first fragmentation by integrating (2.10) over all the other $z_{i}$. This is easy since the integral of $d \mathcal{P}^{\prime}$ is defined to be one. So we have the following fragmentation function

$$
f\left(z, m_{h}\right)=\frac{g\left(s^{\prime}\right)}{g(s)} e^{-\frac{b m_{h}^{2}}{2 z}}
$$

Now, if the fragmentation function changes slowly compared to the number of possible states at a given s,

$$
\frac{d f / d s}{f} \ll \frac{d g / d s}{g}
$$

it can be shown [14] that

$$
\frac{g\left(s^{\prime}\right)}{g(s)}=\left(\frac{s^{\prime}}{s}\right)^{a}
$$

Remembering that $\mathrm{s}$ is just twice the area of the yo-yo meson that would form if no fragmentation occurred, and reading these areas off of figure 2.2, we get the final form of the UCLA fragmentation function

$$
f\left(z, m_{h}\right)=\frac{(1-z)^{a}}{z}\left(1-\frac{m_{h}^{2}}{S z}\right)^{a} e^{-b m_{h}^{2} / z}
$$

The coefficient $\mathrm{b}$ has been redefined to get rid of the $1 / 2$. Actually, it goes away naturally if the space-time area is converted to light cone variable area as in the original paper.

Once the UCLA fragmentation function is derived, it can be used to generate Monte Carlo events by putting it inside a modified version of the JETSET code. 
As a result, the UCLA model uses the same parton shower code and "outside-in" iterative procedure for choosing hadrons.

The effectiveness of the space-time area law as a way of chosing particle flavor can be seen in Tables 2.4 and 2.5. The UCLA model is able to produce results similar to JETSET's, while utilzing only six free parameters, compared to about 17 for JETSET. Readers interested in the $\Lambda_{c}$ spectrum predictions can look ahead to figure 7.4. Light and heavy meson spectra predictions can be seen in [14] and [15], respectively.

\subsection{Baryon Formation}

In order to produce the mesons described in the previous section, we needed to combine a quark and antiquark in such a way that the colors formed a singlet state. It was obvious that the way to do this was match a red quark with an antired antiquark, a blue with an antiblue and a green with an antigreen. In order to make a baryon, we need to form color singlet states of three quarks, out of a series of quark-antiquark color singlet pairs. It is not obvious how this will work, and so we will need a system to keep track of how color is flowing between each quark.

Lets look at meson formation again in terms of group theory. The original quark-antiquark pair coming from the virtual photon will be in a singlet state $\frac{1}{\sqrt{3}}(r \bar{r}+b \bar{b}+g \bar{g})$. Now a new quark-antiquark pair tunnels out of the string. Because of the symmetry of the original pair, the relationship between the old and new quarks on the right side, will be the same as that between the old and new quarks on the left side. Thus, it is sufficient to look at what happens on one side only. In order to form a meson we need a quark and an antiquark so we have 


$$
3 \otimes \overline{3}=8 \oplus 1
$$

If the tunneling quarks form an octet configuration with the original quarks, a repulsive force will result and the still virtual new quarks will be pushed back and eventually recombine. If a singlet state is formed, we now have, on each side, a system that looks exactly like the original quark-string-antiquark. These will either form yo-yo mesons or divide again as described in the previous section.

If a quark tunnels out on the side of the original quark, we have

$$
3 \otimes 3=\overline{3} \oplus 6
$$

The sextet state is repulsive and, like the octet state will quickly decay. The anti-triplet state is attractive, but, because only color singlet states can have an independant existence, it will still send a flux of color to the other side of the event. The interesting thing to notice is that the quark $\otimes$ quark anti-triplet state has the same color as an antiquark state. Thus, from the point of view of the central region of the string, it looks as if the endpoints are still a quark-antiquark pair in a color singlet state.

The flow of color between quarks is much easier to follow when represented graphically. Casher et al. came up with a system for doing this in the first paper written on popcorn mesons [16]. In figure 2.3 quarks are labelled R, B and G and the color flows $\lambda_{3}$ and $\lambda_{8}$, corresponding to the diagonal Gell-Mann matricies. Of course, the actual quark states will be superpositions of R, B, and G and the other 6 gluons will contribute to the forces, but it is easy to verify that this graphical system corresponds to the properties derived from group theory. The antiquark in a singlet state absorbs exactly the same colors as those emitted from the corresponding quark, and the flux coming from two quarks of different colors is 


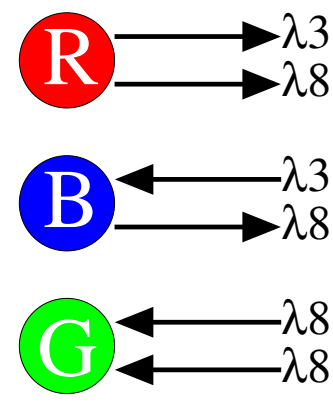

Quarks
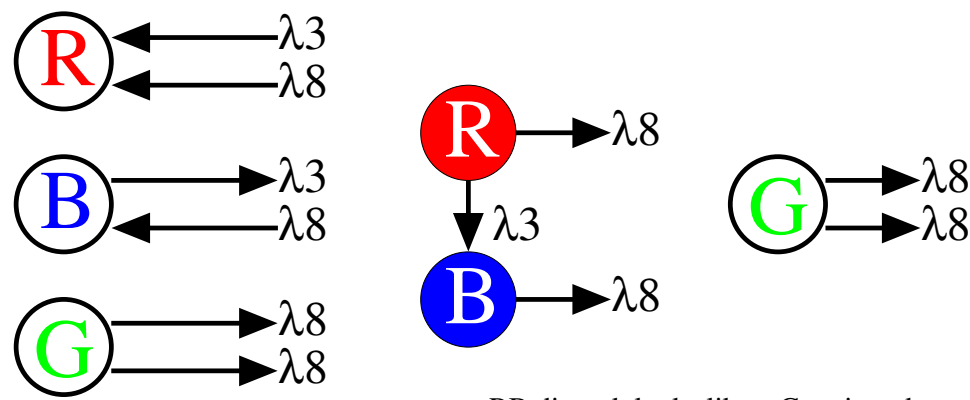

$\mathrm{B} \rightarrow \lambda 8$

a RB diquark looks like a $\mathrm{G}$ antiquark

Figure 2.3: Color flows from quarks, antiquarks and diquarks

the same as that from the antiquark of the third color. Because the quark $\otimes$ quark anti-triplet state is attractive, it is sometimes thought of as a single object known as a diquark.

These diagrams can also be thought of as the first term of a symmetrized wave function. For example, the $r \bar{r}$ color diagram in figure $2.4 \mathrm{a}$ corresponds to the wavefunction $\frac{1}{\sqrt{3}}(r \bar{r}+b \bar{b}+g \bar{g})$ and the $r b g \bar{g} \bar{b} \bar{r}$ diagram in figure 2.5 corresponds to

$$
\frac{1}{\sqrt{6}}(r b g \bar{g} \bar{b} \bar{r}+b g r \bar{r} \bar{g} \bar{b}+g r b \bar{b} \bar{r} \bar{g}-r g b \bar{b} \bar{g} \bar{r}-g b r \bar{r} \bar{b} \bar{g}-\operatorname{brg} \bar{g} \bar{r} \bar{b})
$$

Let's use these color flow diagrams to study baryon formation. Figure 2.4 shows a $e^{+} e^{-} \rightarrow 2$ baryons in four steps. First the $e^{+} e^{-}$annihilate, giving rise to a red-antired pair. Then, a blue-antiblue pair tunnel out of the string. We know that the color flux from a red-blue pair is the same as that from an antigreen antiquark. Thus the field in the middle of the event looks like it is created by an antigreen-green pair. The energy density between an antigreen-green pair is the same as that between a red-antired pair, the type of field that we see on the two outer sections of the string. This means that if we were to move the blue quarks to the right or to the left, we would not change the energy of the system. 


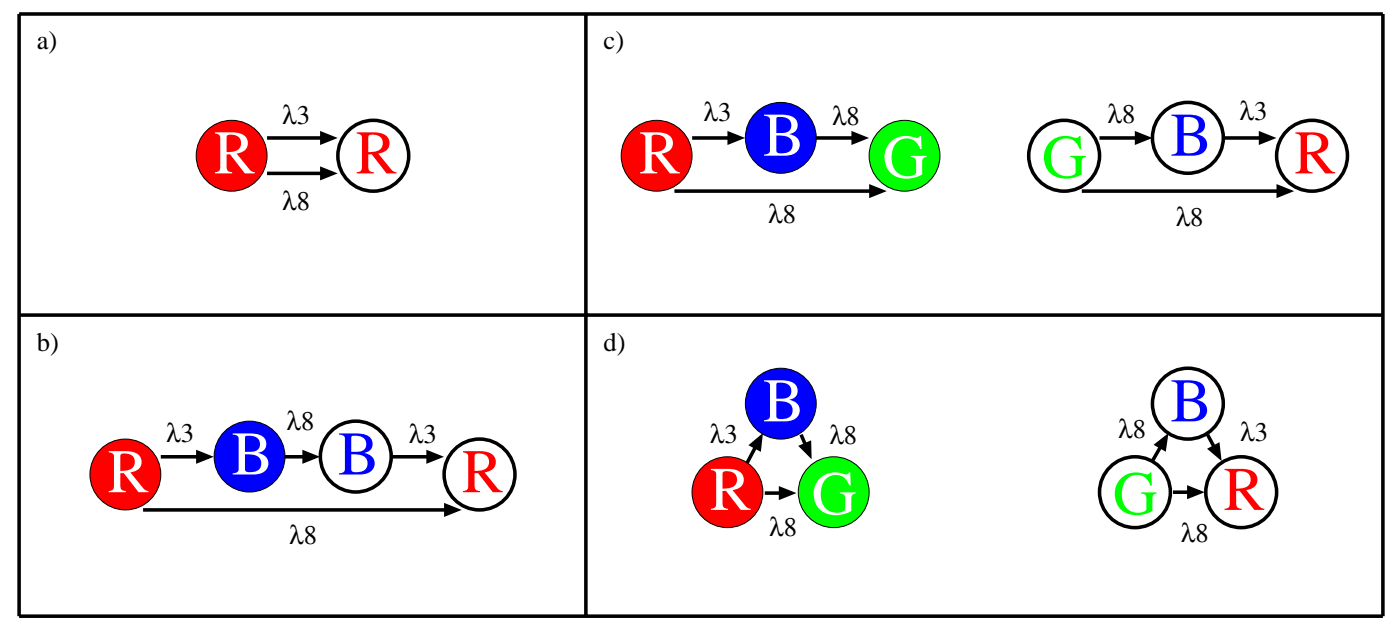

Figure 2.4: Baryon formation from a string. a) a virtual photon decays into $r \bar{r}$. b) a $b \bar{b}$ pair tunnels from the string. c) creation of a $g \bar{g}$ pair blocks the flow of color from one side of the event to the other. d) the quarks rearrange themselves in two dimensions forming a baryon-antibaryon pair.

Therefore, there is no net force on the blue quarks.

In the third step, a green-antigreen pair is created. These quarks absorb the two $\Lambda_{8}$ color fluxes in the middle of the event, splitting the string into two pieces in the same way that was seen before in meson formation. We now have a baryon-antibaryon pair.

\subsubsection{Baryons in the Lund Model}

In the Lund model, the probability of a baryon forming can be estimated using the WKB formula that we used for meson formation. The result is

$$
P_{\text {baryon }}=\exp \left(-\frac{4 m_{b}\left(m_{b}+m_{g}\right)+\pi m_{g}\left(m_{b}+m_{g}\right)}{k}\right)
$$

where $m_{b}$ and $m_{g}$ are the masses of the blue and green quarks in figure 2.4. The coefficient in front of the blue quark is 4 and not $\pi$, as in the case of a meson, 
because the blue quark floats freely between two pieces of string and thus its creation is more heavily suppressed than that of the green quark, which is pulled in the direction of the baryon. The $\left(m_{b}+m_{g}\right) / k$ term is the distance that the blue and green quarks need to tunnel in order to come onto the mass shell, and is proportial to the energy of the portion of string eaten up in order to create the new quark masses.

One often thinks of the green and blue quarks forming a diquark of mass $\mu$. In this case the baryon formation probability is identical to that of meson formation (2.5), with the diquark mass replacing the mass of the second quark in the meson.

$$
P_{\text {baryon }}=\exp \left(-\frac{\pi \mu^{2}}{k}\right)
$$

If we think of the 4 and $\pi$ in (2.12) as being approximately equal, we get an estimated diquark mass of

$$
\mu \sim\left(m_{b}+m_{g}\right)
$$

which is not too unreasonable, although in practice the diquark masses are chosen such that baryon formation occurs at about $\frac{1}{10}$ of the rate of meson formation.

The process shown in figure 2.4 can be continued one step further to create a baryon - popcorn meson - antibaryon event as shown in figure 2.5. Now the blue quarks have to tunnel a long additional distance, $k \sqrt{M}$, inside the string in order to make room for the meson being made from the energy density in the middle. An additional suppresion factor of

$$
P_{\text {popcorn }}=\exp \left(-\frac{2 m_{b} M}{k}\right)
$$

is multiplied to (2.12) in order to get the suppression of popcorn baryon systems. Here the $\mathrm{M}$ can stand for the mass of a single meson, or a system of several mesons. In practice, the popcorn suppresion factor is tuned to the data like the other parameters. The current Lund algorithm creates baryon-antibaryon pairs 

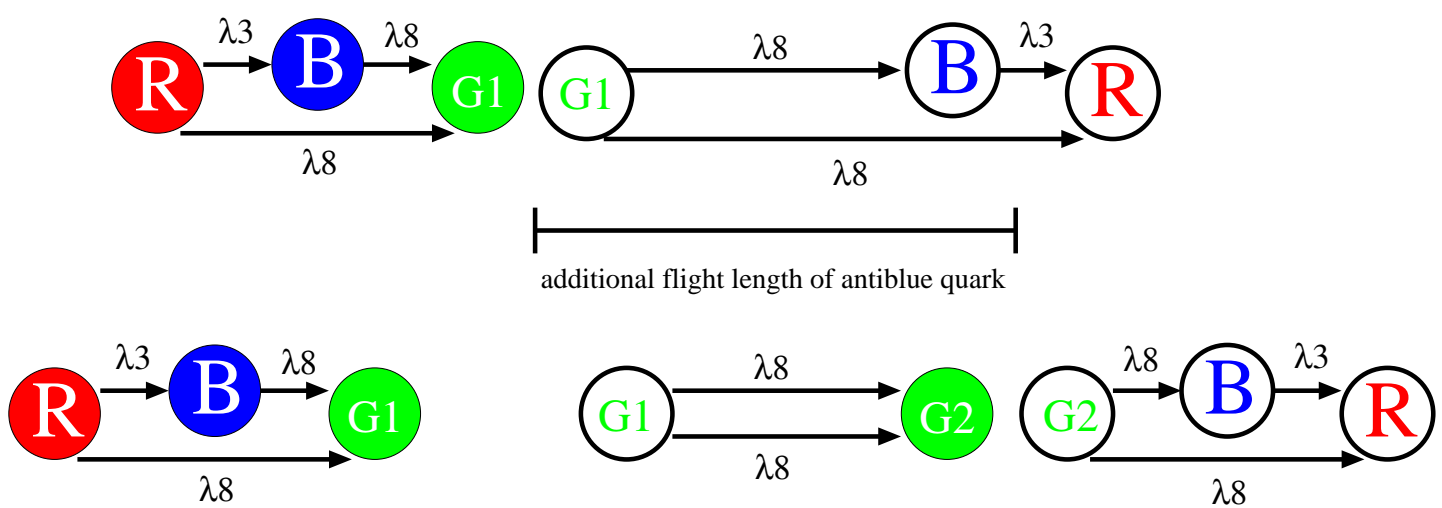

Figure 2.5: Formation of a popcorn meson. Suppression of these events is caused by the additional flight length of the antiblue quark compared to figure 2.4.

with no popcorn mesons $50 \%$ of the time and one popcorn meson the other $50 \%$ of the time.

It should be noted that if the $\bar{b}$ and $\bar{g} 1$ quarks in figure 2.5 were always bound together into a diquark, the second green quark pair could not appear, and the baryons would always be created adjacent to each other. At one time it was believed that baryons were always created from diquarks, but the experimental results to be summarized in section 2.3.3 seem to contradict this idea.

\subsubsection{Baryons in the UCLA model}

Unlike the Lund model, the UCLA model allows arbitrarily long popcorn meson chains between a baryon-antibaryon pair. Figure 2.6 shows the topologies of the possible space-time area diagrams for zero, one, two and three popcorn mesons. For simplicity, both quarks and anti-quarks are drawn in the same color. The number of different color orderings for a particular number of popcorn mesons increases rapidly with the number of popcorn mesons. We see in figure 2.6 that there are already four ways of creating three popcorn mesons. Adding together 
the area law predictions for all of these possibilities leads to an overprediction of the number of baryons. However, looking back at section 2.2.3, we see that we have left out a perimeter suppression term, which would act to suppress these long popcorn chains. Looking at figure 2.6 we see an extra virtual quark flight length between the two baryons when a popcorn meson is present. Sometimes this flight length is shared by two or more quarks, but it is always equal to $\sqrt{2}$ times the distance between the break points which separate the baryons from the rest of the string. The mass of the popcorn system $M$ is just this distance times the energy density $\mathrm{k}$, so we end up with a suppression factor depending on the popcorn mass

$$
P_{\text {popcorn }}=\exp (-\eta M)
$$

where $\eta$ has been tuned to be $3.5 \mathrm{GeV} / \mathrm{c}$. The form of this equation is identical to that of the Lund model (2.13), but the UCLA model differs in that it allows arbitrary popcorn configurations instead of limiting the number of popcorn to zero or one.

In chapter 8 we will be looking at $10.58 \mathrm{GeV}$ events with two $\Lambda_{c}$. Because c quarks are too heavy to be created from the string, we will know that the $\Lambda_{c}$ baryons are on the outside of the event, unless they decayed from a heavier charmed baryon. Thus, any mesons in the event, other than those from decays of charmed baryons, will be popcorn mesons.

Unfortunately, because the Monte Carlo algorithm for matching up the two sides of the event requires baryon conservation on each side, we are not able to use the standard UCLA generator for predicting the amount of popcorn. Figure 2.7 gives an estimate of the expected amount of popcorn using the area and perimeter suppression factors. The red curve shows the amount of mass left over after applying the UCLA fragmentation function twice to create the two $\Lambda_{c}$. This 


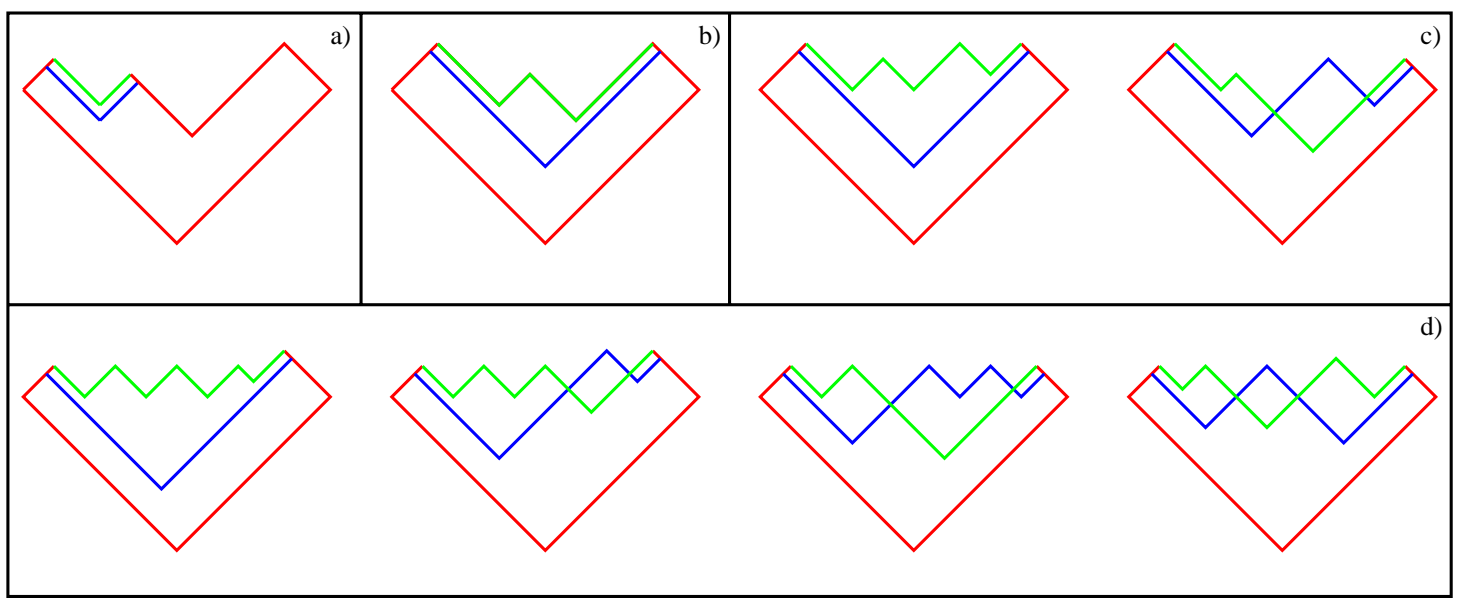

Figure 2.6: Space-time area diagrams for a) zero, b) one, c) two, and d) three popcorn mesons.

procedure ignores initial hard gluon radiation, and the transverse momenta of the $\Lambda_{c}$, but it still should provide a reasonable estimate of what we would expect if the $\Lambda_{c}$ particle were a meson, and the extra perimeter suppression were not applicable. The black curve shows the expected popcorn system mass with the perimeter suppression. There are still lots of entries with more than a $1 \mathrm{GeV}$ mass, so the UCLA model seems to be suggesting lots of events with several popcorn mesons.

\subsubsection{Previous Evidence for Popcorn}

This section will give a brief summary of some previous experimental searches for popcorn mesons.

According to the string model, if some kind of popcorn mechanism is not present, then baryon-antibaryon pairs will always be produced adjacent to each other and will always share at least two quarks. Since strange quarks are produced 


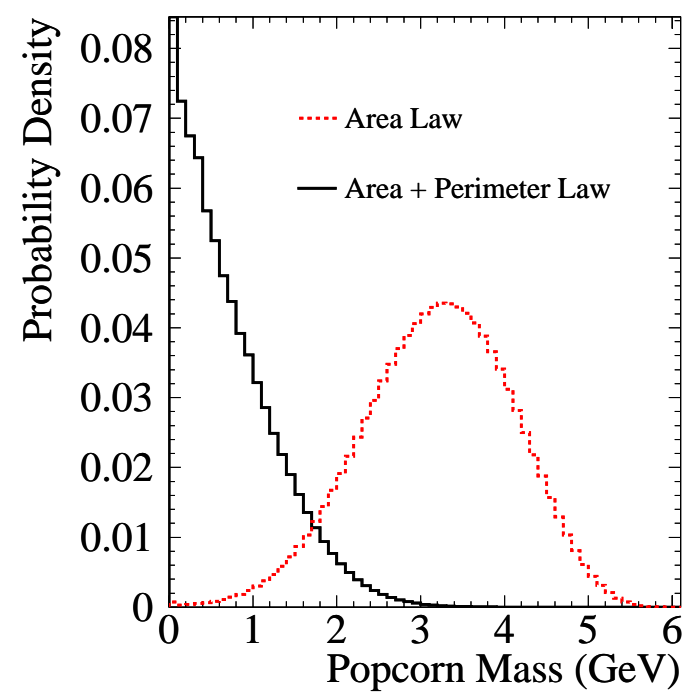

Figure 2.7: UCLA model estimate of the mass in between two leading $\Lambda_{c}$, with and without suppression of virtual quarks with long flight lengths

at a relatively low rate, they can be used as a marker for quark sharing between baryons.

The OPAL experiment at LEP measured the rates of events with an identified strange baryon and a strange antibaryon [17]. The results are shown in table 2.2. With no popcorn, the $\Xi^{-}$will possess either a $d s$ or an $s s$ diquark meaning that a strange antibaryon must be present in the event. Comparing the data to the Monte Carlo with no popcorn, we see that the strange antibaryon is found less often than predicted. If popcorn mesons are included in the Monte Carlo, the predicted rate of the strange antibaryon drops because the mesons can be taking away one or both of the strange antiquarks, leaving a non-strange anti-baryon on the other side. Including a popcorn meson between $95 \%$ of the baryon-antibaryon pairs fits the OPAL data better, but the result is not very significant statistically. 


\begin{tabular}{|c|c|c|c|c|}
\hline & & \multicolumn{3}{|c|}{ probability } \\
\hline tagging & tagged & OPAL & JETSET & JETSET \\
\hline baryon & baryon & data & no popcorn & $95 \%$ popcorn \\
\hline$\Xi^{-}(d s s)$ & $\bar{\Lambda}(\bar{u} \bar{d} \bar{s})$ & $.463 \pm .099$ & .589 & .412 \\
\hline$\Xi^{-}(d s s)$ & $\bar{\Xi}^{+}(\bar{d} \bar{s} \bar{s})$ & $.037 \pm .065$ & .172 & .071 \\
\hline
\end{tabular}

Table 2.2: Probability of finding a strange antibaryon in events that already have a strange baryon.

Another way of looking for popcorn mesons is to measure the rapidity correlations between strange baryons. Because the UCLA and Lund fragmentation functions tend to give particle distributions that are flat in rapidity space, the inclusion of a popcorn meson between two baryons should increase their rapidity difference. This analysis was done by the DELPHI collaboration [18]. Figure 2.8 shows the rapidity difference between $\Lambda$ and $\bar{\Lambda}$ in $Z^{0}$ decays. The best fitting Monte Carlo seems to be the one that includes a popcorn meson $50 \%$ of the time.

A third expected consequence of the popcorn model is the observation of strange mesons with rapidities falling between the rapidities of an observed $\Lambda \bar{p}$ pair. The strange popcorn meson contains the anti-strange partner of the $s$ quark in the $\Lambda$ which is not compensated for in the proton. The results of this Delphi measurement [19] are shown in table 2.3.

Only closely correlated baron pairs with a rapidity difference of less than one unit are considered. The data do not show an increase in kaons compared to the $p \bar{p}$ and $\Lambda \bar{\Lambda}$ control samples. This is because the decay of unstable particles smear the correspondance between rapidity and the rank in the string. The JETSET 7.3 Monte Carlo, with a 50\% popcorn probability confirms that the result is consistant with the model. This experiment shows us that it is very 


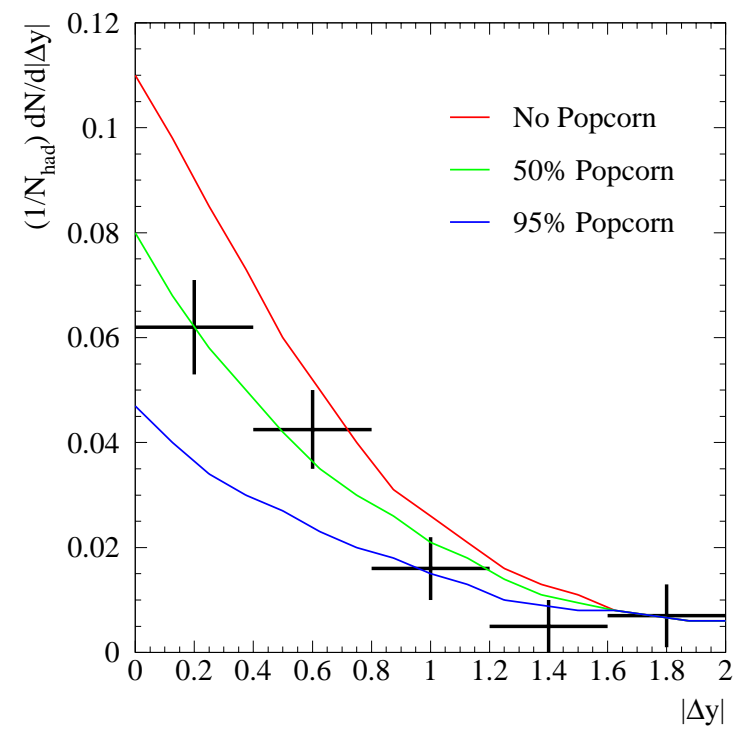

Figure 2.8: Rapidity difference between $\Lambda$ and $\bar{\Lambda}$ in $Z^{0}$ decays.

difficult to directly observe popcorn mesons between closely correlated baryon pairs. Our search at BaBar, described in chapter 8, is different because we know that the charmed baryons only appear on the ends of the string, and thus have a large rapidity difference. Decays of the known heavy charmed baryons are expected to be symmetric around the daughter $\Lambda_{c}$ particle and so they should be distinguishable from popcorn mesons, which will tend to appear in the rapidity range between the $\Lambda_{c} \bar{\Lambda}_{c}$ pair. If large numbers of popcorn mesons exist in $\Lambda_{c} \bar{\Lambda}_{c}$ events, they should be easy to observe at BaBar. 


\begin{tabular}{|c|c|c|c|c|}
\hline & \multicolumn{2}{|c|}{ Data } & \multicolumn{2}{c|}{ Monte Carlo } \\
\hline$B \bar{B}$ pair & fraction of $B \bar{B}$ pairs with & \multicolumn{2}{c|}{ fraction of $B \bar{B}$ pairs with } \\
\hline & one pion & one kaon & one pion & one kaon \\
\hline$p \bar{p}$ & $0.25 \pm 0.02$ & $0.07 \pm 0.01$ & $0.25 \pm 0.01$ & $0.07 \pm 0.01$ \\
$\Lambda \bar{\Lambda}$ & $0.26 \pm 0.03$ & $0.07 \pm 0.01$ & $0.24 \pm 0.01$ & $0.07 \pm 0.01$ \\
$\Lambda \bar{p}$ & $0.26 \pm 0.02$ & $0.05 \pm 0.01$ & $0.25 \pm 0.01$ & $0.06 \pm 0.01$ \\
\hline
\end{tabular}

Table 2.3: Fractions of $B \bar{B}$ pairs with kaons and pions in the rapidity gap between the two baryons. 


\begin{tabular}{|l|c|r|r|l|l|l|}
\hline Particle & Quarks & Spin & Mass & Multiplicity & Lund & UCLA \\
\hline$\pi^{+}$ & $u \bar{d}$ & 0 & .140 & $6.6 \pm 0.2$ & 6.47 & 6.39 \\
$\pi^{0}$ & $(u \bar{u}-d \bar{d}) / \sqrt{2}$ & 0 & .135 & $3.2 \pm 0.3$ & 3.69 & 3.68 \\
$K^{+}$ & $u \bar{s}$ & 0 & .494 & $0.90 \pm 0.04$ & 1.02 & 1.04 \\
$K^{0}$ & $d \bar{s}$ & 0 & .498 & $0.91 \pm 0.05$ & 0.847 & 0.916 \\
$\eta$ & $(u \bar{u}+d \bar{d}-2 s \bar{s}) / \sqrt{6}$ & 0 & .549 & $0.20 \pm 0.04$ & 0.404 & 0.281 \\
$\eta^{\prime}$ & $(u \bar{u}+d \bar{d}+s \bar{s}) / \sqrt{3}$ & 0 & .958 & $0.03 \pm 0.01$ & 0.088 & 0.051 \\
$D^{+}$ & $c \bar{d}$ & 0 & 1.869 & $0.16 \pm 0.03$ & 0.178 & 0.210 \\
$D^{0}$ & $c \bar{u}$ & 0 & 1.865 & $0.37 \pm 0.06$ & 0.473 & 0.473 \\
$D^{S}$ & $c \bar{s}$ & 0 & 1.971 & $0.13 \pm 0.02$ & 0.085 & 0.085 \\
$\rho^{0}$ & $(u \bar{u}-d \bar{d}) / \sqrt{2}$ & 1 & .770 & $0.35 \pm 0.04$ & 0.457 & 0.422 \\
$\omega$ & $(u \bar{u}+d \bar{d}) / \sqrt{2}$ & 1 & .783 & $0.30 \pm 0.08$ & 0.403 & 0.336 \\
$K^{*+}$ & $u \bar{s}$ & 1 & .892 & $0.27 \pm 0.03$ & 0.387 & 0.349 \\
$K^{* 0}$ & $c \bar{s}$ & 1 & .904 & $0.29 \pm 0.03$ & 0.332 & 0.321 \\
$\phi$ & $s \bar{s}$ & 1 & 1.020 & $0.044 \pm 0.003$ & 0.063 & 0.062 \\
$D^{*+}$ & $c \bar{d}$ & 2.010 & $0.22 \pm 0.04$ & 0.228 & 0.197 \\
$D^{* 0}$ & $x .007$ & $0.23 \pm 0.06$ & 0.228 & 0.200 \\
\hline
\end{tabular}

Table 2.4: Meson Multiplicities at 10.58 GeV. PDG vs. Monte Carlo. 


\begin{tabular}{|l|c|c|c|l|l|c|}
\hline Particle & Quarks & Spin & Mass & Multiplicity & Lund & UCLA \\
\hline$p$ & uud & $\frac{1}{2}$ & 0.938 & $.253 \pm .016$ & .360 & .221 \\
$\Lambda$ & $u d s$ & $\frac{1}{2}$ & 1.116 & $.080 \pm .007$ & .112 & .092 \\
$\Sigma^{0}$ & $u d s$ & $\frac{1}{2}$ & 1.193 & $.023 \pm .008$ & .0223 & .0223 \\
$\Xi$ & $d s s$ & $\frac{1}{2}$ & 1.321 & $.0059 \pm .0007$ & .0079 & .0054 \\
$\Lambda_{c}$ & $u d c$ & $\frac{1}{2}$ & 2.285 & $.100 \pm .030$ & .054 & .0244 \\
$\Delta^{++}$ & uuu & $\frac{3}{2}$ & 1.197 & $.040 \pm .010$ & .053 & .022 \\
$\Xi^{* 0}$ & uss & $\frac{3}{2}$ & 1.526 & $.0015 \pm .0006$ & .00136 & .00168 \\
$\Omega^{-}$ & sss & $\frac{3}{2}$ & 1.672 & $.0007 \pm .0004$ & .00017 & .00017 \\
\hline
\end{tabular}

Table 2.5: Baryon Multiplicities at $10.58 \mathrm{GeV}$. PDG vs. Monte Carlo 


\section{CHAPTER 3}

\section{The BaBar Detector}

The BaBar detector is described in great detail elsewhere [20]. This chapter will only give a short description of each of the parts.

\subsection{Detector Asymmetry}

BaBar detects particles created in collisions between $9 \mathrm{GeV}$ electrons and $3.1 \mathrm{GeV}$

positrons, which corresponds to a boosted center of mass frame with a $\beta \gamma$ of 0.56 . The experiment is designed asymmetrically in order to optimize the measurement of the B meson lifetime differences, which are of the order of picoseconds. The lifetimes are measured by the flight lengths of the B mesons in the direction of the boosted center of mass frame. This boost causes an acceptance problem for the current analysis. Figure 5.3 shows that a large percentage of the generated $\Lambda_{c}$ are booseted out of the front of the detector.

\section{$3.2 \Upsilon(4 S)$ and Continuum Events}

The data used for this analysis is taken from two center of mass energies, 10.58 $\mathrm{GeV}$, which is the mass of the $\Upsilon(4 S)$ resonance, and $10.54 \mathrm{GeV}$, which is sufficently far below the resonance to consist only of virtual photon decays to quarkantiquark pairs. This second dataset will be referred to as offpeak, and the quark 
jet events contained therin as continuum events. As the virtual photon crosssection changes little between 10.54 and $10.58 \mathrm{GeV}$, continuum events are also present in the onpeak dataset. The $\Upsilon(4 S)$ decays to a pair of $\mathrm{B}$ mesons, which are the subject of most of the physics analyses at BaBar, but we will be primarily interested in the continuum events. In order to increase our data sample in chapter 8 we will use both the onpeak and offpeak data samples, applying a 2.3 $\mathrm{GeV} / \mathrm{c}$ center of mass momentum cut to the $\Lambda_{c}$ in order to cut out any possible decays from B mesons.

\subsection{Silicon Vertex Tracker (SVT)}

The BaBar detector consists of five subdetectors arranged in concentric cylinders around the collision region. The innermost subdetector, the Silicon Vertex Tracker (SVT) is made up of five layers of double sided silicon detectors. The outer sides of each strip measure the $\phi$ coordinate of charged tracks, while the inner side measures $\mathrm{z}$. The resolution of individual hits is around $10 \mu \mathrm{m}$, which allows decay vertices to be measured with a resolution of $80 \mu \mathrm{m}$. This allows us to separate useful physics tracks from those created from interactions with the beampipe and detector material.

\subsection{Drift Chamber (DCH)}

The drift chamber consists of 40 layers of small hexagonal cells providing 40 spatial and ionization loss measurements on the charged tracks that traverse it. Each cell consists of a sense wire and 6 field wires with a potential difference of $1960 \mathrm{~V}$. Information on the z coordiante of the tracks is provided by stereo layers which are oriented a angles of $\sim 60$ mrad to the axial layers. The gas inside the 
chamber is a 80:20 mixture of helium:isobutane.

The primary purpose of the drift chamber is to provide precision measurements of the charged particles' momenta and angles. It is able to locate tracks with a precision of less than $.3 \mathrm{~mm}$ and measures the tracks' transverse momentum with a resolution of

$$
\frac{\sigma_{p_{t}}}{p_{t}}=.13+.45 p_{t} \%
$$

where $p_{t}$ is measured in $\mathrm{GeV} / \mathrm{c}$.

The drift chamber can also be used to calculate a particles velocity, and therefore mass, by measuring the amount of energy it loses to ionization as it traverses the gas. Particle identification using the DCH will be described in detail in the next chapter.

\subsection{Detector of Internally Reflected Cherenkov Radiation (DIRC)}

The DIRC utilizes fused silica bars, both as Cherenkov radiators and reflectors, which transfer the Cherenkov light cones emitted by charged particles from the detector to a large water filled expansion region called the standoff box. The standoff box is instrumented with 10,752 photomultiplier tubes. Each photomultipler hit can measure the Cherenkov angle with a resolution of about $10 \mathrm{mrad}$. A typical track will produce 20-65 hits allowing a Cherenkov angle measurement with a resolution of $2.5 \mathrm{mrad}$. Particle identification using the DIRC will be described in detail in the next section. 


\subsection{Electromagnetic Calorimeter (EMC)}

The Electromagnetic Calorimeter measures electromagnetic showers in thallium doped CsI crystals. This allows the detection of photons from $\pi^{0}$ and $\eta$ decays and from electromagentic and radiative processes. The energy resolution of the EMC is

$$
\frac{\sigma_{E}}{E}=\frac{.23}{E(G e V)^{\frac{1}{4}}} \oplus 1.85 \%
$$

It is not used in this analysis.

\subsection{Instrumented Flux Return (IFR)}

The steel flux return of the solenoid magnet is segmented into 18 plates varying in thickness from $2 \mathrm{~cm}$ in the innermost region to $10 \mathrm{~cm}$ at the outermost edge of the detector. The spaces between the plates are instrumented with resistive plate chambers (RPCs). When traversing the steel plates, strongly interacting hadrons will lose a greater amount of energy than muons. The difference in penetration depth allows the IFR to identify $1 \mathrm{GeV} / \mathrm{c}$ and greater muons with an efficiency of over $80 \%$ while misidentifying less than $10 \%$ of pions as muons. The IFR is also not used in this analysis. 


\section{CHAPTER 4}

\section{Particle Identification}

\subsection{Charged Particles}

The charged particle identification for this analysis uses information from two subdetectors; the drift chamber (DCH) and the detector of internally reflected Cherenkov radiation (DIRC). The drift chamber measures $\mathrm{dE} / \mathrm{dx}$ with a resolution of 7 percent which gives very good $\mathrm{K}-\pi$ separation below $.5 \mathrm{GeV} / \mathrm{c}$ and p-K separation below .7 GeV/c. The DIRC begins to see a sufficient number of photons for high efficiency identification of kaons and protons at around $1 \mathrm{GeV} / \mathrm{c}$ and $1.5 \mathrm{GeV} / \mathrm{c}$ respectively. At intermediate momenta, a linear combination of cuts from both subdetectors is necessary. This section will describe how these cuts were chosen.

\subsubsection{DCH and DIRC Likelihoods}

The drift chamber uses the total charge deposited in each drift cell to measure the energy loss of charged particles crossing its volume. The Bethe-Bloch formula;

$$
-\frac{d E}{d x}=\frac{4 \pi n z^{2} e^{4}}{m_{e} v^{2}}\left[\ln \frac{2 m_{e} v^{2}}{I\left[1-(v / c)^{2}\right]}-(v / c)^{2}\right]
$$

where $\mathrm{z}$ is the charge of in the incoming particle, $\mathrm{n}$ is the electron density in the gas, and I is its average ionisation energy; gives the theoretical energy loss, which is plotted for each particle type, along with the data in figure 4.1. This 


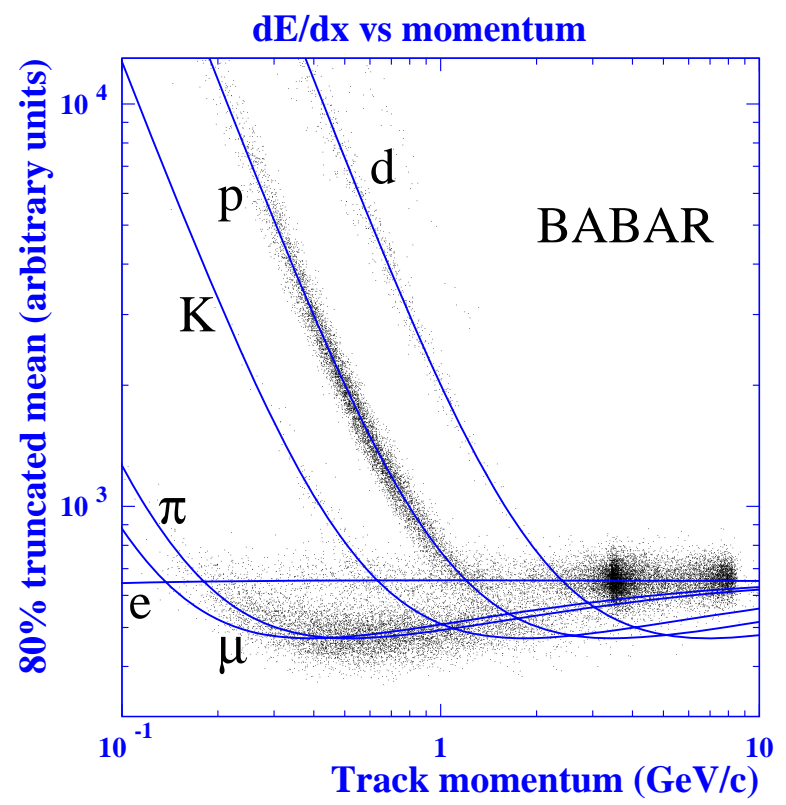

Figure 4.1: dE/dX vs. momentum in the drift chamber

plot shows that the $\mathrm{dE} / \mathrm{dx}$ values are well separated for different particle types at low momentum.

The DIRC identifies particles by their Cherenkov angle, which is determined by the velocity of the charged track in the fused silica bars.

$$
\cos \left(\theta_{c}\right)=\frac{1}{n \frac{v}{c}}
$$

where $\mathrm{n}$ is the index of refraction for fused silica (1.473).

The theoretical Cherenkov angles for $\pi, \mathrm{K}$, and p are ploted in figure 4.2 along with the output of the ring fitting algorithm for data. At low momentum, we are below the Cherenkov thresholds for kaons and protons, and so are unable to disinguish these particles. Comparing this to figure 4.1 one sees that the drift chamber should provide the best particle identification information up to about $.7 \mathrm{GeV} / \mathrm{c}$ and that the DIRC should be used for higher momenta. When the Cherenkov angles begin to converge around $3 \mathrm{GeV} / \mathrm{c}$ one could gain additional 


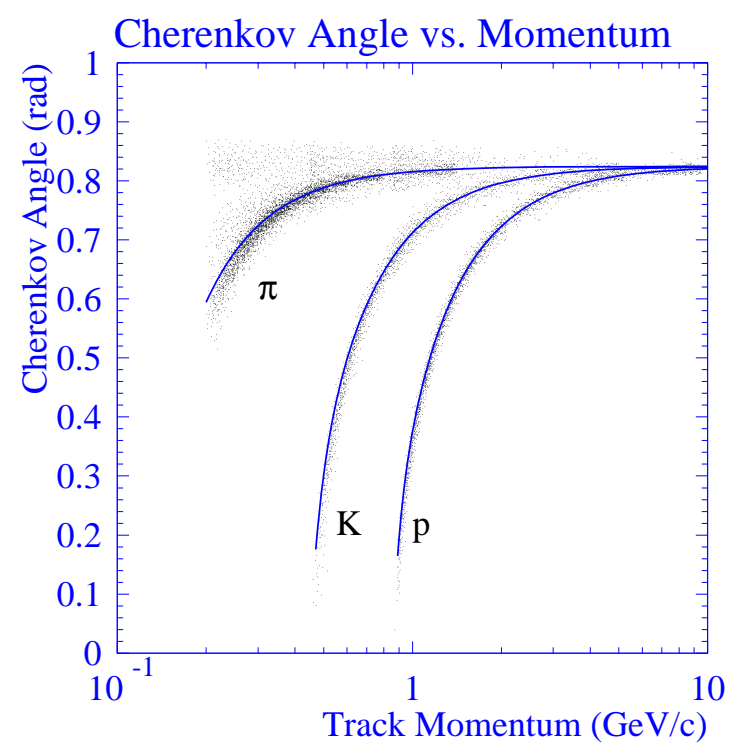

Figure 4.2: Cherenkov angle vs. momentum in the DIRC

information from the drift chamber. However, at the time these selectors were made, the Monte Carlo/data differences were better understood in the DIRC and so we chose to use only DIRC information at the highest momenta.

The cuts for particle identification are made on the output of the DIRC global likelihood algorithm, which looks at photomultiplier hits for the entire event and tries to determine if they are more likely to have come from a real track or from machine background, and, in the case of overlapping rings, which track they came from. The likelihood is defined to be the overall proability of there being $\mathrm{N}$ detected photoelectrons and those photoelectrons being distributed in the Cherenkov angle and time space as they were found. The input into the likelihood is the reconstructed Cherenkov angle of each PMT hit and its measured time. For each track, a likelihood for each of the five particle types is calculated while holding all other tracks at their current best hypothesis. The tracks are 
looped over a maximum of three times to insure that the backgrounds for each track have the best hypotheses for each background track. The global likelihood procedure gives better results than a track by track likelihood in the region just above threshold, where there are not always enough hits to get a good Cherenkov angle measurement. We are particularly interested in this region since it is where one sees the most structure in the efficiency vs. momentum plots.

\subsubsection{Optimization Method}

Figure 4.3 shows what the DIRC and drift chamber likelihoods look like for Monte Carlo pions and kaons in the momentum region $.4 \mathrm{GeV} / \mathrm{c} \leq \mathrm{p} \leq .9 \mathrm{GeV} / \mathrm{c}$. The $\mathrm{x}$-axis shows the difference in the logs of the DIRC global likelihood for kaons and pions. The y-axis is the log of the drift chamber likelihood for kaons minus that for pions. Thus true kaons are expected to show up in the top right corner of the plot, and pions in the bottom left corner. Particles pass the pion vs. kaon background cut if they are below the plotted line, which is defined by its slope and the offset of its intersection with the line $\mathrm{x}=\mathrm{y}$.

Plot a) shows particles with momenta $.4 \mathrm{GeV} / \mathrm{c} \leq \mathrm{p} \leq .45 \mathrm{GeV} / \mathrm{c}$. Most of the pions appear on the lower edge of the plot (note the log scale in $\mathrm{z}$ ) and almost all of the kaons have a postive drift chamber likelihood difference, so it it easy to separate the two using only drift chamber information. This corresponds to a horizontal line cutting the DCH/DIRC space. This momentum is below the kaon Cherenkov threshold, so the DIRC identifies everything as either a pion or undecided (the line at $\mathrm{x}=0$ ).

Plot b) shows $.55 \mathrm{GeV} / \mathrm{c} \leq \mathrm{p} \leq .60 \mathrm{GeV} / \mathrm{c}$. Most of the kaons have moved to the right edge, but because they are nearer to threshold than the pions and thus have fewer Cherenkov photons, their distribution has a longer tail. The pions at 


\section{DIRC and DCH Likelihoods for $\pi$ and $\mathrm{K}$}
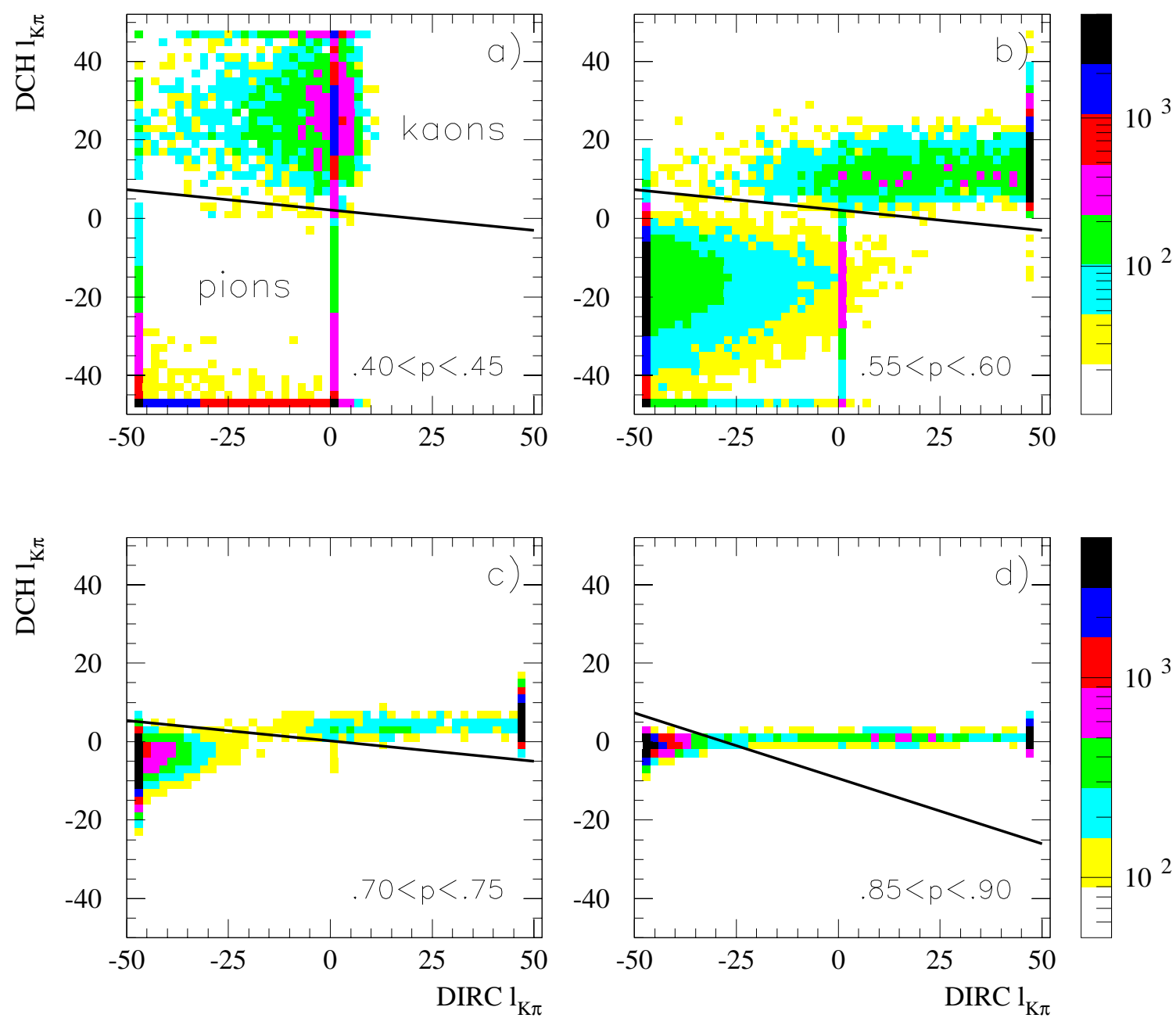

Figure 4.3: DCH vs. DIRC $\log$ (likelihood) differences for four different momentum regions. 
$\mathrm{x}=0$ are also not creating enough DIRC hits to get a good measurement. Here we need to use both detectors to optimize efficiency, but, because of the long tails in the DIRC distribution, the ideal slope for the cut is still very flat.

In c) and d) the particles can be well separated using only information from the DIRC. Because the distributions in DCH likelihood difference are so much narrower than those in the DIRC, the actual slope chosen for the cut does not matter much in this momentum range. However, since the DIRC distributions will narrow at higher momenta, the absolute value of the slope was chosen to increase linearly with momentum in this range.

Given a series of plots like those in figure 4.3, cuts were chosen to optimize the efficiency/misidentification for all momenta. As a first step, the slope of the cut which optimizes pion efficiency given that less than $1 \%$ of kaons are misidentified as pions was plotted in figure 4.4. Then a piecewise linear function was chosen to match the points, paying special attention to the region between .7 and 1.1 $\mathrm{GeV} / \mathrm{c}$. Above this, the efficiency is not a strong function of slope. Once the slope of the cut was chosen, the line was offset in varying amounts in order to see what the efficiency/misidentification tradeoff was in each momentum bin. The result is shown in figure 4.5, where pion efficiency increases in the positive y direction and kaon misidentification rate decreases with decreasing y. Again a piecewise linear function was chosen, giving a result of about $2 \%$ kaon misidentification over all momenta and $95 \%$ pion efficiency up to $1.5 \mathrm{GeV} / \mathrm{c}$.

Thus far, only pion efficiency with a kaon background has been considered. Similar plots were made for all three particle types versus the two backgrounds for each type. A particle passes the selection only if it passes both background cuts. In addition, very loose cuts were made to discriminate kaons and protons from electrons. 


\section{Maximizing $\pi$ Efficiency with $1 \% \mathrm{~K}$ Background}

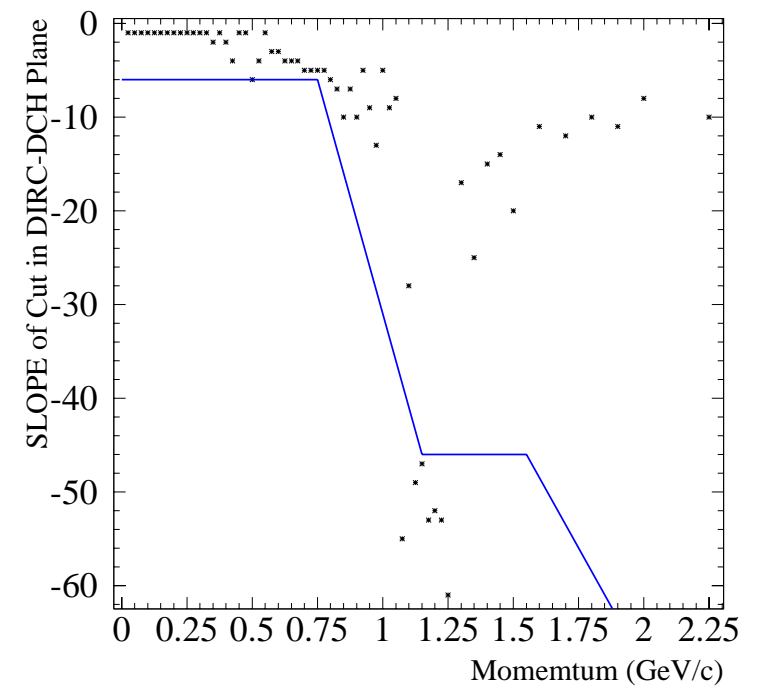

Figure 4.4: Slope of cut which maximizes pion efficiency given a $1 \%$ kaon background. Above $1 \mathrm{GeV} / \mathrm{c}$, efficiency is not a strong function of angle, and so the "best" points are scattered. 
Choosing Cut for $\pi$ Selection with K Background

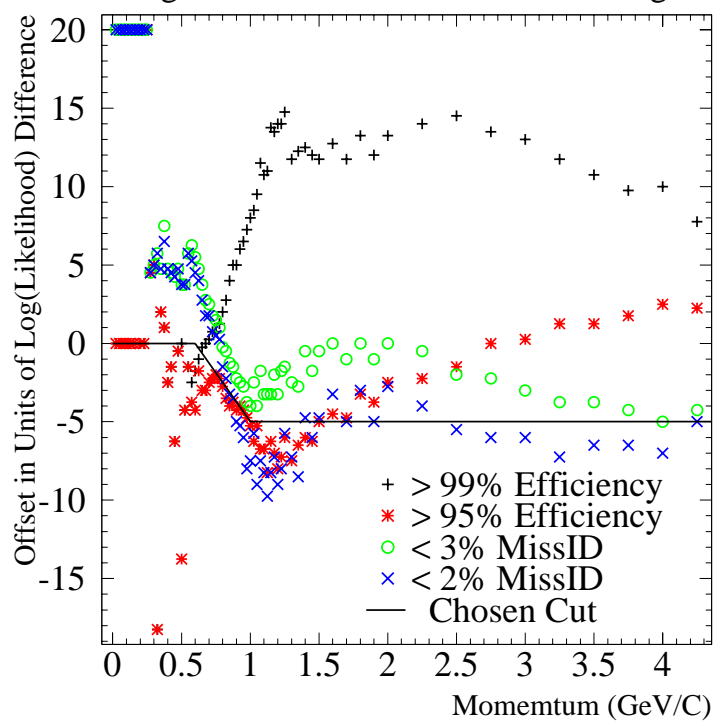

Figure 4.5: Offset of cut for different efficiency and missID rates

After optimizing the cuts with a Monte Carlo sample integrated over the entire theta range, it was found that the kaon and proton efficiencies dropped severely near $\cos \theta=0$, which corresponds to tracks hitting the DIRC bars normally (figure 4.6). When such tracks are just above the Chernkov threshold, very few of the photons are internally reflected in the DIRC bars. Thus the cuts needed to be reoptimized in this region (figure 4.7). The final selectors have different cuts for large and small $\cos \theta$ with a linear interpolation between them.

\subsubsection{Performance}

The MC efficiency matrix for the selectors is shown in figure 4.8 for four different theta regions in the lab frame. By design there is very little $\cos \theta$ dependence in the proton efficiency or in the pion and kaon efficiencies up to $2.5 \mathrm{GeV} / \mathrm{c}$. 


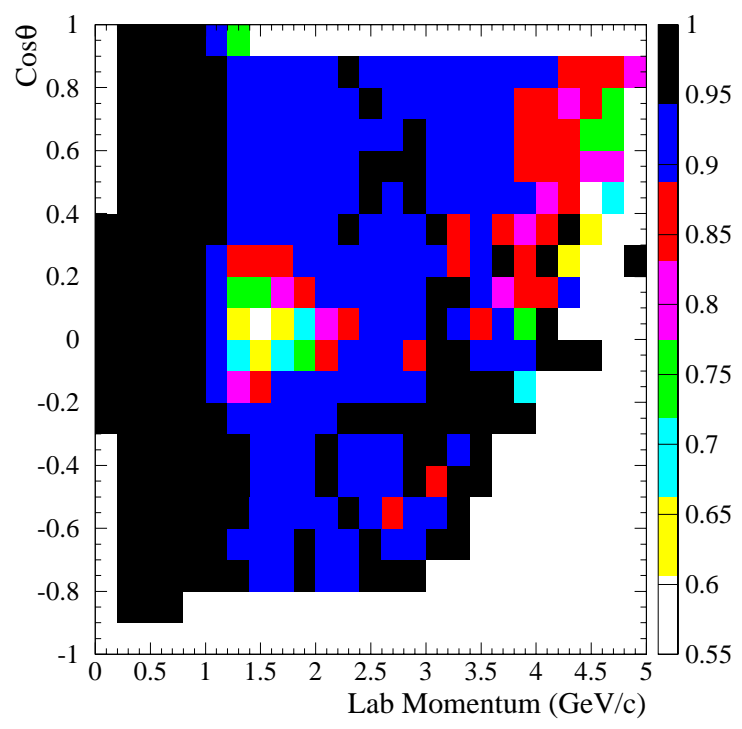

Figure 4.6: Hole in proton efficiency as a function of track angle and momentum due to non internally reflected Cherenkov photons

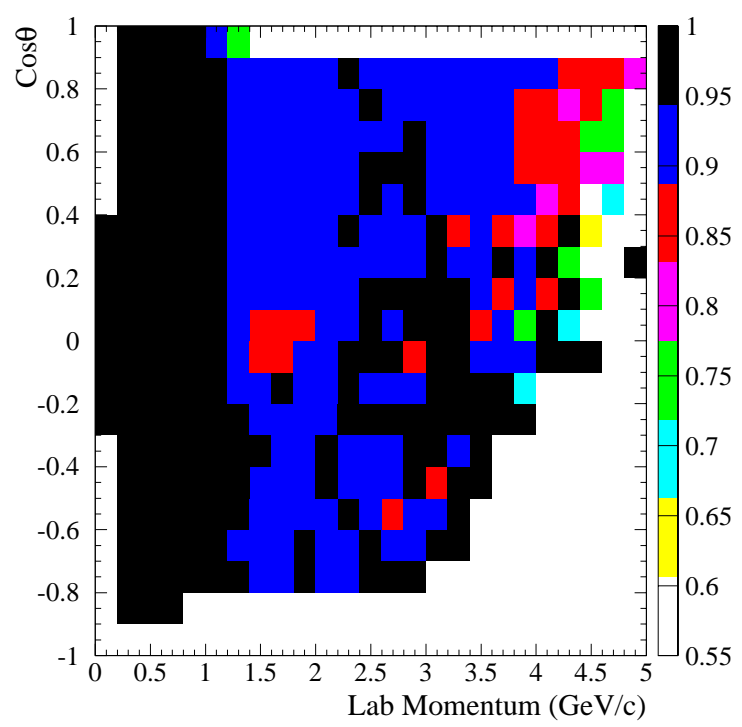

Figure 4.7: Proton efficiency after reoptimizing cuts 


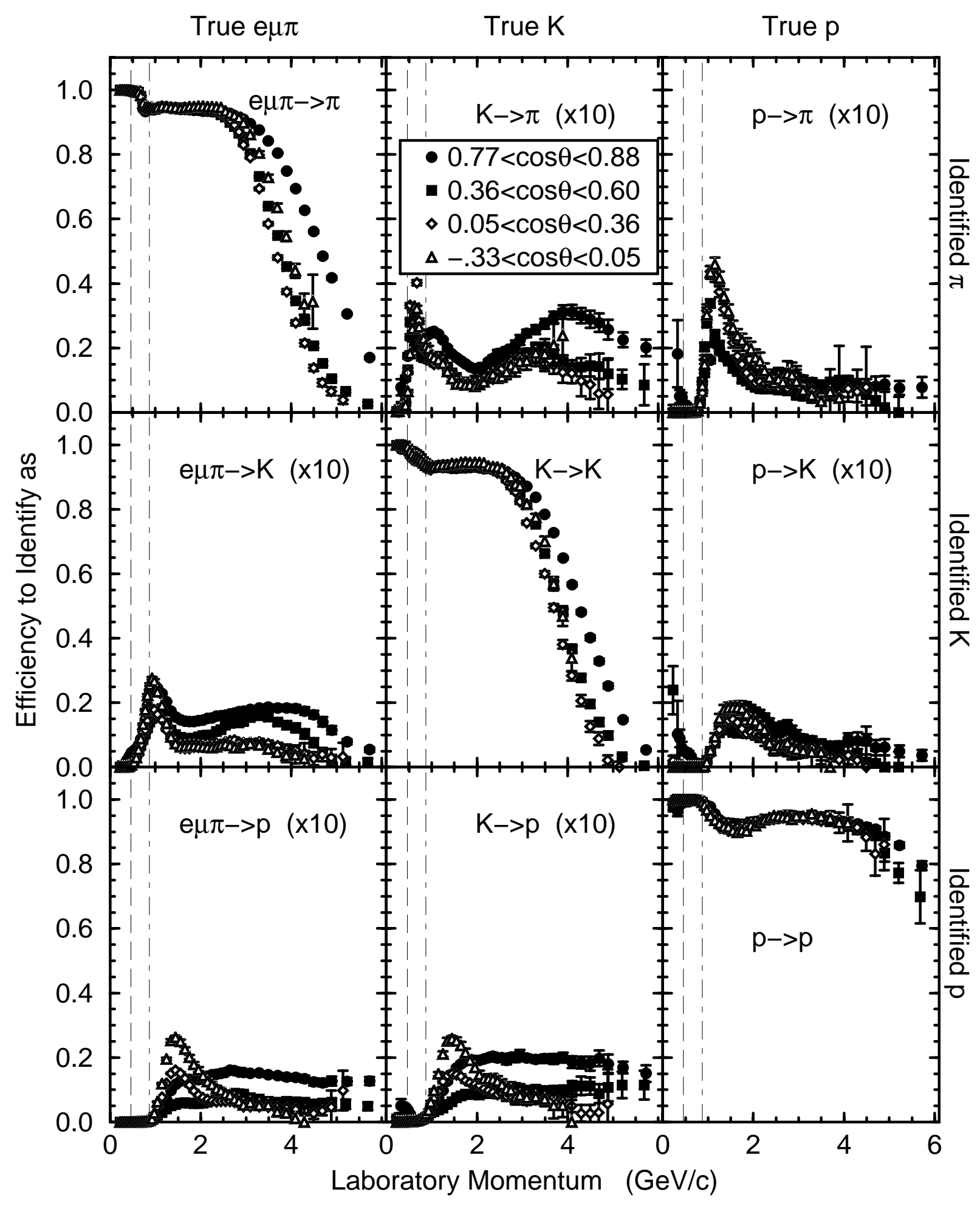

Figure 4.8: MC efficiency in 4 theta regions. 


\subsection{Neutral Particles}

In chapter 8 we will need to identify neutral $K^{0}$ mesons and $\Lambda$ baryons. Our goal is to get a good signal to noise ratio for the decays $\Lambda_{c} \rightarrow K^{0} p$ and $\Lambda_{c} \rightarrow \Lambda \pi$ so we will choose our cuts using the two- $\Lambda_{c}$ candidate dataset. In order to find $K^{0}$ mesons we first combine all sets of oppositely charged tracks with 10 or more drift chamber hits. If these tracks happen to be the daughters of a prompt $K^{0}$ then its flight length will be the distance between the point of closest approach of the two tracks and the global event vertex. We require that this quantity be less than $60 \mathrm{~cm}$. We also require that the $K^{0}$ candidate be moving away from the event vertex, and that the probability that the two charged tracks intersect, given by the standard BaBar vertexing algorithm, be greater than $1 \%$.

The invariant mass distribution of the $K^{0}$ candidates meeting these criteria is plotted as the black curve in figure 4.9. Candidates with a flight length greater than $2.5 \mathrm{~mm}$ are shown in red. In the plot on the right, the candidates which fail the $2.5 \mathrm{~mm}$ cut are also shown in red. Then we measure the cosine of the angle between the flight length vector and the $K^{0}$ candidate momentum and require that it be greater than .97. Candidates passing this cut are shown in green in the left plot of figure 4.9. Those which fail the cut are shown in the right plot.

The same procedure is carried out for the decay $\Lambda \rightarrow p \pi$, with the results shown in figure 4.10. Unlike in the case of the $K^{0}$, we use particle identification on the charged tracks. As a result, the flight length and angle cuts aren't really necessary and can take looser values of $.1 \mathrm{~mm}$ and .9 , respectively. 

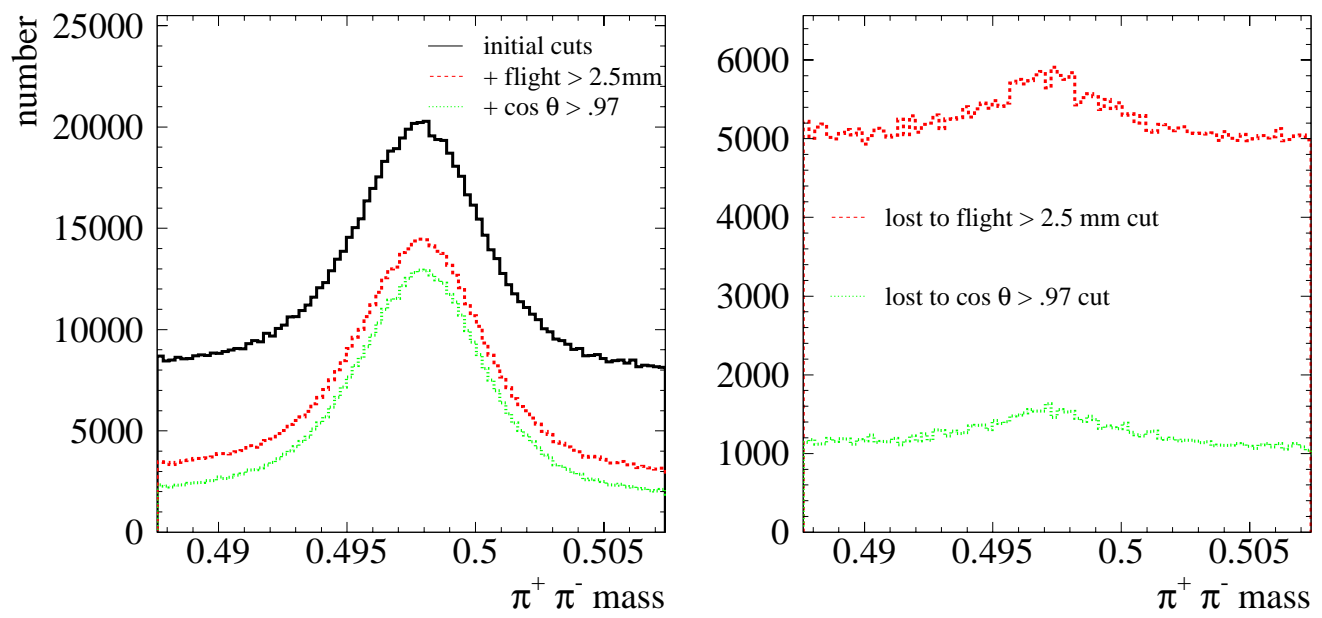

Figure 4.9: $K^{0}$ signal from the two- $\Lambda_{c}$ dataset. Effect of flight length cut and a cut on the angle between the flight length and momentum vectors. Particles thrown out with each cut are shown in the right plot.
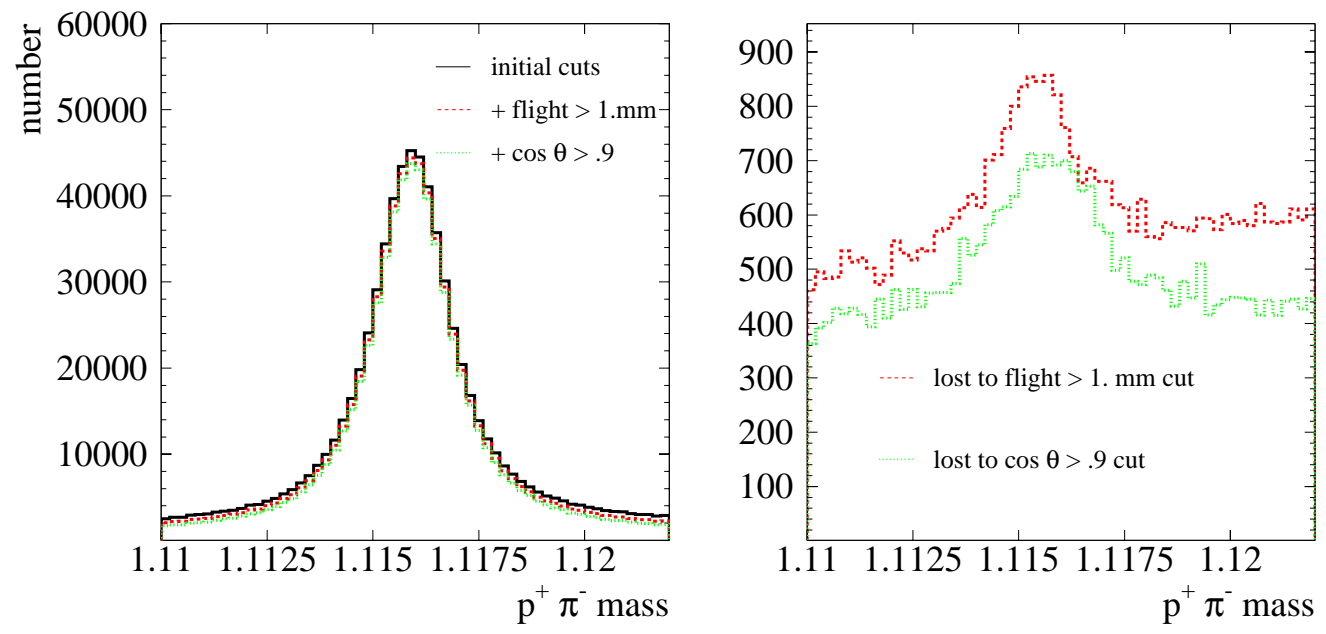

Figure 4.10: $\Lambda$ signal from the two- $\Lambda_{c}$ dataset. Effect of flight length cut and a cut on the angle between the flight length and momentum vectors. 


\section{CHAPTER 5}

\section{Reconstruction of $\Lambda_{c}$ 's}

This chapter will show how we reconstruct the decay $\Lambda_{c} \rightarrow \pi k p$, which we will be using for our measurement of the continuum $\Lambda_{c}$ spectrum at $10.54 \mathrm{GeV}$. We will estimate the efficiency of the reconstruction using Monte Carlo, and then apply $\mathrm{MC} /$ Data corrections for the particle identification and tracking.

\subsection{Event and Track Selection}

An inclusive hadronic spectrum measurement will usually use cuts on quantities like the second Fox-Wolfram moment, total detected energy in the event, or thrust in order to reduce backgrounds from gamma-gamma collisions, tau decays and Bhabhas. However, since none of these processes can easily create a charm quark, they should not be necessary. All of the standard multihadronic event selection cuts were made on the $\Lambda_{c}$ data, and none were seen to increase the signal to noise ratio.

A tight track selection has been applied in order to separate issues of particle identification from other detector questions. In particular, tracks were required to satisfy these criteria:

- Good angular resolution at the DIRC and a good measurement of $\mathrm{dE} / \mathrm{dx}$ in the DCH: 
- The track has more than 3 layers with SVT $z$ information and a total of at least 5 hits in the SVT.

- The track has at least 20 hits in the DCH.

- The track originates from the primary vertex:

- The distance of closest approach of the track to the beam in the transverse plane is less than $1 \mathrm{~mm}$. The $\Lambda_{c}$ lifetime of $.21 \mathrm{ps}$ means that a particle with maximum transverse momentum will only travel .15 $\mathrm{mm}$.

Lambda $\mathrm{C}$ candidates are formed by combining the four-vectors of three tracks passing the tracking cuts and identified as $\mathrm{p}, \mathrm{k}$ and $\pi$ by the algorithm described in chapter 4 . Once a candidate is selected, the momentum of the resulting $\Lambda_{c}$ is calculated by adding the momenta of the three daughter tracks at their point of closest approach to the event vertex. Because the Kalman track fitter used by the standard BaBar code assumes a pion mass for all tracks, a correction algorithm written by Bill Dunwoodie is used to correct the kaon and proton momenta for the energy losses they experience when traversing the detector material.

Algorithms have been written to calculate the probability that the three daughter tracks are coming from a common vertex. However, because of the assumed pion mass in the fitter, they do not give reliable results at low momentum. Since the continuum $\Lambda_{c}$ 's decay so closely to the event vertex, the distance of closest approach cut described above should be sufficient for removing backgrounds associated with material interactions.

In order for one of the daughter tracks to have the required 20 drift chamber hits it must have more than about $0.12 \mathrm{GeV} / \mathrm{c}$ of transverse momentum and fall in the angular range $-.85 \leq \cos \theta_{\text {lab }} \leq .95$. We define this cut as the acceptance 
and plot its efficiency vs. the $\Lambda_{c}$ lab momentum in the first row of figure 5.1 and vs. the $\Lambda_{c}$ angle in the lab frame in the first row of figure 5.2. The fastest $\Lambda_{c}$ tend to be boosted towards the front of the detector, which increases the probability that one of the daughters will not have enough DCH hits. Slower $\Lambda_{c}$ are less likely to pass the transverse momentum cut. Since the pion takes up a small fraction of the parent momentum, it is less likely than the heavier particles to follow the

$\Lambda_{c}$ direction and therefore its acceptance efficiency is less strongly correlated to the $\Lambda_{c}$ momentum vector.

The second row of figures 5.1 and 5.2 shows the tracking efficiency, which is a combination of the efficiency to match a reconstructed daughter track to the true tracks used to calculate the acceptance, and the tracking cuts described above. Because the tracking requirements are less likely to be met near the edge of the acceptance, the shape of the tracking efficiency curves is similar to that of the acceptance efficiency curves.

The third row of figures 5.1 and 5.2 shows the particle identification efficiency for tracks that passed the tracking cuts. Fast tracks are more likely to be misidentified because of overlapping Cherenkov angle distributions. Backwards moving tracks are slower because of the boost and easily identifiable with the drift chamber.

\subsection{Lab Frame Efficiency Map}

The goal of the first part of the analysis is to measure the $\Lambda_{c}$ momentum spectrum in the center of mass frame, but because the detector efficiencies depend on variables in the lab frame, the efficiency corrections will be done in the lab frame and then the corrected number of $\Lambda_{c}$ will be boosted into the center of mass 

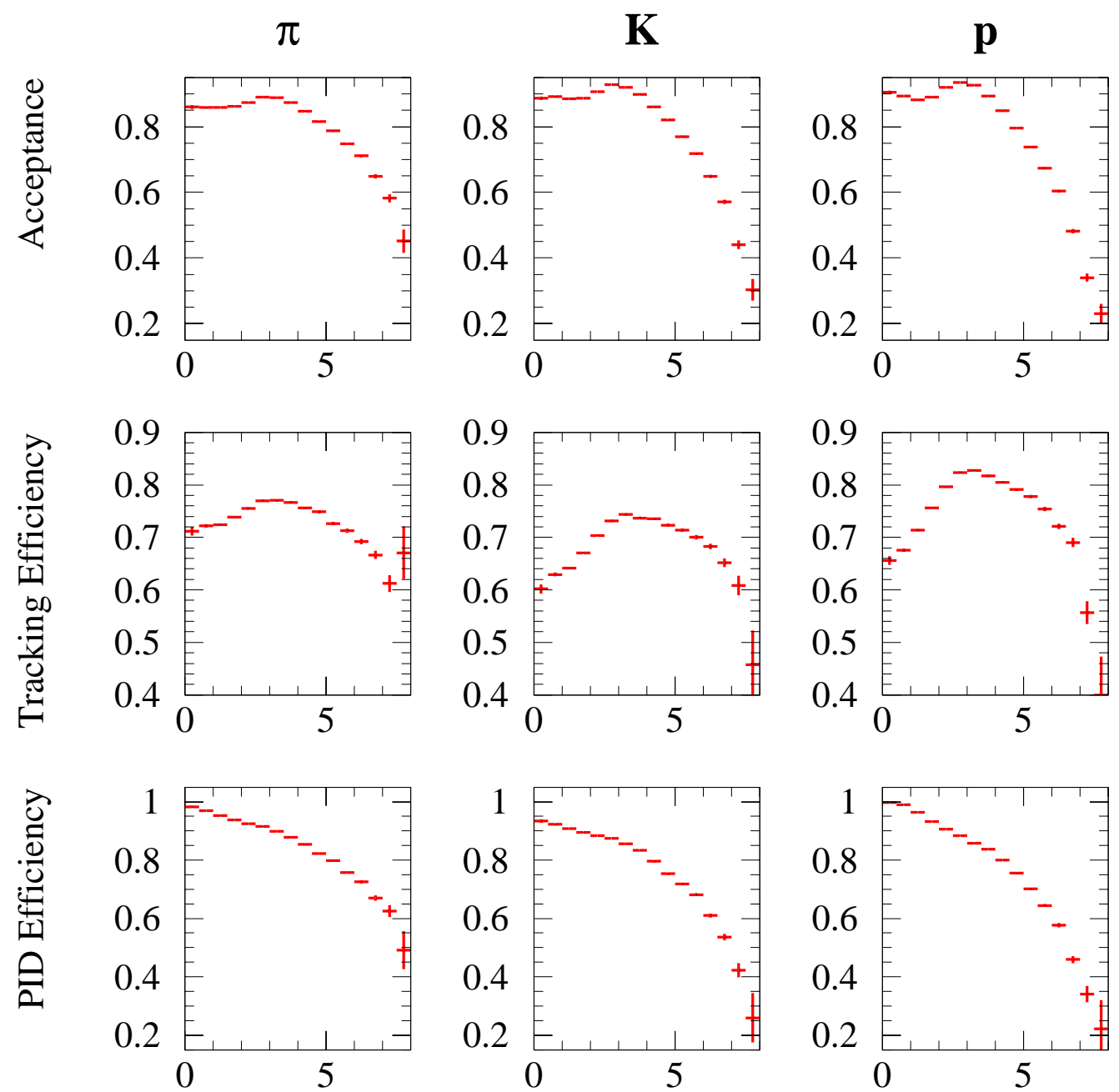

$\Lambda_{\text {c plab }}$

$\Lambda_{\mathrm{c}} \mathrm{p}_{\text {lab }}$

$\Lambda_{\mathrm{c}} \mathrm{p}_{\mathrm{lab}}$

Figure 5.1: Acceptance, Tracking Efficiency, and PID efficiency for the daughter tracks of a Monte Carlo $\Lambda_{c}$ with a particular lab momentum. 

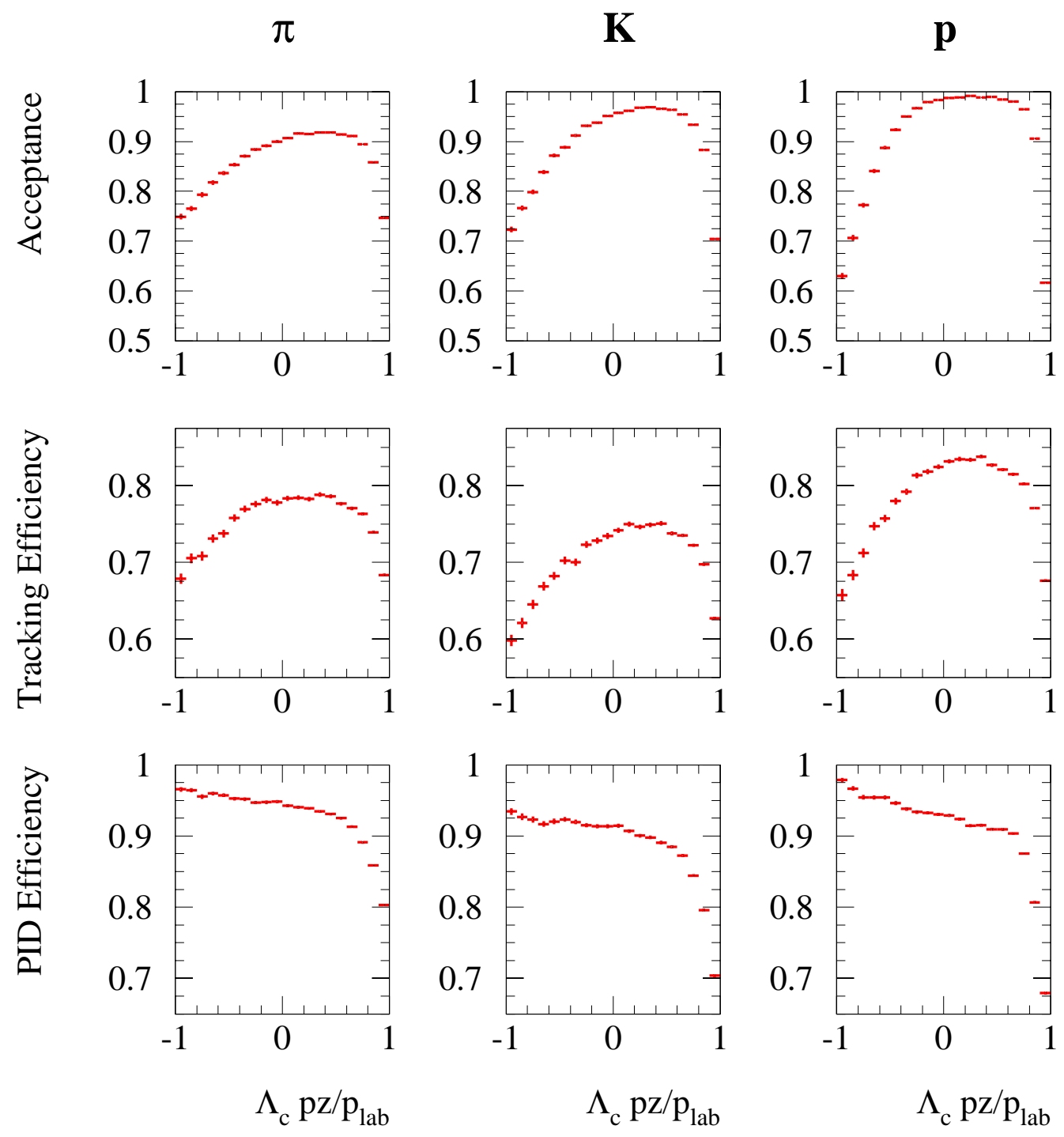

$\Lambda_{\mathrm{c}} \mathrm{pz} / \mathrm{p}_{\mathrm{lab}}$

Figure 5.2: Acceptance, Tracking Efficiency, and PID efficiency for the daughter tracks of a Monte Carlo $\Lambda_{c}$ with a particular lab angle. 
frame. There they will be put into bins of momentum and the invariant mass distribution will be fit to a sum of gaussians with polynomial background.

The Monte Carlo efficiency correction has two steps. First, Monte Carlo is used to make an efficiency map as a function of $\Lambda_{c}$ momentum and angle in the lab frame. This efficiency includes track finding, PID and reconstruction of the $\Lambda_{c}$ with a mass within $50 \mathrm{MeV}$ of the PDG value.

The second step is to extrapolate the $\Lambda_{c}$ yield to the region outside of $-0.8 \leq$

$\cos \theta_{C M} \leq 0.3$, where the efficiency is too low to get a reliable measurement. This must be done in bins of momentum, as slower $\Lambda_{c}$ are more likely to have come from events with a radiated hard gluon, which will smooth the angular distribution from the $1+\cos ^{2} \theta$ seen in more 2-jet like events. This can be seen in figure 5.3, which shows the Monte Carlo $\cos \theta_{c m}$ distribution for slow and fast $\Lambda_{c}$. Because the Monte Carlo calculates the initial stage of the parton shower perturbatively, it is expected to correctly model the distribution of hard gluons. The effect of hadronization on $\cos \theta_{c m}$ is small, so no data/MC correction is needed for this extrapolation. Later we will show that the extrapolation is consistant with data by doing the analysis separately in 6 bins of $\cos \theta_{c m}$ (figure 6.13).

\subsubsection{Making the Efficiency Map}

Because we are limited to $473 \mathrm{~K}$ continuum and $64 \mathrm{~K} \mathrm{~B}$ decay $\Lambda_{c}$ Monte Carlo events we must be sure to make the efficiency map binning large enough to get sufficient statistics so as not to dominate the other errors in the analysis. At the same time, care must be taken to reduce the bin size in regions of large curvature, so as to match the true efficiency as closely as possible. The following procedure was written in order to optimize the tradeoff between these two sources of error. 


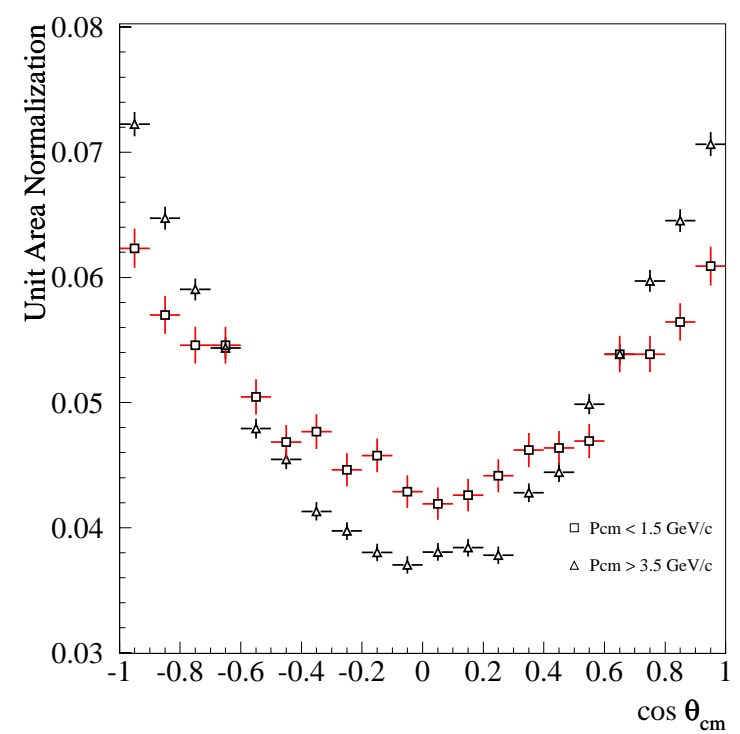

Figure 5.3: CM angular distribution for fast and slow $\Lambda_{c}$

First, the Monte Carlo was partitioned into a 300x300 matrix with $-1 \leq$ $\cos \theta_{l a b} \leq 1$ and $0 . \leq P_{l a b} \leq 8$.. The distribution of generated $\Lambda_{c}$ 's is shown in figure 5.4a. The large effect of the boost is visible in the $\cos \theta$ asymmetry. $\Lambda_{c}$ from $\mathrm{B}$ decays are visible as a separate distribution peaking around $2 \mathrm{GeV} / \mathrm{c}$. The black line in the figure 5.4 plots shows the location of the $-0.8 \leq \cos \theta_{C M} \leq 0.3$ cut and the kinematic upper limit for momentum. The rightmost section of the line shows $\Lambda_{c}$ with $4.75 \mathrm{GeV} / \mathrm{c}$ momentum in the center of mass. At the lowest point, we see that a center of mass momentum of $4.75 \mathrm{GeV} / \mathrm{c}$ and $\cos \theta_{C M}$ of -0.8 correspond to $3.2 \mathrm{GeV} / \mathrm{c}$ and -0.45 in the lab frame. Following the black line up and to the left, we see that as the center of mass momentum drops, the particles are boosted more and more towards the front of the detector until we reach the point where stationary center of mass particles are boosted to a lab $\cos \theta$ of one. The top section of the black line shows a similar behavior for the $\cos \theta_{C M}=0.3$ 

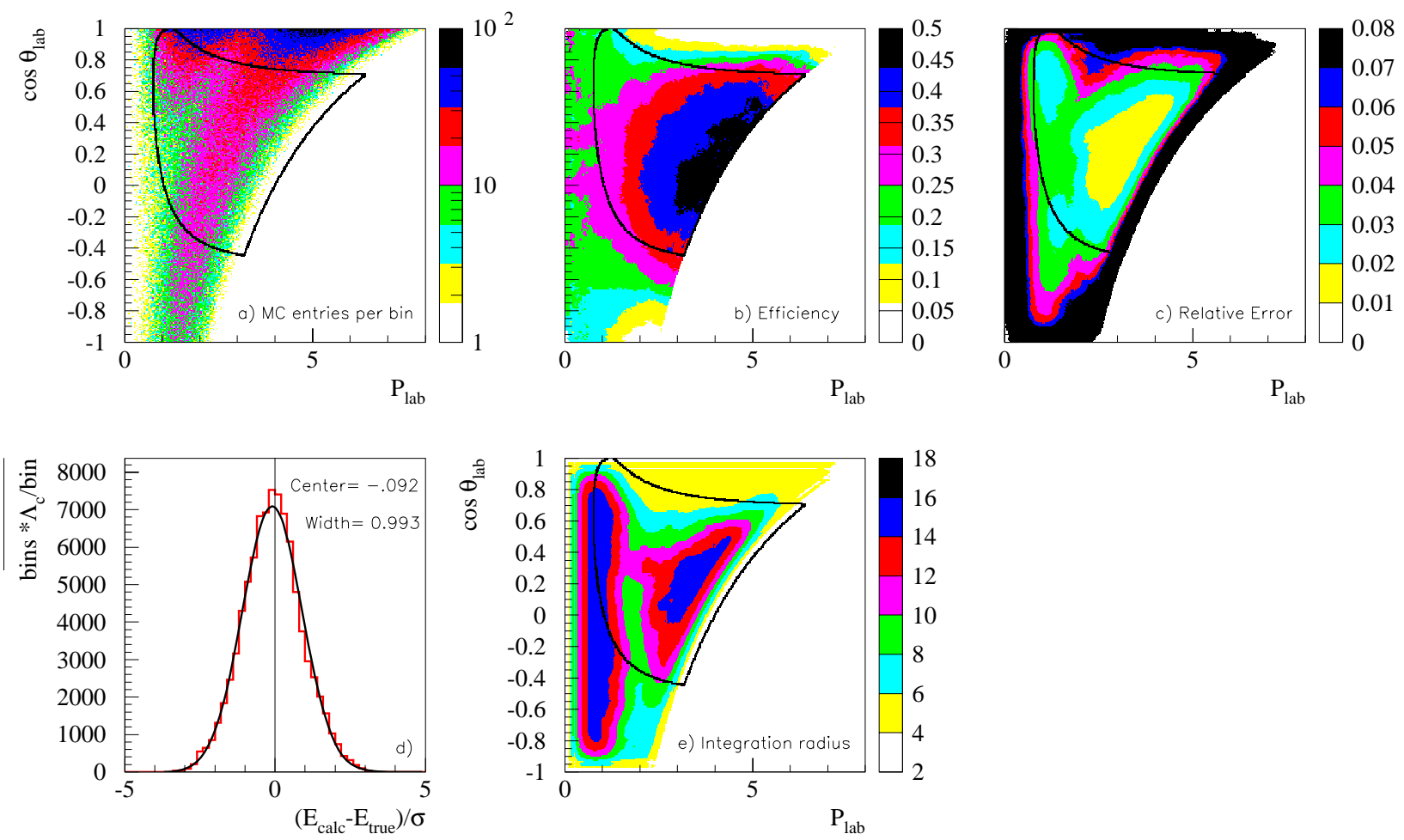

Figure 5.4: a) distribution of generated $\Lambda_{c}$ in the lab frame. The black line shows our acceptance cut. b) Reconstruction efficiency. c) Relative error on the efficiency. d) Efficiency pull distrubution. e) Radius of the circle over which entries are integrated in order to calculate the efficiency. 
particles. Figure $5.4 \mathrm{~b}$ shows that the large number of particles with $\cos \theta_{C M} \geq .3$ are reconstructed with a very low efficiency which varies rapidly with $\cos \theta_{l a b}$ so we are better off not trying to include them in the analysis.

The first step in calculating the efficiency is to assign a radius of integration for each of the 90,000 bins. This defines a circular region in the efficiency map, over which the Monte Carlo entries are integrated, in order to reduce the statistical error. This radius is initially set to 15 bins and allowed to vary by $10 \%$ with each iteration of the procedure. Bins near the kinematic limits for $\Lambda_{c}$ production have their radius of integration reduced until it no longer crosses into the unallowed region. The efficiency and statistical error for each bin is calculated using all of the generated $\Lambda_{c}$ that fall within the radius of integration of that particular bin. Secondly, extrapolations to all bins with a statistical error of greater than 7 percent are performed. Looking at figure $5.4 \mathrm{c}$, we see that this is only necessary for the fastest $P_{c m}$ bins, and a small number of bins between the continuum and $\mathrm{B}$ decay distributions at high $\cos \left(\theta_{l a b}\right)$. The direction of extrapolation is determined by looking at the status of neighboring bins. It is chosen to be in the direction opposite the neighbor bins which are closest to the kinematic limits of the $\Lambda_{c}$ distribution. A number of linear extrapolations are performed using sets of points at a distance of 16, 12, 8 and 4 bins from the current bin, and within 45 degrees of the calculated extrapolation direction. The results of these extrapolations and their errors are then averaged.

The efficiency in the extrapolated region is the error weighted average of the extrapolation and the original value. In order to ensure a smooth transition, the weights on the extrapolated values are linearly phased in as the statistical error on the original efficiency goes from 7 to $10 \%$. The final values of the efficiency and relative errors can be seen in Figure 5.4 b\&c. 
After each iteration of the efficiency calculation, the efficiencies of the bins centered on the edge of the circle defining the integration region of each bin are compared with that of the central bin. If the average of the absolute value of the difference is greater than twice the expected statistical fluctuation, then this is a sign that the efficiency map might have curvature in this region, and the radius of integration of the bin is reduced by $10 \%$.

After about 20 iterations, the radii of integration reach their optimal values and a final result is attained. The final values of the radii of integration are shown in Figure 5.4e. The radii become very small in the high $\cos \theta_{l a b}$ region, because the algorithm is trying to match the curvature of the efficiency by using smaller bins. The radii are also small in the region around $2 \mathrm{GeV} / \mathrm{c}$ because we have high statistics here, and don't need to integrate over a large region to get a good measurement.

As a consistancy check on the size of the reported uncertainties, 500K Monte Carlo events are generated using the just calculated efficiency map and an input momentum spectrum similar to that of the data. The efficiency is then recalculated and compared with the 'true' values used for the input. The pull of each bin is plotted, weighted by the number of particles in the bin. The Gaussian sigma of the resulting plot is 1.2 , so the errors on the original efficiency map are multiplied by this amount in order to give a self-consistant result. The pull distribution after rescaling the errors is shown in Figure 5.4d along with the best fitting Gaussian. This plot shows that the calculated efficiency is shifted down by about .09 standard deviations. The calculated efficiencies are expected to be slightly lower than the true values because we are integrating over a true efficiency with negative curvature. However, since the effect of this bias on the total yield is only $0.22 \%$, it is negligable compared to the other normalization errors. 


\subsubsection{Data/MC corrections}

Data/Monte Carlo differences in particle identification and tracking have been previously studied at BaBar. This section will show the corrections needed to be made to our Monte Carlo efficiency.

The efficiency and misidentification rates of the particle identification algorithm described in chapter 4 were studied in detail for the inclusive $\pi \mathrm{K} \mathrm{p}$ spectrum measurements [?]. The following decay channels were used to study data/MC differences:

- $K_{s}^{0} \rightarrow \pi^{+} \pi^{-}$: this is a very clean sample of pions with high statistics at low momenta;

- $D^{\star} \rightarrow D^{0} \pi_{S} \rightarrow K \pi \pi_{S}$ : this sample provides a source of both pions and kaons over a wide momentum range;

- $\Lambda \rightarrow \pi p$ : this yields a clean sample of protons over the entire momentum range of the analysis, as well as another sample of soft pions;

- $\tau \rightarrow 1$ or 3 charged tracks: this yields a sample that is predominantly pions and leptons and covers the very high momentum range;

- $\phi \rightarrow K^{+} K^{-}$: this gives a complementary source of kaons over a wide momentum range.

The efficiency corrections for the three daughter tracks of the $\Lambda_{c}$ are multiplied to get the total particle identification correction. This is shown as a function of $\Lambda_{c}$ center of mass momentum in figure 5.5. The correction is typically less than $5 \%$, but can be larger when one of the daughter tracks has a high momentum in the lab frame. The uncertainty of the correction, shown in the top left plot, is around $2 \%$. 

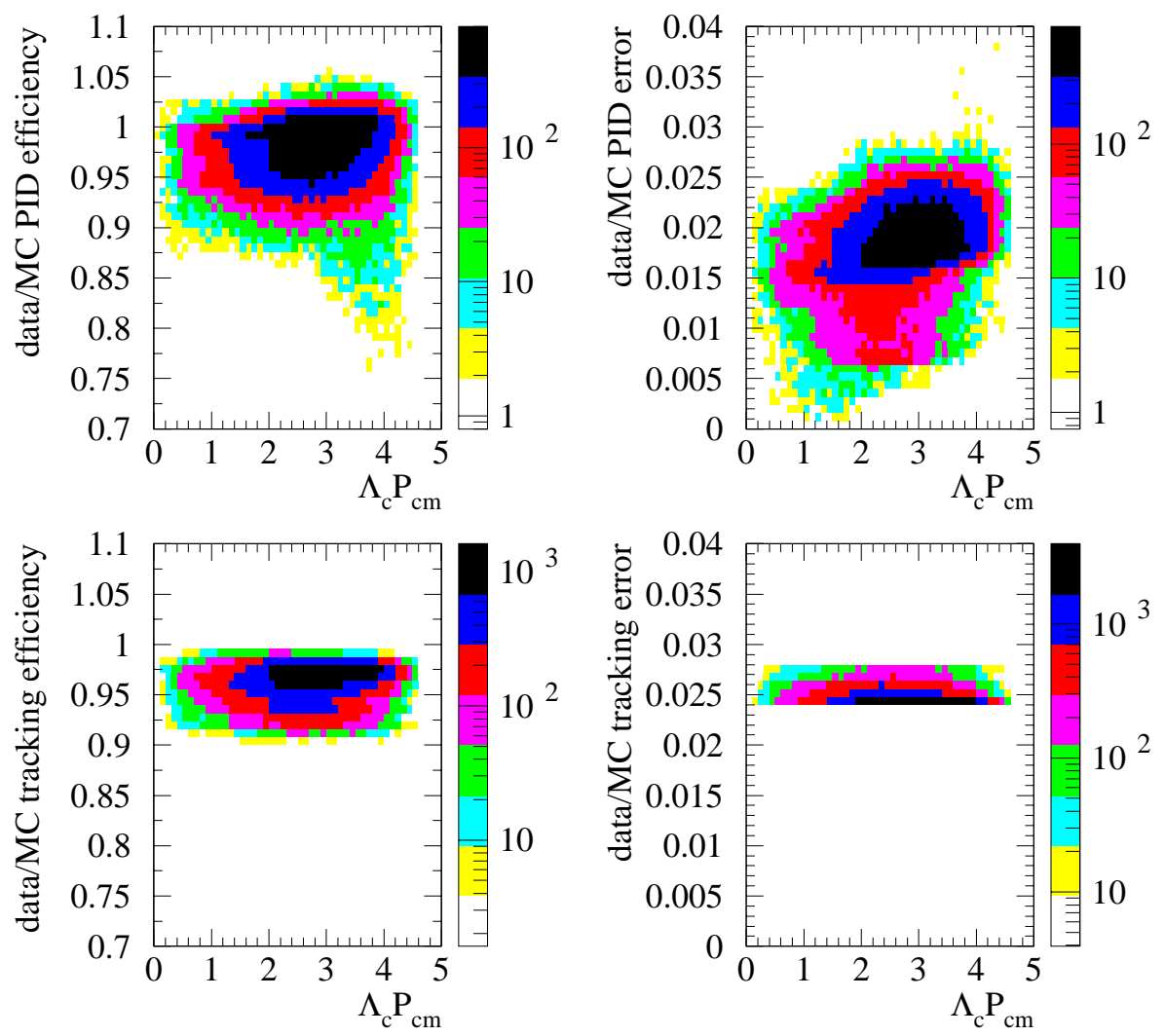

Figure 5.5: Data/MC Corrections and errors on the corrections for PID and tracking

The result of the standard BaBar tracking corrections and an additional correction due to missing $\Delta$ resonances in the detector material simulation [22] is shown in the bottom two plots. The error on the correction for each track is about $0.8 \%$ correlated over all momenta. For very soft pions it goes up to $1.3 \%$. The total tracking error is the sum of the errors for the three daughter tracks. 


\section{CHAPTER 6}

\section{Measurement of the $\Lambda_{c}$ Momentum Spectrum}

In this chapter we describe the measurement of the $\Lambda_{c}$ momentum spectrum. We describe how the invariant mass histograms are fit to extract the $\Lambda_{c}$ signal, calculate various systematic errors, and check to see that the results are consistant over different angular regions and data taking times. Finally, we present our results.

\subsection{Signal Extraction}

After applying the efficiency and data/MC corrections described in the previous chapter, the $\Lambda_{c}$ candidates are boosted into the center of mass frame, where they are divided into 19 momentum bins of $250 \mathrm{MeV} / \mathrm{c}$ width. The final bin ends at $4.75 \mathrm{GeV} / \mathrm{c}$ which is the kinematic limit. Because an event containing a $\Lambda_{c}$ must contain another c quark and baryon, the fastest possible $\Lambda_{c}$ would come from the decay virtual photon $\rightarrow \Lambda_{c}^{+}+\bar{\Lambda}_{c}^{-}$. Although, as we will see in chapter 8 this exclusive decay mode is never seen, it sets the scale for $p_{\max }=\sqrt{s / 4-m^{2}}$. In each momentum slice, the $\pi+\mathrm{K}+\mathrm{p}$ invariant mass is divided into 420 bins, $2.23 \leq$ mass $\leq 2.335 \mathrm{GeV} / \mathrm{c}$. Chosing a number of bins with a large number of different prime factors $(420=2 * 2 * 3 * 5 * 7)$ allows us to combine bins in several different ways (24) without rerunning the analysis or changing the size of the sidebands. The invariant mass bins in each momentum slice are combined until there are at least 
10 raw entires in each mass bin. This number is sufficiently high to allow us to use symmetric upper and lower error bars for a Poissonian distribution. Each $\Lambda_{c}$ is weighted by the correction determined in the previous section, and the statistical error for each invariant mass bin is taken to be the square root of the sum of the squares of the weights.

In order to calculate the systematic errors, the tracking, particle ID and Monte Carlo errors are added in quadrature and then added to the signal in each of the invariant mass bins. The resulting histogram is refit and the difference in the signal+error and signal only fits are taken as the systematic error.

\subsubsection{Full Dataset Study}

Our primary interest in the first part of this analysis is to study $\Lambda_{c}$ production in jets using the $9.460 \mathrm{fb}^{-1}$ of offpeak data taken at a center of mass energy of 10.54 $\mathrm{GeV}$. We also have $80.753 \mathrm{fb}^{-1}$ of onpeak data taken at $10.58 \mathrm{GeV}$. This dataset was taken just above $b \bar{b}$ threshold and so is contaminated by non-jet $B \bar{B}$ events , which will be studied in a future analysis, but it will also serve two purposes for the present study.

First, because $\Lambda_{c}$ from B decays cannot exceed a center of mass momentum of $2.3 \mathrm{GeV} / \mathrm{c}$, we can use the shape of the onpeak momentum spectrum above this value as a cross check for our offpeak measurement. Secondly, because the onpeak sample has more statistics, we can use it to study the position and resolution of the $\Lambda_{c}$ mass peak. By fixing the location and width of the mass peak in the offpeak fits to the values obtained using the entire dataset, we should be able to get a better measurement. 
The parameters of the fitting function are chosen by $\chi^{2}$ minimisation

$$
\chi^{2}(a, b, c, \ldots)=\sum_{\text {mass bins }} \frac{\left(\int_{b i n} f(a, b, c, \ldots, m) d m-\sum_{i} w_{i}\right)^{2}}{\sum_{i} w_{i}^{2}}
$$

where $\mathrm{a}, \mathrm{b}, \mathrm{c}, \ldots$ are the parameters of the fitting function, $w_{i}$ is the weight of each data entry, which is equal to the reciprocal of the reconstruction efficiency, and the sum is done over mass bins within $15 \mathrm{MeV}$ of the PDG $\Lambda_{c}$ mass.

For a given detector region, we expect the resolution function to be a Gaussian, but, because our center of mass momentum bins are actually integrating over large detector regions, a sum of two or more Gaussians may be necessary. We begin by fitting the entire dataset with a single Gaussian and 2nd order polynomial background. The squares in figure $6.1 \mathrm{a}$ show the $\chi^{2}$ per degree of freedom. When the same data is refit using a sum of two Gaussian for the signal (the triangles in figure 6.1a), the results are improved in the region $1.75 \leq P_{C M} \leq 4$. Because we have less statistics outside of this range, we are better off using just a single Gaussian. The fits using the sum of two Gaussians in this region and a single Gaussian everywhere else are shown in figure 6.2

Figure $6.1 \mathrm{~b}$ ) shows the value of the center of the $\Lambda_{c}$ mass distribution as a function of center of mass momentum. Before correcting for the particle energy loss due to interactions with the detector material, there was about a $5 \mathrm{MeV}$ shift at low momenta. After the correction there is still a slight slope. There is a discontinuity in the Monte Carlo at $1.5 \mathrm{GeV} / \mathrm{c}$ caused by the difference between the continuum and $\mathrm{B}$ decay $\Lambda_{c}$ distributions. Because the $\mathrm{B}$ decays are isotropic in the center of mass, their boosted momentum distribution in the lab frame looks different from a jet-like angular distribution of the same center of mass momentum.

Figure 6.1c) shows the width of the $\Lambda_{c}$ mass fits. The mass distribution is wider at high momentum because of decreased momentum resolution. Again 

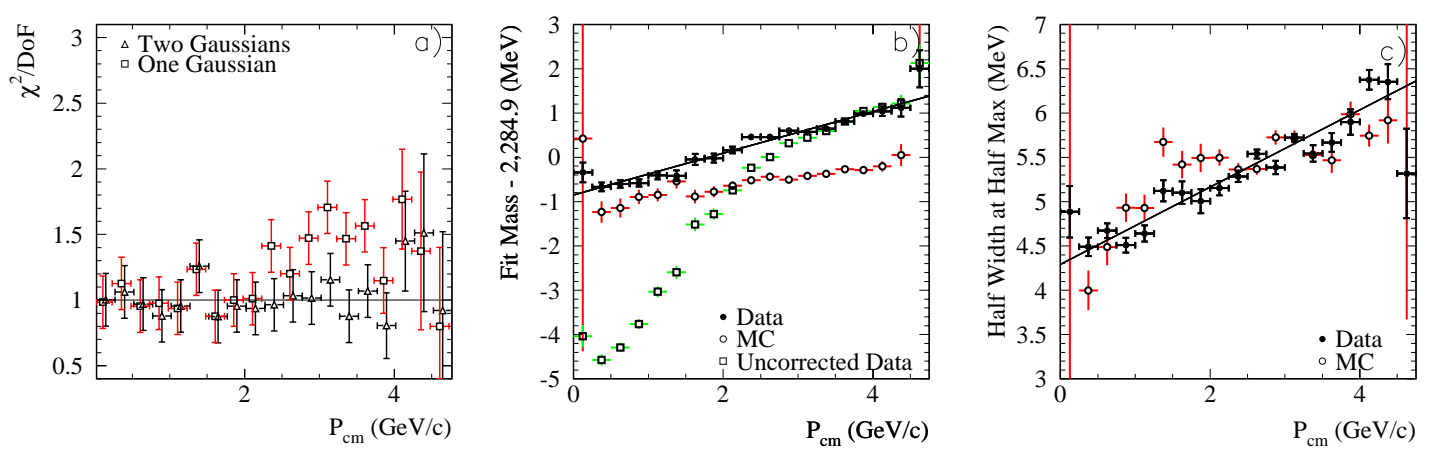

Figure 6.1: Fits using the entire dataset. a) $\chi^{2}$ for one and two Gauss fits. b) Mass shift vs. momentum. c) Width of the invariant mass distribution vs. momentum.

there seems to be a difference between the behavior of $\mathrm{MC} \Lambda_{c}$ from $\mathrm{B}$ and those from the continuum.

\subsubsection{Fitting the Offpeak Data}

The continuum $\Lambda_{c}$ measurement is done using only the offpeak data. Because we now have less statistics, the following changes are made to the fits.

- The background in momentum slices one, eighteen and nineteen is changed from quadratic to linear.

- The smallest number of raw entries in an invariant mass bin for momentum slices 17,18 , and 19 is 8,3 , and 4 respectively. In these slices, the absolute errors in bins with less than 7 raw entries are scaled up to what they would be if there were 7 entries with the same efficiency as the existing entries.

The mass and widths of the Gaussians are fixed to the values obtained in the full dataset study. The results of the fits are shown in figure 6.3. Because of low 

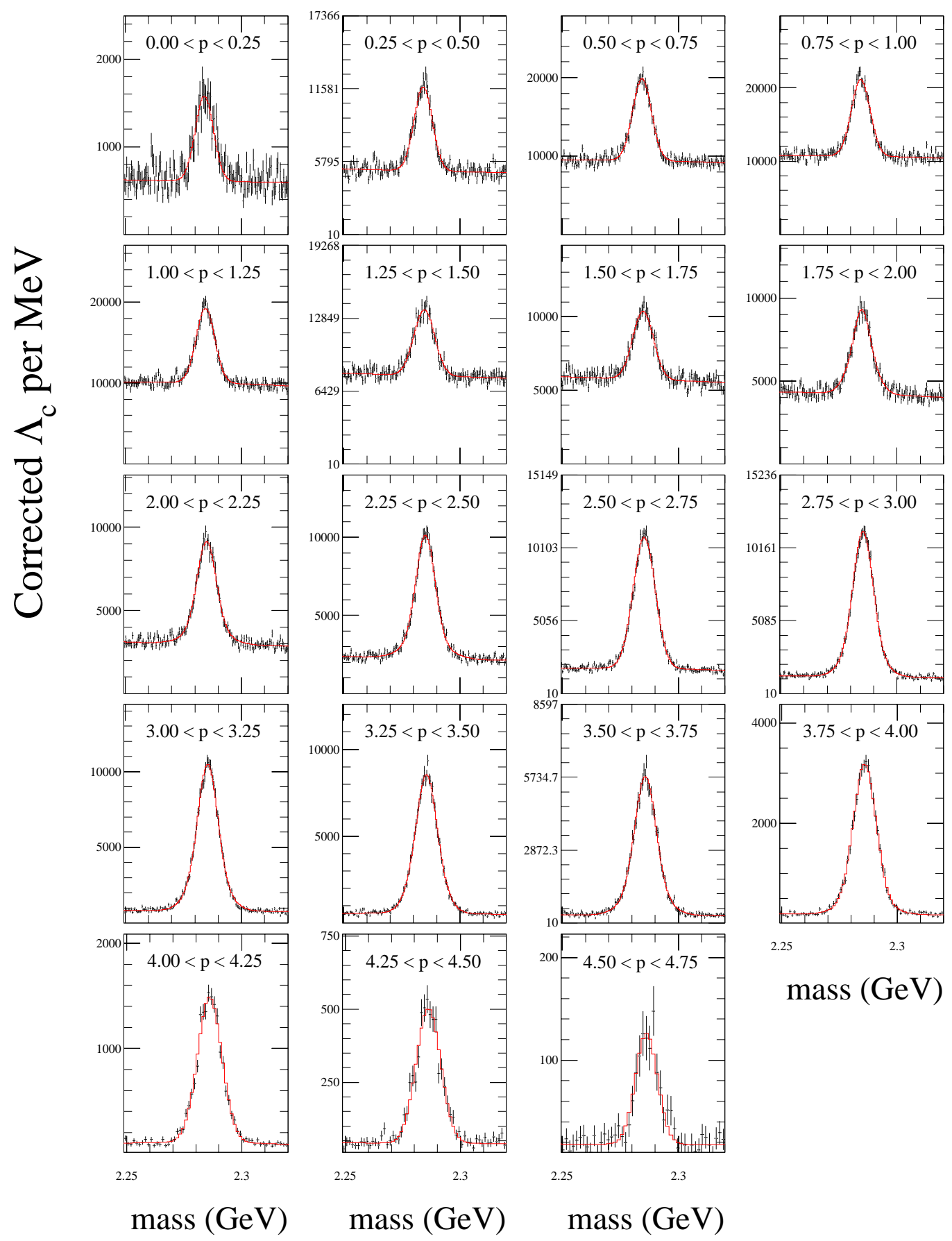

Figure 6.2: All data fits in 19 center of mass momentum bins 
statistics, the fits in the last 4 momentum bins are not very reliable. We would like to have more bins in the signal region in order to get a better fit, and fewer bins in the sidebands where we are statistically limited. The offpeak data is refit in figure 6.4 using variable size invariant mass bins with an equal number of $\Lambda_{c}$ candidates in each bin. This gives much better results in the last 4 momentum bins. We will use these fits for our spectrum measurement and use the fixed bin width fits as a cross check.

In order to study the effects of chosing a particular fitting function, the fits are redone three times with the following changes.

1) using the mass from each of the momentum slices in the AllData fits, as opposed to the linear fit in figure $6.1 \mathrm{~b}$

2) changing from linear to parabolic background and vice versa

3) increasing the number of entries in each invariant mass bin by about $50 \%$

The spectrum for each of the fitting functions is shown in figure 6.5. The largest deviation from the standard fit is taken as a systematic fit error.

\subsection{Systematic Errors}

\subsubsection{Reflections}

A reflection is an invariant mass peak that is created when either one of the daughter tracks is incorrectly identified, or when tracks that did not come from the same mother are combined. In order to look for reflections which might produce a fake $\Lambda_{c}$ signal, version 6.2 of the Pythia continuum event generator was used to generate 10 million events. The mass of all possible 3 charged track combinations was calculated, assigning the proton, pion, and kaon masses to each 

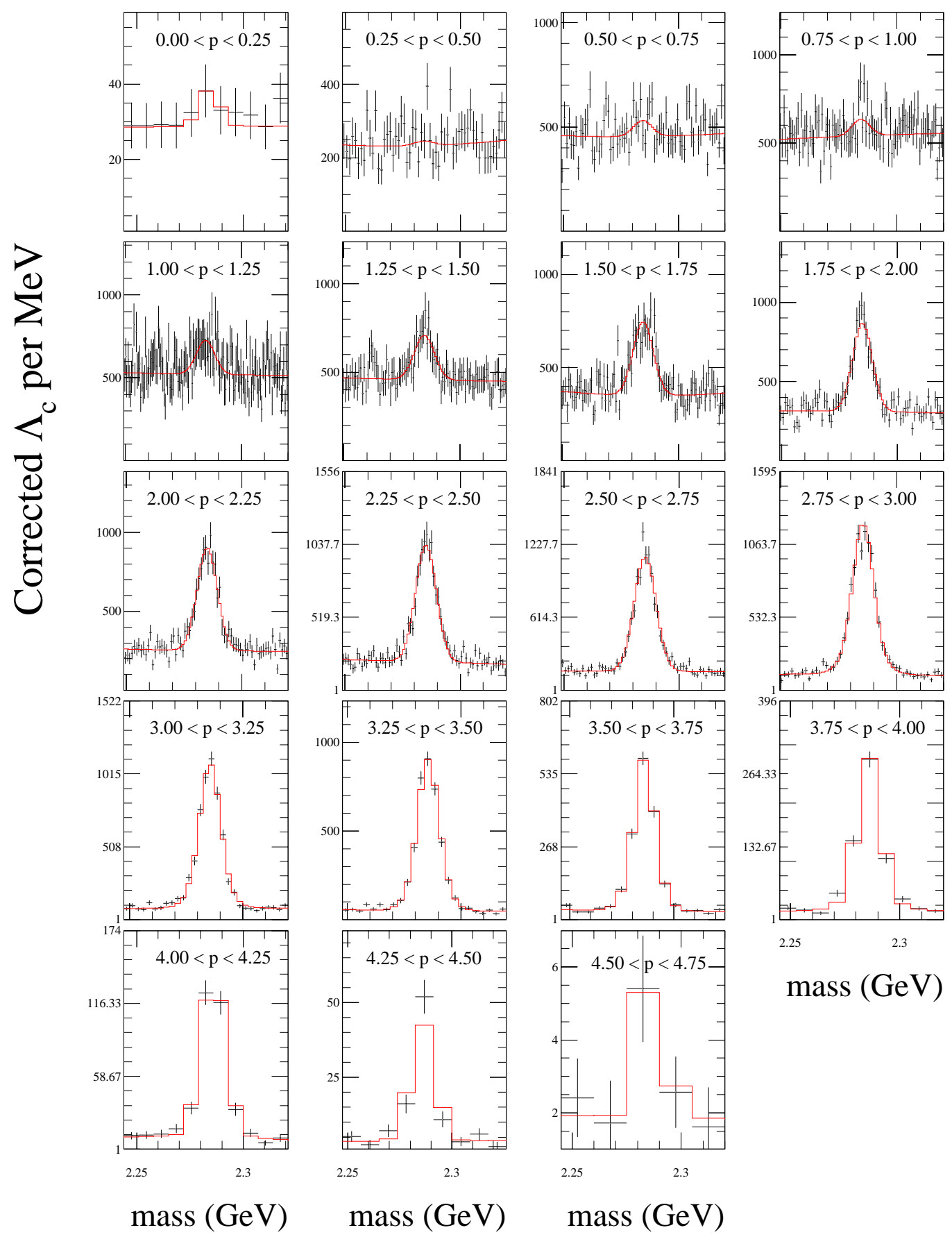

Figure 6.3: Offpeak data fits in 19 momentum bins. 

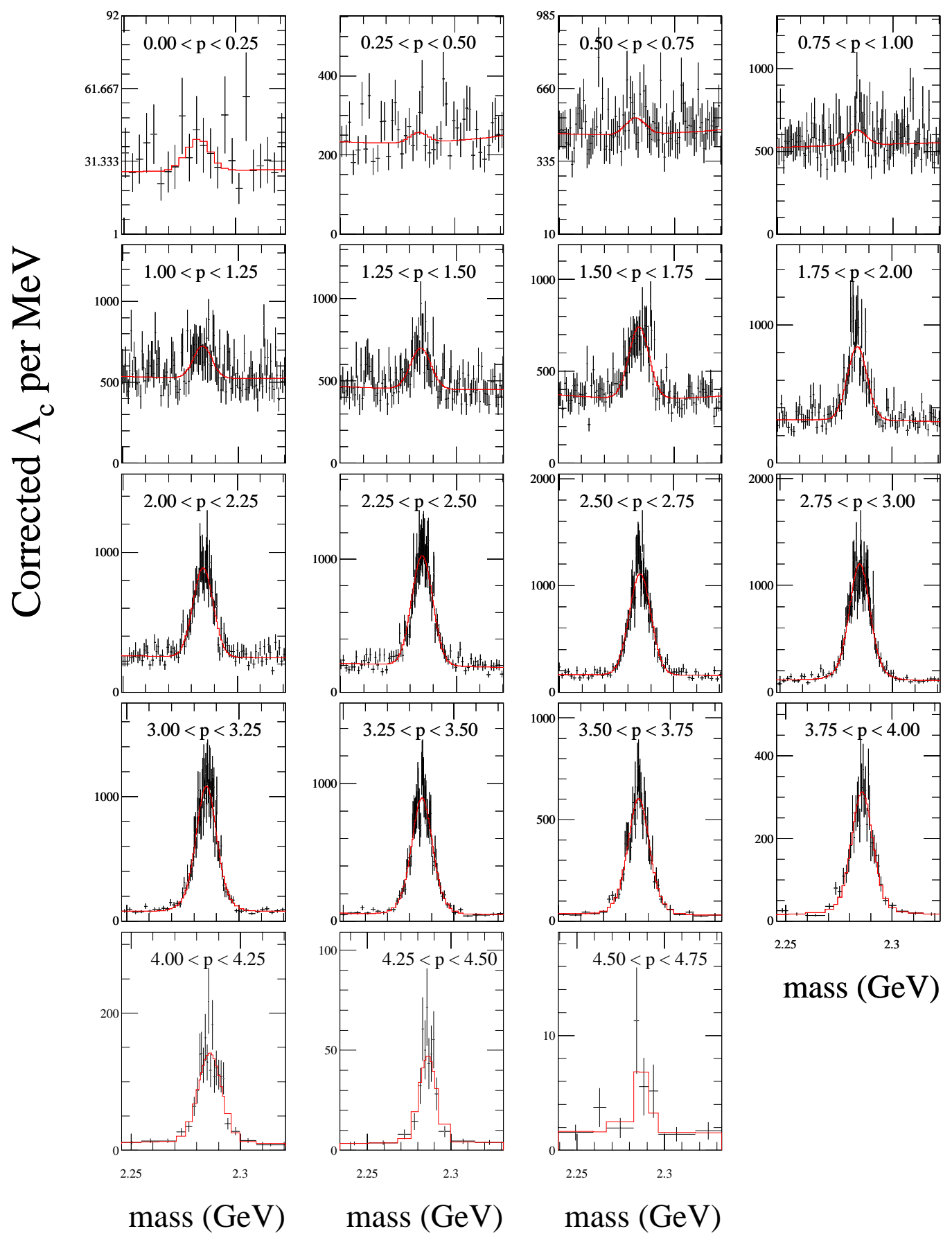

Figure 6.4: Offpeak data fits in 19 momentum bins using invariant mass bins of variable width 


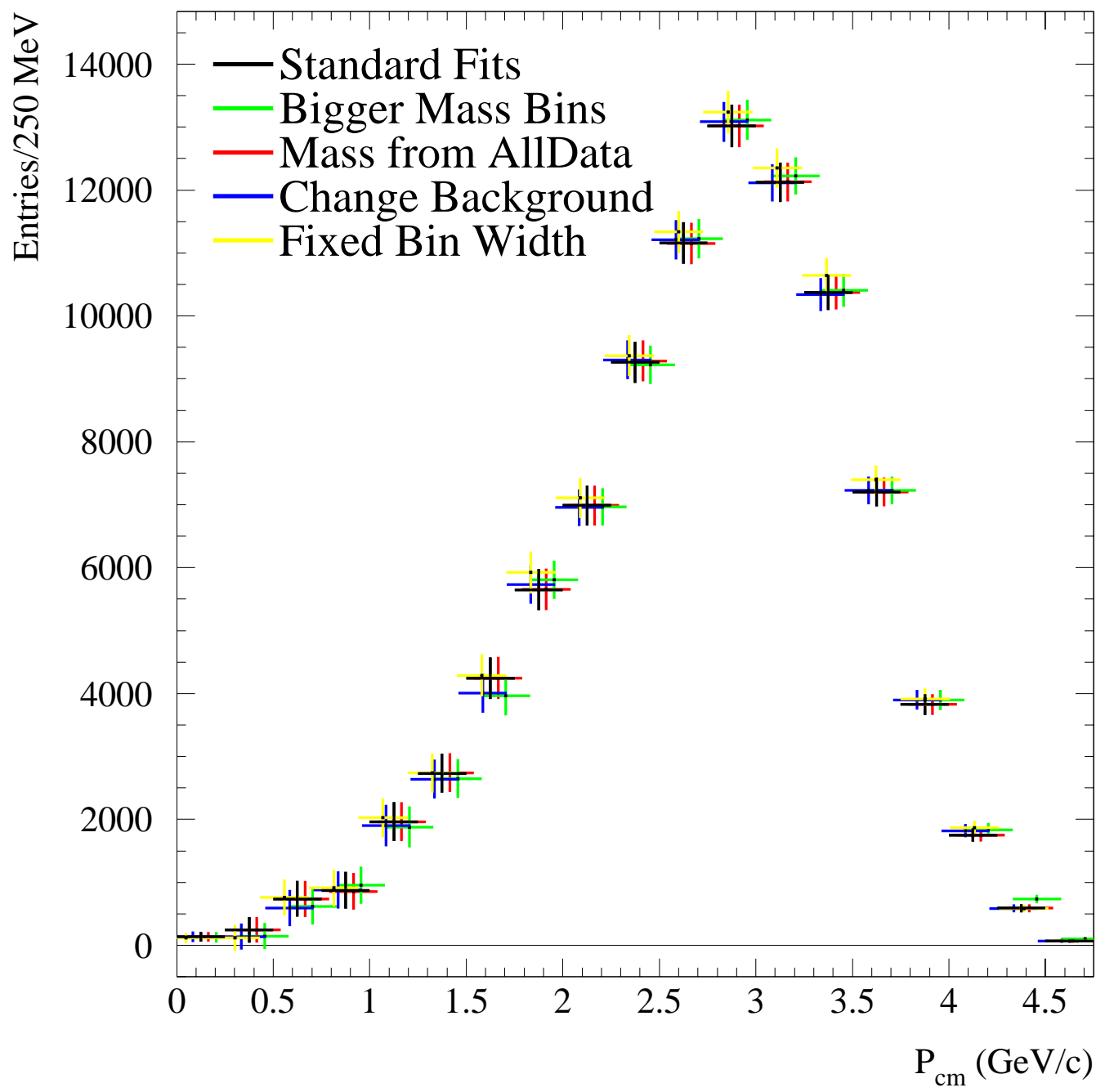

Figure 6.5: Continuum spectrum using five different fitting functions. The markers are slightly offset for easier viewing. 
of the tracks. Only combinations with less than two wrong mass assignments were kept. Because particle misidentification rates are less than 5\%, reflections resulting from two misidentifications will be about 20 times smaller and can be safely ignored.

Next, the number of entries within $10 \mathrm{MeV}$ of the $\Lambda_{c}$ mass were recorded for two types of decays; those in which all three charged tracks come from the same mother, and those in which two charged tracks have the same mother, and the mother of the mother particle is also the mother of the third charged track. The second case covers two body decays to a neutral and a charged particle with the subsequent decay of the neutral into two charged particles (example $D^{*} \rightarrow D^{0} \pi_{s}$ and $\left.D^{0} \rightarrow K \pi\right)$. The subscript $\mathrm{s}$ is used to distinguish pions decaying directly from the grandmother particle from those decaying from the neutral daughter. In these types of decays the 's' particle is usually slower.

All decays with more than 20 entires per 10 million events are shown in table 6.1. Because Pythia only produces about $60 \%$ as many $\Lambda_{c}$ 's as previous $10 \mathrm{GeV} / \mathrm{c}$ experiments, and also understates the $\pi K p$ branching fraction, the $\Lambda_{c} \rightarrow \pi K p$ yield was scaled up to its PDG value of .005 per event. All other rates are based on the Pythia default settings.

Because most reflections will result in a wide mass distribution that will be easily distinguishable from the signal, what we really want to know is how the number of entries in table 6.1 compares with the number of reflections showing up in the $\Lambda_{c}$ sidebands. This is what is shown in figure 6.6, which is the sideband subtracted reflection signal versus the $\Lambda_{c}$ candidate center of mass momentum. The true $\Lambda_{c}$ signal is represented by the triangles in each plot. The sidebands are defined as the regions 30 to $40 \mathrm{MeV} / \mathrm{c}$ higher or lower than the $\Lambda_{c}$ mass. The reflections are scaled up by a factor of 25 , in order to make them visible on the 


\begin{tabular}{|l|c|c|c|}
\hline Decay & & Misidentification & Number \\
\hline$\Lambda_{c} \rightarrow \pi K p$ & & none & 50,000 \\
$D^{+} \rightarrow K \pi \pi$ & $\pi$ as p & 8,238 \\
$D^{+} \rightarrow K K \pi$ & & $\mathrm{K}$ as p & 309 \\
$D^{+} \rightarrow K^{* 0} \pi_{s}$ & $K^{* 0} \rightarrow K \pi$ & $\pi$ as p & 320 \\
$D^{+} \rightarrow K^{* 0} \pi_{s}$ & $K^{* 0} \rightarrow K \pi$ & $\pi_{s}$ as p & 705 \\
$D_{0} \rightarrow K \pi \pi+X$ & & $\pi$ as p & 7,509 \\
$D_{0} \rightarrow K^{* 0} \pi_{s}+X$ & $K^{* 0} \rightarrow K \pi$ & $\pi_{s}$ as p & 751 \\
$D_{0} \rightarrow K^{* 0} \pi_{s}+X$ & $K^{* 0} \rightarrow K \pi$ & $\pi$ as p & 766 \\
$D^{*} \rightarrow D^{0} \pi_{s}$ & $D^{0} \rightarrow K \pi$ & $\pi$ as p & 3,350 \\
$D^{*} \rightarrow D^{0} \pi_{s}$ & $D^{0} \rightarrow K \pi$ & $\pi_{s}$ as p & 3,761 \\
$D_{s} \rightarrow K K \pi$ & & $\mathrm{K}$ as p & 490 \\
$D_{s} \rightarrow K^{* 0} \pi_{s}$ & $K^{* 0} \rightarrow K \pi$ & $\pi_{s}$ as p & 688 \\
$D_{s} \rightarrow \phi \pi$ & $\phi \rightarrow K K$ & $\mathrm{~K}$ as p & 289 \\
\hline
\end{tabular}

Table 6.1: Number of entries within $10 \mathrm{MeV}$ of the $\Lambda_{c}$ mass per 10 million generated continuum events

same plot, and assume $100 \%$ misidentification of one of the particles, so the true yields are expected to be about at about 500 times smaller than what is shown.

The only reflection coming close to $1 \%$ of the $\Lambda_{c}$ signal is the $D^{*+} \rightarrow D^{0} \pi_{s}$, $D^{0} \rightarrow K \pi$, where the $\pi$ is identified as $\mathrm{p}$, in the momentum bin $4.50 \leq p \leq$ $4.75 \mathrm{GeV} / \mathrm{c}$. This is the second to last momentum bin in figure 6.6. The last bin is above the $\Lambda_{c}$ kinematic limit. This momentum bin is shown plotted vs. invariant mass in figure 6.7. This figure assumes a $5 \%$ misidentification rate and so corresponds to what we expect to see in the data. The invariant mass is smeared with a Gaussian of width $5 \mathrm{MeV}$ in order to simulate detector resolution. 


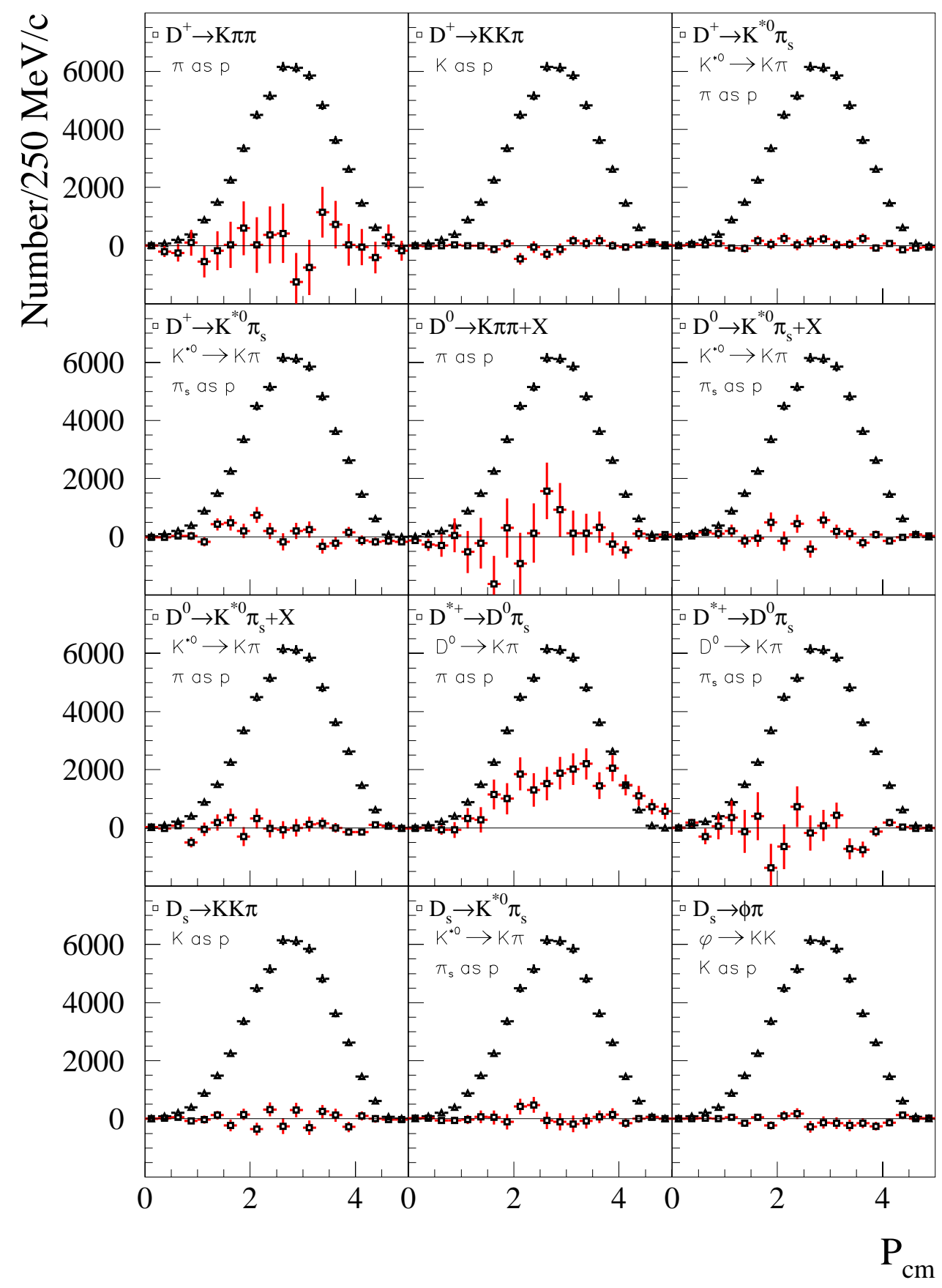

Figure 6.6: Generator level reflections (squares) compared with $\Lambda_{c}$ signal (triangles). Reflections are scaled up by a factor of 25 (to make them visible on the plot) and assume 100\% misidentification, so the actual rates should be at least 500 times smaller. 


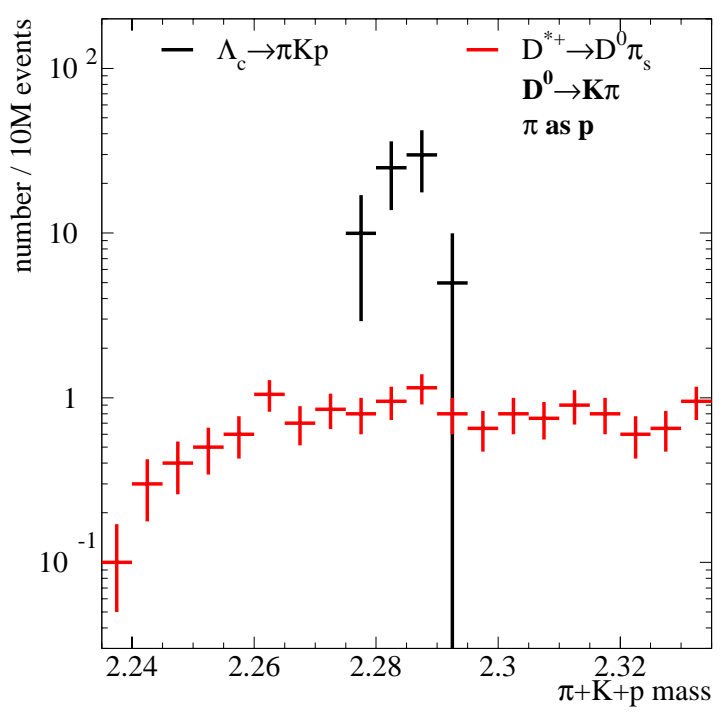

Figure 6.7: The $D^{*+}$ reflection assuming 5\% missID compared to the $\Lambda_{c}$ signal in the center of mass momentum bin $4.50 \leq p \leq 4.75 \mathrm{GeV} / \mathrm{c}$.

It is clear that the $D^{*+}$ reflection is flat across the $\Lambda_{c}$ mass range, and that the sideband subtracted signal was a result of the dropoff around $2.24 \mathrm{GeV}$. The reflection is also about 25 times smaller than the $\Lambda_{c}$ signal, so we do not have to worry about it.

One might be surprised that none of the decays of heavy charmed baryons showed up in figure 6.6. Figure 6.8 shows the reflection $\Sigma_{c}^{++} \rightarrow \pi_{s}^{+} \Lambda_{c}^{+}$where the $\pi_{s}$ and the pion from the $\Lambda_{c}$ decay are swapped, integrated over all momenta. The number of entries are scaled to match the PDG multiplicities. The invariant mass of the reflection is almost always less than the $\Lambda_{c}$ mass. 


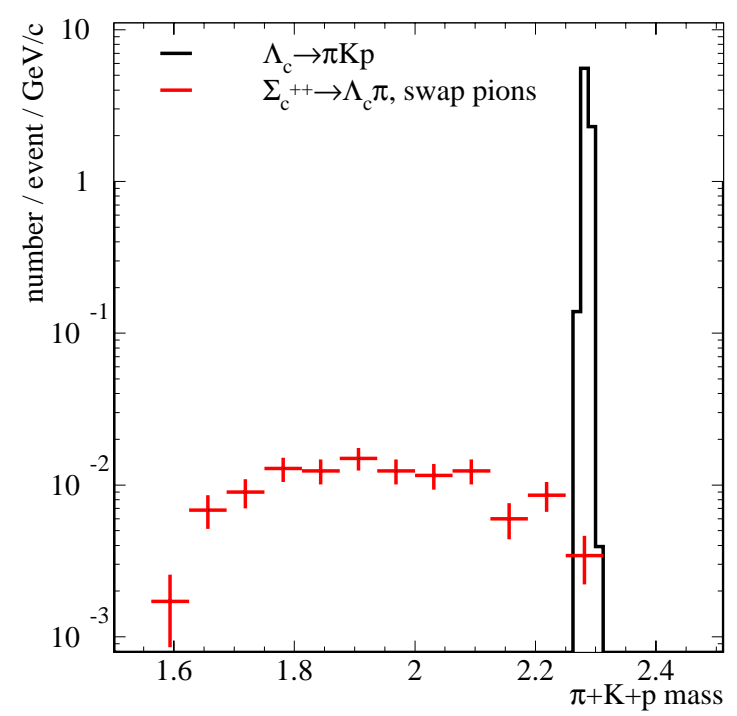

Figure 6.8: Reflection of $\Sigma_{c}^{++} \rightarrow \pi^{+} \Lambda_{c}^{+}$where the pions are swapped

\subsubsection{Momentum Resolution and Shift}

Because the detector has a non-zero momentum resolution, some of the reconstructed $\Lambda_{c}$ will diffuse into neighboring center of mass momentum bins, distorting the spectrum. This problem becomes worse as the difference in the number of entries between adjacent bins increases. Figure 6.9a shows the difference in true and reconstructed momenta for three center of mass momentum regions. In addition to smearing the momentum by a few $\mathrm{MeV}$, the reconstruction also shifts it slightly lower. This is probably due to the incorrect modeling of material interactions, which also caused the slope in the mass vs. momentum plot in figure 6.1b. The relative number of particles migrating up and down from each momentum bin is shown in figure $6.9 \mathrm{~b}$. Because of the momentum shift, a larger percentage of particles are migrating towards lower momentum bins. At high momentum, the momentum shift is less, so we might expect the number of particles migrat- 

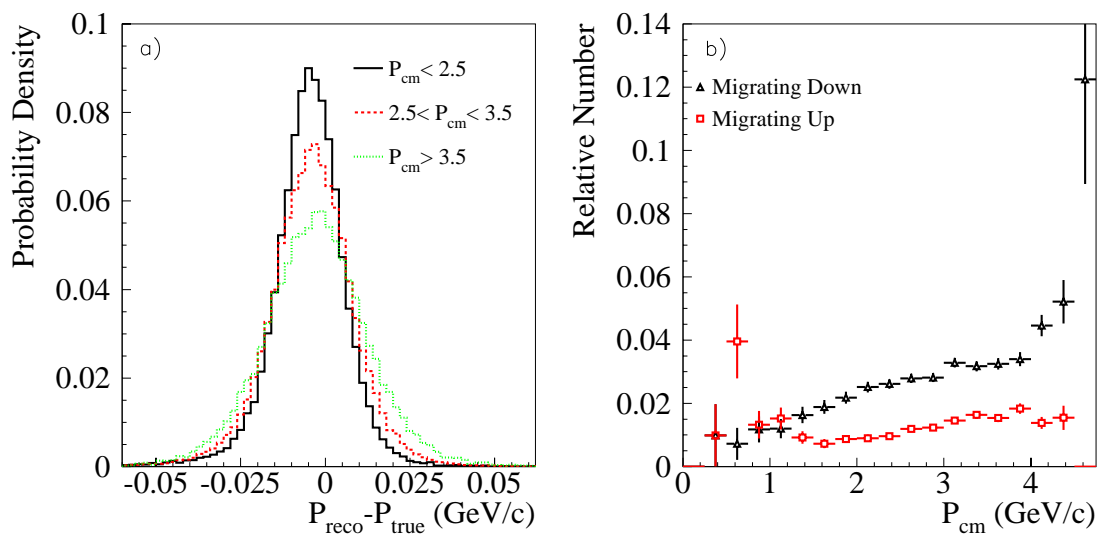

Figure 6.9: a) Momentum resolution for 3 different momentum ranges. b) Relative number of particles migrating to adjacent momentum bins.

ing up and down to be about the same. However, since the spectrum is sloped downwards at high momentum, a larger number of particles are near the low momentum edge of each bin, and these are more likely to migrate into the next bin.

Because the efficiency for finding a $\Lambda_{c}$ with reconstruted momentum $\mathrm{x}$ is defined as

$$
E=\frac{\text { number of particles with reconstructed momentum } \mathrm{x}}{\text { number of particles with true momentum } \mathrm{x}}
$$

this correction is already built into the efficiency map, if the Monte Carlo and data momentum spectra are the same. In order to cover any data/MC differences, the error on this correction will be defined as one third of the correction based on figure $6.9 \mathrm{~b}$. The size of the error can be seen in relation to all the other sources of error in figure 6.16 . 


\subsubsection{Resonant Substructure of Decays}

In the standard BaBar Monte Carlo the momentum vectors of the $\Lambda_{c}$ daughters are distributed evenly throughout the available phase space, but in reality, the $\pi K p$ mode has a resonant substructure. Decays passing through an intermediate resonant state, for example, a $K^{*}$, will result in a different daughter momentum vector distribution than in the non-resonant decays. Our primary concern is that the resonant states might have a different reconstruction efficiency, resulting in a bias in the efficiency calcualtion. The top three plots of figure 6.10 show the invariant masses of each two-particle combination in $\Lambda_{c} \rightarrow \pi K p$. The black curves show the Monte Carlo and the red curves are sideband subtracted data for $P_{c m} \geq 3$. The red line is scaled so that it fits under the black line. We can see that the structure is different in the data. The most obvious resonances are the $K_{892}^{*}$ in $\pi+K$, the $\Delta_{1232}$ in $\pi+p$ and the $\Lambda_{1520}$ in $K+p$

The bottom three plots show the efficiencies in bins of invariant mass for different values of $\Lambda_{c}$ center of mass momentum. The efficiency varies linearly except for a drop off at low $\pi+p$ mass and high $K+p$ mass. The efficiency increases with momentum, but because the shapes of the efficiency curves do not seem to change very much as momentum increases, we can do one error estimation, integrating over all $\Lambda_{c}$ momenta. Efficiency tables are made as a function of invariant mass for each of the three combinations, and these tables are then used to calculate the average Monte Carlo efficiency. The results are shown in table 6.2. Then, Monte Carlo events are killed off in order to make the invariant mass distributions match those seen in the data. The average efficiency is then recalculated. The changes for the efficiency in the $\pi+p, \pi+K$ and $K+p$ projections are $0.2 \%, 0.9 \%$ and $-0.3 \%$ respectively. We take the largest deviation, $0.9 \%$, as a systematic error. 

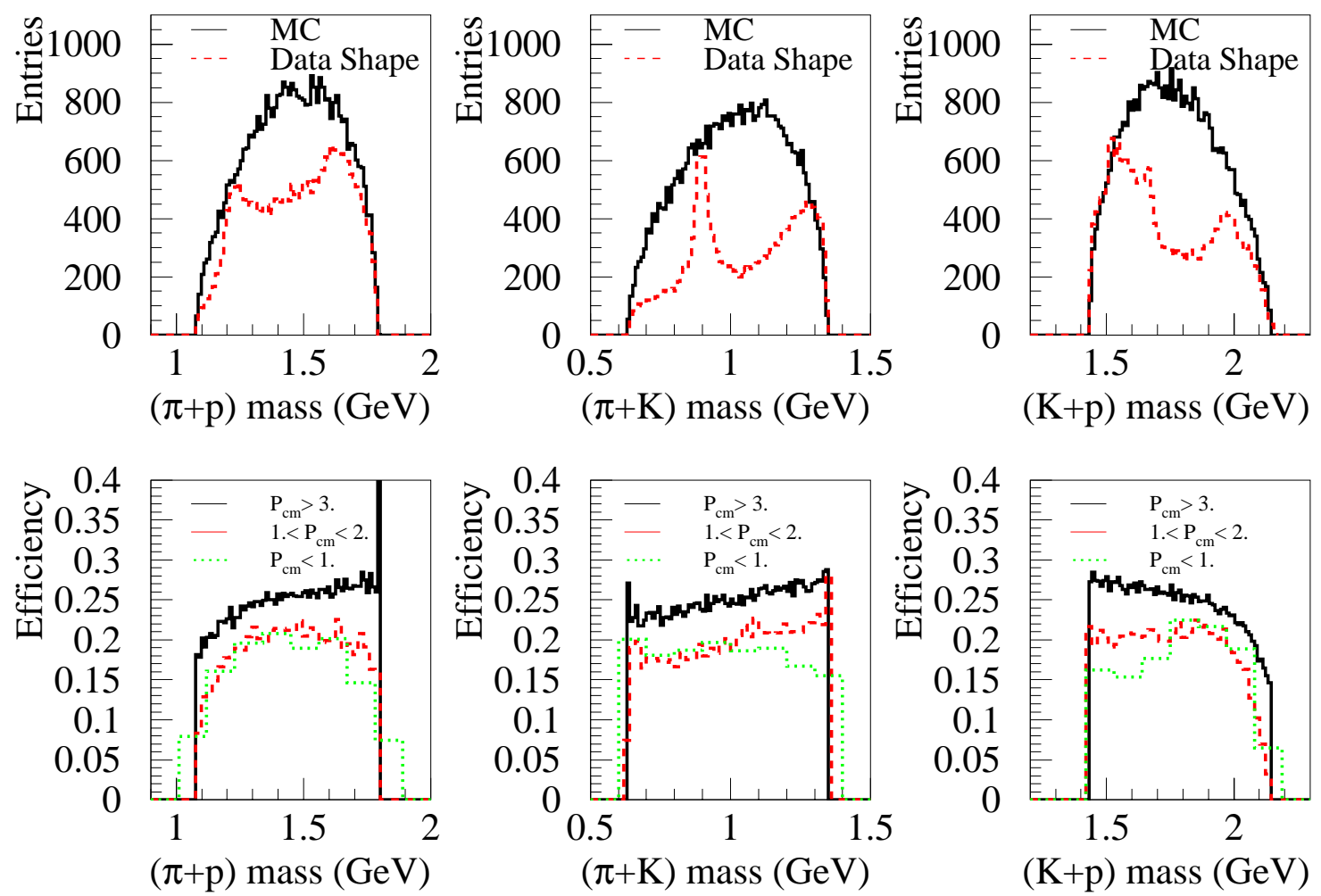

Figure 6.10: Top - Number of $\Lambda_{c}$ vs. invariant mass of any two of the daughters. Bottom - Reconstruction efficiency as a function of these invariant masses. 


\begin{tabular}{|c|c|c|c|}
\hline $\begin{array}{c}\text { Invariant } \\
\text { Mass }\end{array}$ & $\begin{array}{c}\text { Original } \\
\text { Efficiency }\end{array}$ & $\begin{array}{c}\text { Efficiency with } \\
\text { Resonances }\end{array}$ & Difference \\
\hline$\pi+p$ & .24170 & .24213 & $0.2 \%$ \\
$\pi+K$ & .24173 & .24386 & $0.9 \%$ \\
$K+p$ & .24169 & .24098 & $-0.3 \%$ \\
\hline
\end{tabular}

Table 6.2: Effect of resonant substructure on Monte Carlo Efficiency

\subsubsection{Proton Helicity Angle}

Because $\Lambda_{c} \rightarrow \pi K p$ is a weak decay, it is possible that the data would show a preferential direction for the daughter proton momentum in the $\Lambda_{c}$ rest frame. Because the Monte Carlo does not include this effect, our efficiency calculation could be biased by its appearance in the data. Figure 6.11 shows the cosine of the angle between the proton and $\Lambda_{c}$ momenta in the $\Lambda_{c}$ rest frame. Because fast $\Lambda_{c}$ are more likely to retain the helicity of the original c quark, any effect should be more noticable at high momentum. The plot shows efficiency corrected, sideband subtracted $\Lambda_{c}$ above $3.5 \mathrm{GeV} / \mathrm{c}$ in the center of mass frame. The line is fit to the data, but is also consistant with Monte Carlo, which has been scaled to have the same area as the data. The downward slope suggests that decays with a backwards moving $K$ and $\pi$ are reconstructed with lower efficiency, but, because the effect is the same in Monte Carlo and data, we do not need to make a correction.

\subsubsection{MC/Data Momentum Spectrum Shift}

If the Monte Carlo momentum spectrum is shifted with respect to the data, this can also cause a bias in the efficiency calculation. In order to measure 


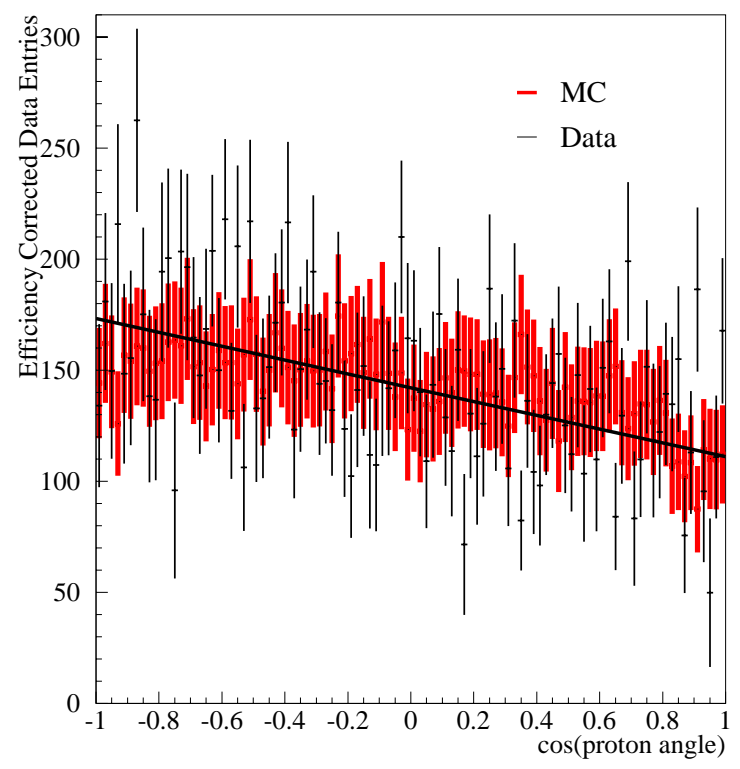

Figure 6.11: Proton helicity angle

the size of this bias, we could create a new set of Monte Carlo with a shifted spectrum, and then run it through the detector simulation and make a new efficiency map. However, since this would take a large amount of computer time, we will substitute our calculated efficiency map and a random number generator for the detector simulation, and feed in shifted $\Lambda_{c}$ spectra to make new efficiency maps. We will then correct the original Monte Carlo using the new maps, and see how much the $\Lambda_{c}$ yield changes. Since we are using signal Monte Carlo there is almost no background, and we can get the $\Lambda_{c}$ yield simply by subtracting an equal amount of sideband from the $50 \mathrm{MeV}$ width signal region.

Figure 6.12 shows the effect of shifting the input momentum spectrum up or down by a half or a quarter of a bin. Because of lower statistics, it is more difficult to constrain the effect of this bias at low and high momenta. The error is taken to be the half-width of the yellow band. 


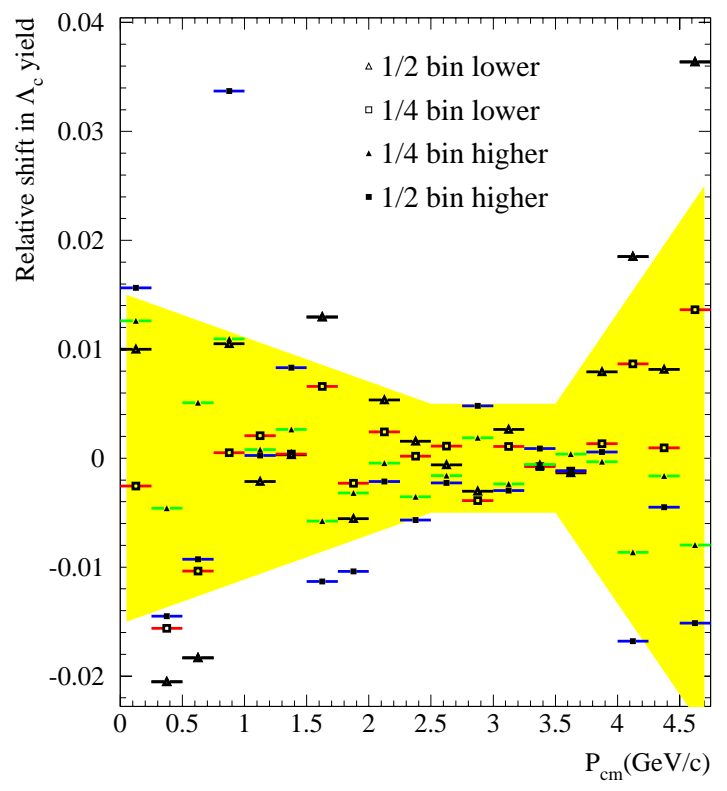

Figure 6.12: Effect of shifting the momentum distribution used to make the efficiency map on $\Lambda_{c}$ yield

\subsubsection{Angular Distribution in Monte Carlo and Data}

As described in section 5.2.1, we must extrapolate the $\Lambda_{c}$ yield into regions of low reconstruction efficiency using the Monte Carlo $\Lambda_{c}$ angular distribution. In order to test this distribution against the data, we divide it into 6 regions of center of mass polar angle. We extrapolate each slice using the Monte Carlo and then compare the result to that obtained from our standard measurement, which integrates over all six angular bins. A MC/data difference in the number of $\Lambda_{c}$ in one of the angular slices would cause this extrapolation to be inconsistant with the others. Problems with the efficiency corrections would also show up as an inconsistancy.

Because we have lower statistics for each angular slice, we fit the invariant mass distribution of each momentum bin with just a single Gaussian with a 
linear background. The momentum bins below $1 \mathrm{GeV} / \mathrm{c}$ and above $4.25 \mathrm{GeV} / \mathrm{c}$ do not have enough statistics to reveal a peak near the $\Lambda_{c}$ mass and are not used. In order to make the fits work below $1.5 \mathrm{GeV} / \mathrm{c}$, the center of the Gaussian is required to be within $30 \mathrm{MeV}$ of the $\Lambda_{c}$ mass in this region.

The results are shown in figure 6.13a. Only statistical errors are shown, but, since each center of mass angular bin corresponds to a different detector region, some of the systematic errors should be uncorreleated as well. Therefore, the errors in the plots are underestimated. To test the consistancy of the 6 measurements we minimise the $\chi^{2}$ for each momentum bin, and plot $\chi^{2} / D o F$ for 5 degrees of freedom. In figure $6.13 \mathrm{~b} \chi^{2} / D o F$ is shown for each bin and for the bins from $1.00 \leq P_{c m} \leq 4.25$ combined. The error bars are such that we expect only $16 \%$ of the points to be above one by a distance larger than the error. The $2.25 \leq P_{c m} \leq 2.50$ has a large $\chi^{2}$ but we have no reason to believe that this is not a statistical fluctuation. The total $\chi^{2}$ is consistant, even without including the systematic errors.

In order to look for changes in the cross section, or shifts in the peak of the spectrum, we fit a Gaussian to each angle bin spectrum between 1.50 and 4.25 GeV/c. The normalisation and peak location of the 6 Gaussians are shown in figures $6.13 \mathrm{c} \& d$. If the center of mass angular distribution of $\Lambda_{c}$ is $1+$ $a(p) \cos ^{2} \theta_{c m}$, the probability density should be independent of 'a' at $\cos \theta=$ $\pm \sqrt{\frac{1}{3}}=.577$. This turns out to be at the center of our second (red) angular bin. Thus, if the Monte Carlo used an incorrect value for 'a', we would expect the extrapolation to still be correct in the second bin, and the variation in bin one to be compensated by the highest four bins. Since the second bin is the lowest point this is not the case, and we have no evidence that the Monte Carlo has incorrectly modelled the angular distribution. 
Figure $6.13 \mathrm{~d}$, shows that the locations of the peaks of the 6 spectra are consistant. If we were to add another angular bin for the region $0.3 \leq \cos \theta \leq 0.6$ we would see that its spectrum was shifted towards higher momenta. Looking back at figure 5.4, we see that this extra bin would correspond to the region just above the black line, where the efficiency is quickly varying and thus difficult to calculate.

\subsubsection{Time Dependance}

Because detector conditions change over time it is important to check that there are no systematic effects which lead to changes in the calculated spectrum. Our efficiency calculations and data/MC corrections were made to be independent of time in order to decrease the statistical error. We need to check that this time averaging has not created a bias in our results.

Figure $6.14 \mathrm{a}$ shows the shape of the spectrum over 6 time periods of roughly equal luminosity. The errors are statistical only. The most significant change over the period of data taking was the voltage on the drift chamber. For time slice one it was equal to $1900 \mathrm{~V}$. For time two it was $1960 \mathrm{~V}$. For later times it was 1930V. There does not appear to be a change in the shape of the spectrum.

Figure 6.14 b shows a "cross section" for each time period, but because the different angular distribution of the B decays has not yet been taken into account, this is not the true cross section. It looks like the first time period may be lower in the data. The Monte Carlo, which tries to mimic the detector conditions on a month to month basis, does not drop as much in the first time slice, but, because the difference is not very significant statistically, we have decided not to add an additional normalization error. 

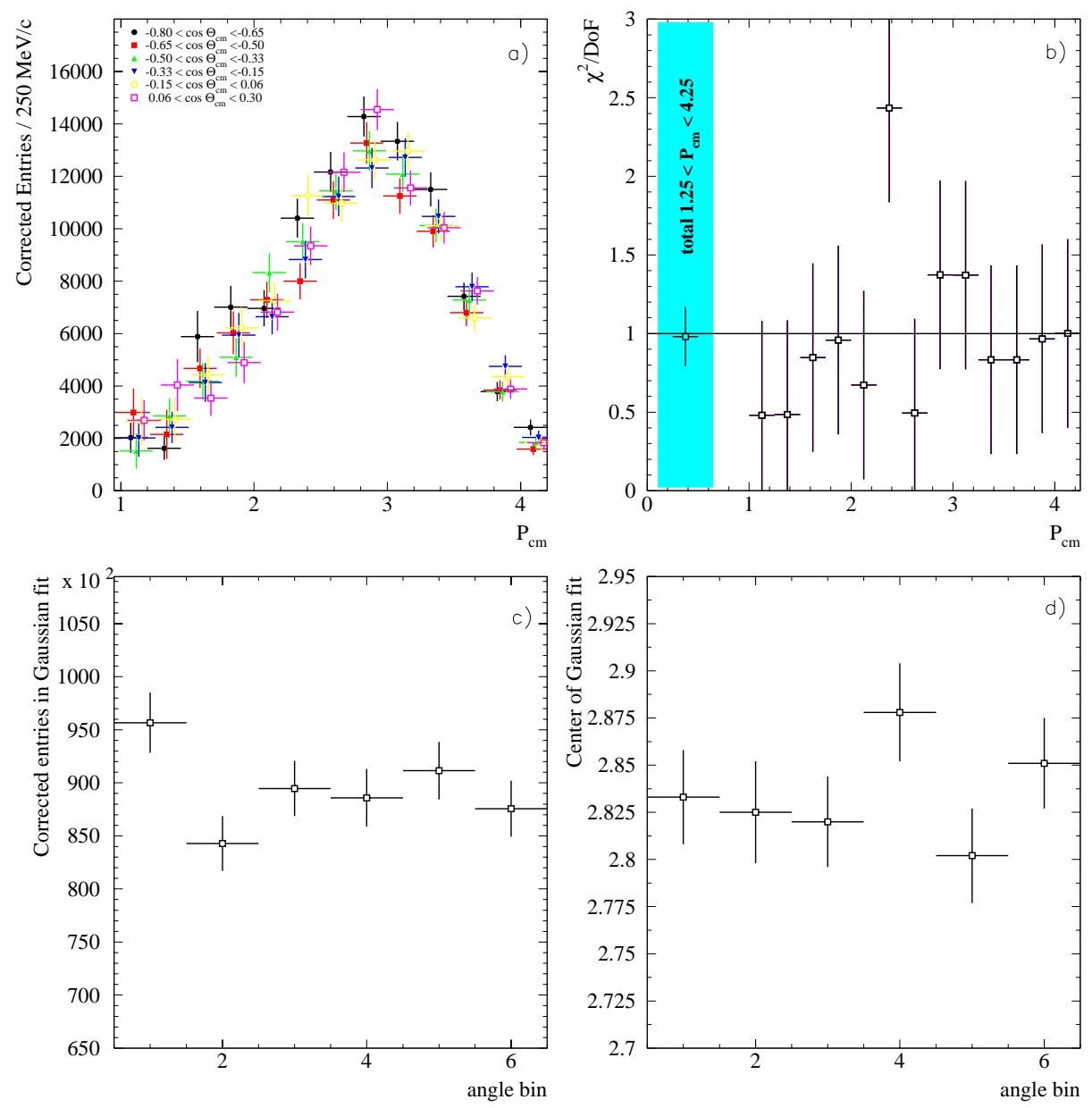

Figure 6.13: a) Comparison of efficiency corrected spectra from six different center of mass angle regions. b) the $\chi^{2} / D o F$ for each momentum bin in a). c) Number of entries in a Gaussian fit from $1.5 \leq P_{c m} \leq 4.25$ for each angle bin. d) Center of the Gaussian fits. 

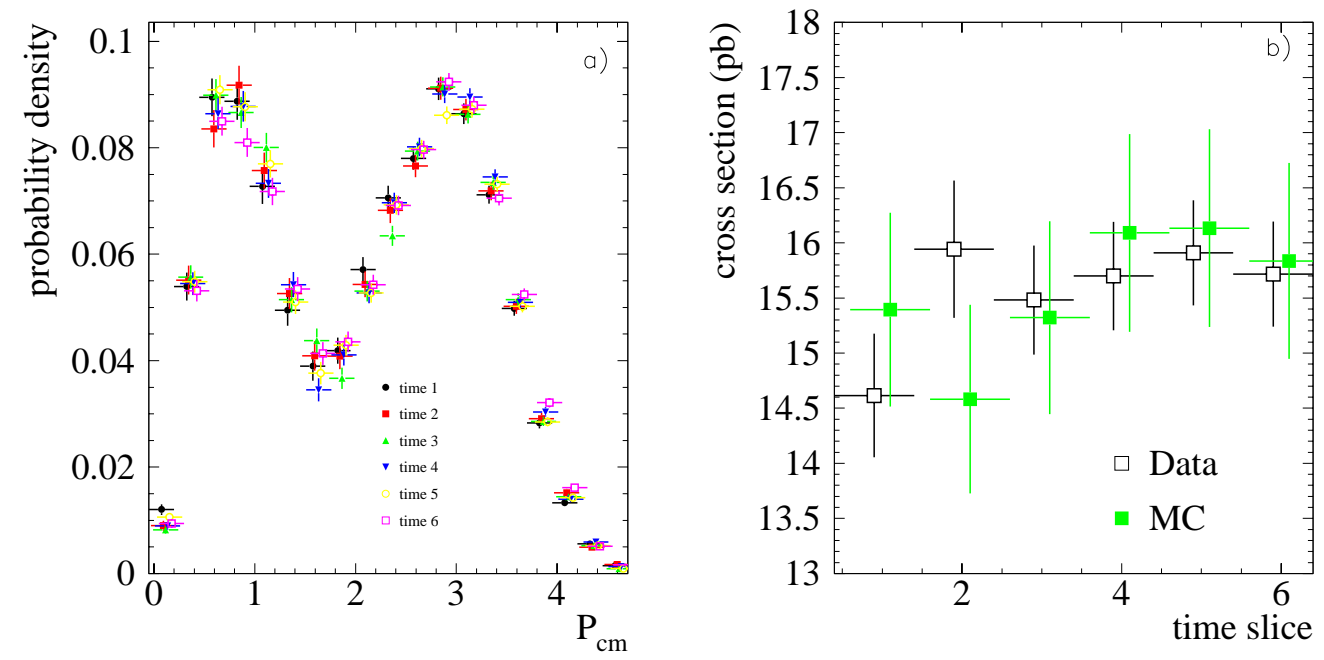

Figure 6.14: Spectrum time dependance. a) shows the shape of the spectrum over 6 time periods. b) shows a measure of cross section vs. time.

\subsection{Results}

The final section of this chapter contains tables of all of the previously mentioned errors and displays our final result. Comparisons with previous experiments and with the various hadronization models and fragmetation functions will be made in the next chapter.

Figure 6.15 shows our final result, the momentum spectrum of $\Lambda_{c} \rightarrow \pi K p$ at $10.54 \mathrm{GeV}$. Because of the large uncertainty of the branching fraction of $(5.0 \pm$ $1.3) \%$, we have chosen to plot the cross section for this particular mode only.

The measured spectum is very smooth. None of the individual points appear to deviate from a reasonable interpolation of the nearest neighbors by more than the reported errors.

The various errors are shown in figure 6.16 and in the accompanying tables. 


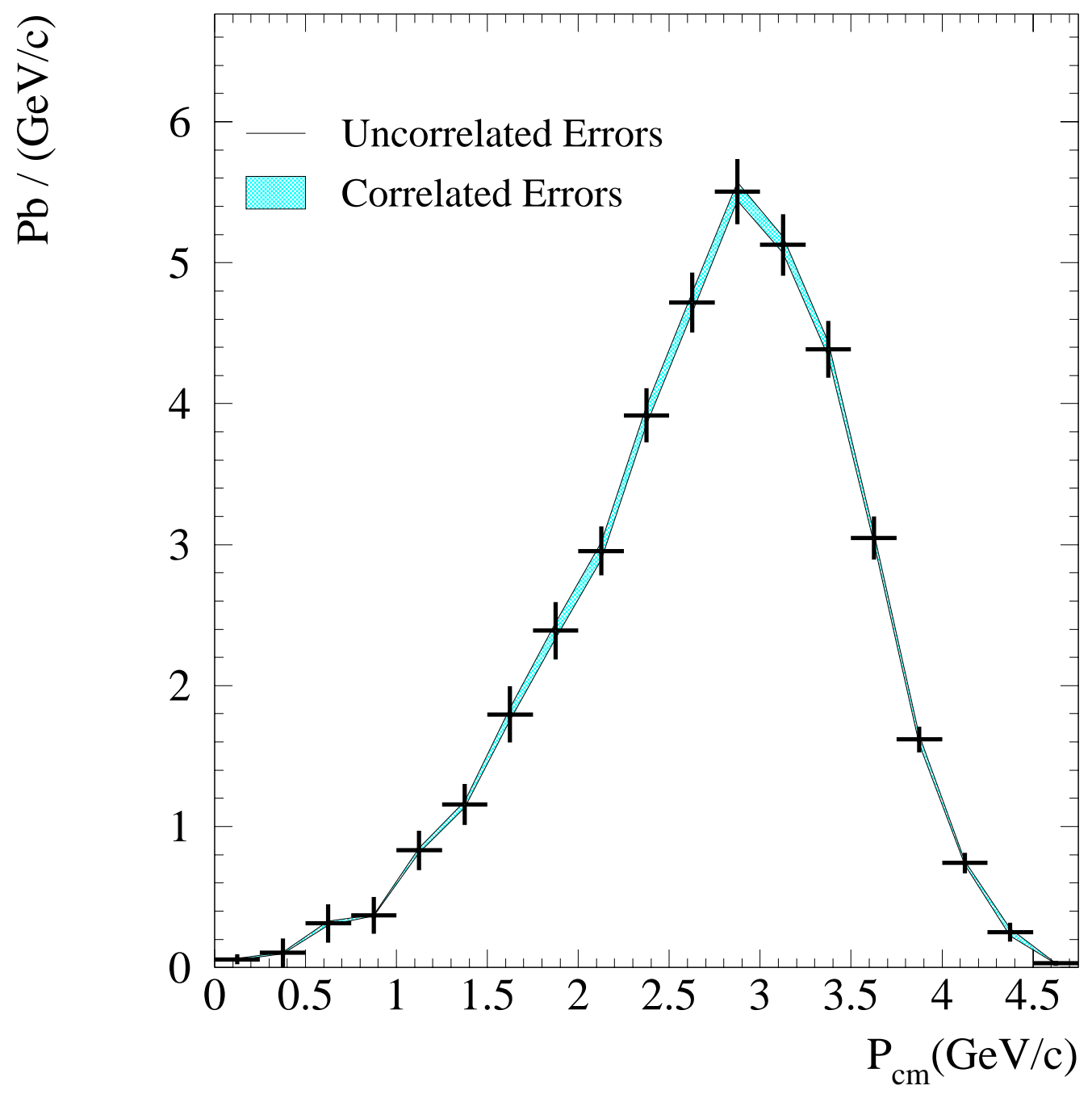

Figure 6.15: The continuum spectrum for $\Lambda_{c} \rightarrow \pi K p$. The error bars show the independant and statistical errors. The shaded region shows the square root of the diagonal terms of the correlated error matrix. 


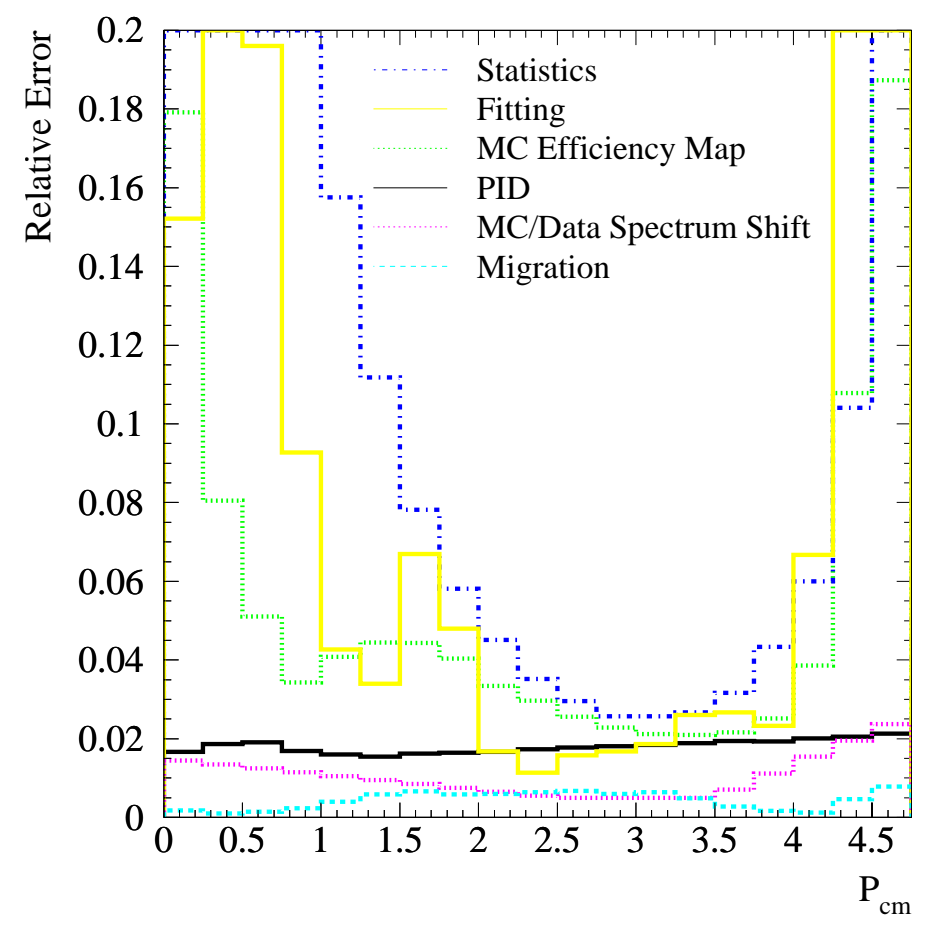

Figure 6.16: Relative errors on continuum measurement

Not shown are the $0.9 \%$ error caused by the data/MC difference in Dalitz structure, the $1 \%$ error on the BaBar integrated luminosity measurement, and the $2.48 \%$ tracking error, which are the same in every bin.

We need to address the issue of error correlations between momentum bins. We classify each error as being one of three types.

Normalization errors are those which are perfectly correlated and the same relative amount in each momentum bin. These errors do not have any effect on the shape of the spectrum, and can be ignored except when calculating the total rate. The BaBar luminosity error of $1 \%$ is a normalization error. The tracking error, which is expected to be perfectly correlated, averages $2.48 \%$ with a maximum deviation of $0.03 \%$ can also be considered a normalization error. This 
gives us a total normalization error of $2.67 \%$.

Independent errors are those which have no correlation between momentum bins. The statistical and fitting errors are completely independent. Particle identification errors have many different correlations, but since none of them dominate, we can treat the sum as being independent. The Dalitz, migration and $\mathrm{MC} /$ data shift errors are all less than 1.5\%, and any correlations would have little effect on our result, so they can also be treated as independent.

This leaves the MC efficiency map error, which turns out to be correlated. As described in section 5.2.1, the statistical error on the $\mathrm{MC}$ efficiency could be reduced in regions of detector space with a small efficiency gradient by integrating the efficiency over a large area. The result of this was a covariance of the efficiency between adjacent bins of around 25\%. The highest and lowest momentum bins have covariances extending further, to the forth nearest neighbor, because of the increased radius of integration used in these low statistic regions. The $\mathrm{MC}$ efficiency matrix can be factored into independent and correlated pieces, which are shown in tables 6.3 and 6.5. The shaded region in figure 6.15 is the square root of the diagonal terms of the error matrix.

The three largest errors - the statistical error on the fit, the error caused by the uncertianty of which function best fits the mass peaks, and the Monte Carlo efficiency map error — are all statistical in nature, implying that a future BaBar measurement using more luminosity could improve on our measurement, especially at low and high momenta. The BaBar integrated luminoisty could be increased by a factor of four by the end of 2008. So we can expect to be able to decrease the errors to roughly half of what they are now.

In the next chapter we will see how our measurement compares to the various hadronization models. 


\begin{tabular}{|c|c|c|c|c|c|c|c|c|c|c|c|c|c|c|c|c|c|c|c|}
\hline$P_{c m}$ & $\frac{1}{8}$ & $\frac{3}{8}$ & $\frac{5}{8}$ & $\frac{7}{8}$ & $1 \frac{1}{8}$ & $1 \frac{3}{8}$ & $1 \frac{5}{8}$ & $1 \frac{7}{8}$ & $2 \frac{1}{8}$ & $2 \frac{3}{8}$ & $2 \frac{5}{8}$ & $2 \frac{7}{8}$ & $3 \frac{1}{8}$ & $3 \frac{3}{8}$ & $3 \frac{5}{8}$ & $3 \frac{7}{8}$ & $4 \frac{1}{8}$ & $4 \frac{3}{8}$ & $4 \frac{5}{8}$ \\
\hline$\frac{1}{8}$ & 7.5 & 6.3 & 5.8 & 2.3 & 0.8 & 0.0 & 0.0 & 0.0 & 0.0 & 0.0 & 0.0 & 0.0 & 0.0 & 0.0 & 0.0 & 0.0 & 0.0 & 0.0 & 0.0 \\
\hline$\frac{3}{8}$ & 6.3 & 5.3 & 2.9 & 1.1 & 0.7 & 0.0 & 0.0 & 0.0 & 0.0 & 0.0 & 0.0 & 0.0 & 0.0 & 0.0 & 0.0 & 0.0 & 0.0 & 0.0 & 0.0 \\
\hline$\frac{5}{8}$ & 5.8 & 2.9 & 4.5 & 1.8 & 0.6 & 0.4 & 0.0 & 0.0 & 0.0 & 0.0 & 0.0 & 0.0 & 0.0 & 0.0 & 0.0 & 0.0 & 0.0 & 0.0 & 0.0 \\
\hline$\frac{7}{8}$ & 2.3 & 1.1 & 1.8 & 1.3 & 1.6 & 0.4 & 0.0 & 0.0 & 0.0 & 0.0 & 0.0 & 0.0 & 0.0 & 0.0 & 0.0 & 0.0 & 0.0 & 0.0 & 0.0 \\
\hline $1 \frac{1}{8}$ & 0.8 & 0.7 & 0.6 & 1.6 & 1.9 & 1.9 & 0.4 & 0.0 & 0.0 & 0.0 & 0.0 & 0.0 & 0.0 & 0.0 & 0.0 & 0.0 & 0.0 & 0.0 & 0.0 \\
\hline $1 \frac{3}{8}$ & 0.0 & 0.0 & 0.4 & 0.4 & 1.9 & 2.0 & 2.1 & 0.6 & 0.0 & 0.0 & 0.0 & 0.0 & 0.0 & 0.0 & 0.0 & 0.0 & 0.0 & 0.0 & 0.0 \\
\hline $1 \frac{5}{8}$ & 0.0 & 0.0 & 0.0 & 0.0 & 0.4 & 2.1 & 2.3 & 2.3 & 0.7 & 0.0 & 0.0 & 0.0 & 0.0 & 0.0 & 0.0 & 0.0 & 0.0 & 0.0 & 0.0 \\
\hline $1 \frac{7}{8}$ & 0.0 & 0.0 & 0.0 & 0.0 & 0.0 & 0.6 & 2.3 & 2.3 & 2.2 & 0.8 & 0.0 & 0.0 & 0.0 & 0.0 & 0.0 & 0.0 & 0.0 & 0.0 & 0.0 \\
\hline $2 \frac{1}{8}$ & 0.0 & 0.0 & 0.0 & 0.0 & 0.0 & 0.0 & 0.7 & 2.2 & 2.1 & 1.8 & 0.6 & 0.0 & 0.0 & 0.0 & 0.0 & 0.0 & 0.0 & 0.0 & 0.0 \\
\hline $2 \frac{3}{8}$ & 0.0 & 0.0 & 0.0 & 0.0 & 0.0 & 0.0 & 0.0 & 0.8 & 1.8 & 1.7 & 1.6 & 0.6 & 0.0 & 0.0 & 0.0 & 0.0 & 0.0 & 0.0 & 0.0 \\
\hline $2 \frac{5}{8}$ & 0.0 & 0.0 & 0.0 & 0.0 & 0.0 & 0.0 & 0.0 & 0.0 & 0.6 & 1.6 & 1.5 & 1.3 & 0.5 & 0.0 & 0.0 & 0.0 & 0.0 & 0.0 & 0.0 \\
\hline $2 \frac{7}{8}$ & 0.0 & 0.0 & 0.0 & 0.0 & 0.0 & 0.0 & 0.0 & 0.0 & 0.0 & 0.6 & 1.3 & 1.2 & 1.2 & 0.5 & 0.0 & 0.0 & 0.0 & 0.0 & 0.0 \\
\hline $3 \frac{1}{8}$ & 0.0 & 0.0 & 0.0 & 0.0 & 0.0 & 0.0 & 0.0 & 0.0 & 0.0 & 0.0 & 0.5 & 1.2 & 1.1 & 1.1 & 0.6 & 0.0 & 0.0 & 0.0 & 0.0 \\
\hline $3 \frac{3}{8}$ & 0.0 & 0.0 & 0.0 & 0.0 & 0.0 & 0.0 & 0.0 & 0.0 & 0.0 & 0.0 & 0.0 & 0.5 & 1.1 & 1.2 & 1.2 & 0.7 & 0.0 & 0.7 & 0.9 \\
\hline $3 \frac{5}{8}$ & 0.0 & 0.0 & 0.0 & 0.0 & 0.0 & 0.0 & 0.0 & 0.0 & 0.0 & 0.0 & 0.0 & 0.0 & 0.6 & 1.2 & 1.2 & 1.4 & 0.8 & 1.4 & 2.7 \\
\hline $3 \frac{7}{8}$ & 0.0 & 0.0 & 0.0 & 0.0 & 0.0 & 0.0 & 0.0 & 0.0 & 0.0 & 0.0 & 0.0 & 0.0 & 0.0 & 0.7 & 1.4 & 2.0 & 2.0 & 1.8 & 3.9 \\
\hline $4 \frac{1}{8}$ & 0.0 & 0.0 & 0.0 & 0.0 & 0.0 & 0.0 & 0.0 & 0.0 & 0.0 & 0.0 & 0.0 & 0.0 & 0.0 & 0.0 & 0.8 & 2.0 & 2.0 & 4.4 & 4.4 \\
\hline $4 \frac{3}{8}$ & 0.0 & 0.0 & 0.0 & 0.0 & 0.0 & 0.0 & 0.0 & 0.0 & 0.0 & 0.0 & 0.0 & 0.0 & 0.0 & 0.7 & 1.4 & 1.8 & 4.4 & 10.6 & 10.7 \\
\hline $4 \frac{5}{8}$ & 0.0 & 0.0 & 0.0 & 0.0 & 0.0 & 0.0 & 0.0 & 0.0 & 0.0 & 0.0 & 0.0 & 0.0 & 0.0 & 0.9 & 2.7 & 3.9 & 4.4 & 10.7 & 10.8 \\
\hline
\end{tabular}

Table 6.3: Error matrix for the correlated part of the MC efficiency error. The square root of each term is taken so that the percent error can be read off the diagonal 


\begin{tabular}{|c|c|c|c|c|c|}
\hline $\begin{array}{c}\text { Momentum } \\
\text { Range }\end{array}$ & $\begin{array}{c}\text { Spectrum } \\
\mathrm{pb} /(\mathrm{GeV} / \mathrm{c})\end{array}$ & $\begin{array}{c}\text { Total } \\
\text { Error }(\%)\end{array}$ & $\begin{array}{l}\text { Statistical } \\
\text { Error (\%) }\end{array}$ & $\begin{array}{c}\text { Independent } \\
\text { Error (\%) }\end{array}$ & $\begin{array}{l}\text { Correlated } \\
\text { Error }(\%)\end{array}$ \\
\hline $0.00-0.25$ & 0.058 & 58.92 & 54.61 & 20.83 & 7.48 \\
\hline $0.25-0.50$ & 0.104 & 98.98 & 83.97 & 52.13 & 5.32 \\
\hline $0.50-0.75$ & 0.313 & 43.73 & 38.75 & 19.75 & 4.53 \\
\hline $0.75-1.00$ & 0.369 & 35.05 & 33.59 & 9.92 & 1.31 \\
\hline $1.00-1.25$ & 0.831 & 16.92 & 15.75 & 5.88 & 1.92 \\
\hline $1.25-1.50$ & 1.157 & 12.65 & 11.18 & 5.58 & 1.97 \\
\hline $1.50-1.75$ & 1.785 & 11.38 & 7.82 & 7.93 & 2.34 \\
\hline $1.75-2.00$ & 2.390 & 8.78 & 5.81 & 6.16 & 2.32 \\
\hline $2.00-2.25$ & 2.955 & 6.20 & 4.51 & 3.71 & 2.06 \\
\hline $2.25-2.50$ & 3.917 & 5.17 & 3.52 & 3.41 & 1.66 \\
\hline $2.50-2.75$ & 4.718 & 4.72 & 2.96 & 3.37 & 1.48 \\
\hline $2.75-3.00$ & 5.505 & 4.38 & 2.57 & 3.33 & 1.19 \\
\hline $3.00-3.25$ & 5.127 & 4.38 & 2.57 & 3.37 & 1.13 \\
\hline $3.25-3.50$ & 4.386 & 4.79 & 2.67 & 3.78 & 1.21 \\
\hline $3.50-3.75$ & 3.045 & 5.16 & 3.16 & 3.90 & 1.20 \\
\hline $3.75-4.00$ & 1.618 & 5.99 & 4.33 & 3.64 & 1.97 \\
\hline $4.00-4.25$ & 0.741 & 10.09 & 6.00 & 7.87 & 1.95 \\
\hline $4.25-4.50$ & 0.249 & 28.90 & 10.41 & 24.77 & 10.64 \\
\hline $4.50-4.75$ & 0.029 & 61.96 & 39.88 & 46.18 & 10.78 \\
\hline
\end{tabular}

Table 6.4: All errors. The normalization errors - luminosity (1\%) and tracking $(2.48 \%)$ are the same in every bin 


\begin{tabular}{|c|c|c|c|c|c|}
\hline $\begin{array}{c}\text { Momentum } \\
\text { Range }\end{array}$ & $\begin{array}{c}\text { Fitting } \\
(\%)\end{array}$ & $\begin{array}{c}\text { Uncor. MC } \\
\text { Efficiency }(\%)\end{array}$ & $\begin{array}{c}\text { PID } \\
(\%)\end{array}$ & $\begin{array}{c}\text { MC/Data } \\
\text { Shift }(\%)\end{array}$ & $\begin{array}{c}\text { Migration } \\
(\%)\end{array}$ \\
\hline $0.00-0.25$ & 15.21 & 14.02 & 1.66 & 1.45 & .18 \\
$0.25-0.50$ & 51.88 & 4.40 & 1.86 & 1.35 & .10 \\
$0.50-0.75$ & 19.60 & 0.00 & 1.91 & 1.25 & .14 \\
$0.75-1.00$ & 9.27 & 2.74 & 1.69 & 1.15 & .23 \\
$1.00-1.25$ & 4.27 & 3.41 & 1.61 & 1.05 & .40 \\
$1.25-1.50$ & 3.40 & 3.89 & 1.55 & .95 & .59 \\
$1.50-1.75$ & 6.69 & 3.69 & 1.62 & .85 & .67 \\
$1.75-2.00$ & 4.80 & 3.25 & 1.65 & .75 & .59 \\
$2.00-2.25$ & 1.67 & 2.58 & 1.67 & .65 & .59 \\
$2.25-2.50$ & 1.13 & 2.42 & 1.72 & .55 & .64 \\
$2.50-2.75$ & 1.58 & 2.04 & 1.77 & .50 & .67 \\
$2.75-3.00$ & 1.67 & 1.90 & 1.82 & .50 & .59 \\
$3.00-3.25$ & 1.86 & 1.73 & 1.85 & .50 & .64 \\
$3.25-3.50$ & 2.60 & 1.64 & 1.88 & .50 & .48 \\
$3.50-3.75$ & 2.67 & 1.71 & 1.94 & .71 & .27 \\
$3.75-4.00$ & 2.33 & 1.42 & 1.93 & 1.12 & .17 \\
$4.00-4.25$ & 6.67 & 3.20 & 2.01 & 1.54 & .12 \\
$4.25-4.50$ & 24.58 & 0.00 & 2.05 & 1.95 & .46 \\
$4.50-4.75$ & 43.81 & 14.19 & 2.13 & 2.37 & .79 \\
\hline
\end{tabular}

Table 6.5: Independent errors. The Dalitz structure error (.9\%) is the same in every bin. 


\section{CHAPTER 7}

\section{Comparisons with Previous Experiments and Models}

In this chapter we will compare our momentum spectrum to the prediction of the BaBar Monte Carlo, a previous measurement made by the CLEO experiment, and to the fragmentation models discussed in chapter 2 .

The BaBar experiment uses the JETSET/Pythia Monte Carlo, tuned to fit the BaBar data, to generate continuum events. Since our corrections are based on this Monte Carlo, we would like to see agreement between its prediction and our measured spectrum. However, if the two are not consistant, we have already checked the sensitivity of our measurement to shifts in the Monte Carlo spectrum in figure 6.12 .

Figure 7.1 shows our measurement in black and the BaBar Monte Carlo generator level spectrum in red. As the spectra are normalized to unit area, the normalization error is not included. The figure shows that the Monte Carlo does not match the narrow peak seen at $3 \mathrm{GeV} / \mathrm{c}$ in the data and also overpredicts the rate at high momentum. We will see that these features occur in several of the other hadronization models.

Figure 7.2 shows the previous best measurement of the $\Lambda_{c}$ spectrum from the CLEO experiment [24]. This measurement was done in 1991 and used only $101 p b^{-1}$ of data. The $\Lambda_{c} \rightarrow p K^{0}$ (Branching Fraction $2.3 \pm 0.6 \%$ ) and $\Lambda_{c} \rightarrow$ 


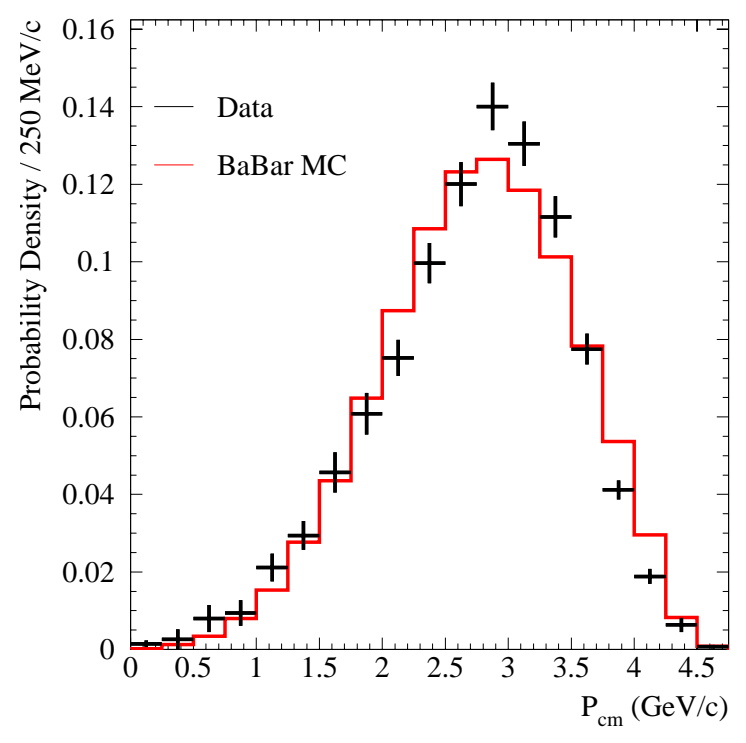

Figure 7.1: Comparison of Data with the BaBar Monte Carlo

$\Lambda \pi$ (Branching Fraction $0.9 \pm 0.28 \%$ ) modes are included with $\Lambda_{c} \rightarrow \pi K p$ (Branching Fraction $5.0 \pm 1.3 \%$ ). The CLEO measurement is consistant with ours, but, due to lower statistics, the errors are much larger.

Now we will compare the various fragmentation models discussed in Chapter 2 with our results. The model spectra are created at the generator level, without a detector simulation, and compared to our corrected data spectrum. We will judge the goodness of each model by its $\chi^{2} / D o F$, which, because of the correlated errors, must be calculated using the full error matrix

$$
\chi^{2}=(\mathbf{D}-r \mathbf{M})^{T} \frac{1}{\sigma^{2}}(\mathbf{D}-r \mathbf{M})
$$

where $\mathbf{D}$ is a column vector of the the number of corrected data entries in each bin, $\mathbf{M}$ is the number of model entries, $\mathrm{T}$ is the transposition operator, $\mathrm{r}$ is a scale factor such that the model and data have the same total rate integrated over momentum, and $\sigma^{2}$ is the error matrix. 


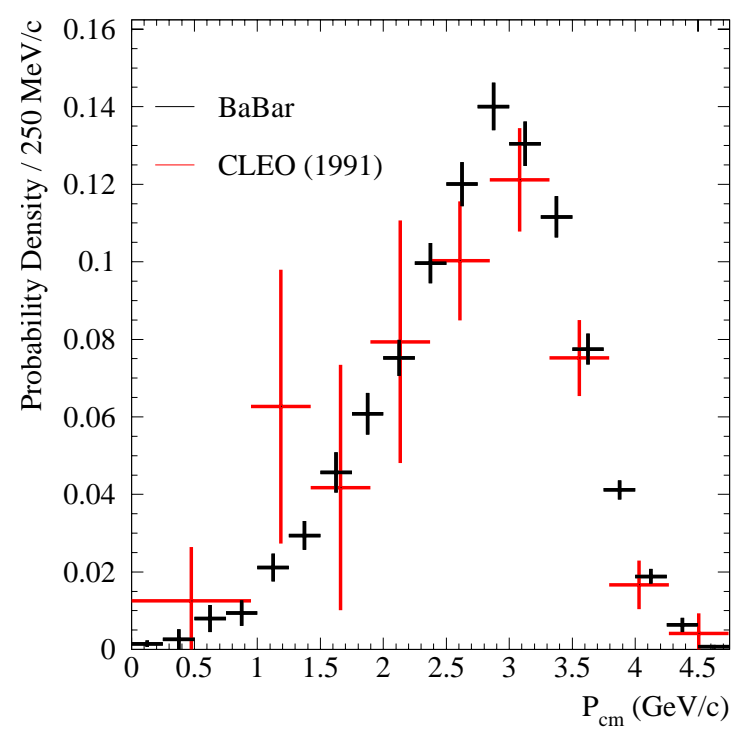

Figure 7.2: Comparison of our Data with the previous best measurement, by CLEO.

The off diagonal terms of the error matrix are shown in table 6.3, measured in percent of $\mathbf{M}$. The diagonal errors of the correlated error matrix are added in quadrature to the non-correlated errors to get the diagonal term of $\sigma^{2}$.

The calculation of the non-correleated errors, $\sigma_{i}$, is a bit tricky. The errors reported on an experimental measurement are the expected variance of the measurement given that the expected mean value is equal to the actual experimental result. When comparing with a model, however, the expected mean value of the measurement is equal to the prediction of the model, and the expected variance must be scaled accordingly. In a simple counting experiment with no systematics, the errors scale like $\sqrt{n}$. Our error calculation is made more complicated because of the background subtraction required to extract the $\Lambda_{c}$ signal.

Because we are only testing the models' ability to reproduce the $\Lambda_{c}$ momentum 
spectrum, we will assume that they generate the same backgrounds as exist in the data. The only change is the size of the $\Lambda_{c}$ mass peak in each momentum bin. In order to estimate the statistical error for various signal sizes we first simulate the expected invariant mass plots by adding or subtracting a randomly generated Gaussian of appropriate width and center to the data. The signal size is varied by geometrical factors of 1.2 from $\left(\frac{1}{1.2}\right)^{10}$ to $1.2^{10}$. The resulting histograms are then fit using the same procedure as for the data and the systematic errors are put into a lookup table. The results for four different momentum regions are shown in figure 7.3. It can be seen that the statistical error is much less sensitive to the signal size for slow $\Lambda_{c}$. This is because at low momenta, the statistical error on the invariant mass fits is dominated by sideband subtraction (figure 6.3). In the $4.25 \leq P_{c m} \leq 4.50$ bin, where the background is very low, we see that a four fold increase in signal size increases the statistical error by a factor of 1.9, which is close to the factor of 2.0 expected from an experiment with only counting errors.

The point to point systematic errors shown in table 6.5 also need to be scaled up to what they would have been if the number of observed events were equal to the model prediction.

$$
\sigma_{\text {model systematic }}=\sigma_{\text {data systematic }} \frac{r M}{D}
$$

We also have an additional error due to the statistics of the generated spectrum

$$
\sigma_{\text {generator statistics }}=r \sqrt{M}
$$

although, due to the speed of the generator, this can be made much smaller than the other errors. Summing the three pieces, we get a total non-correlated 


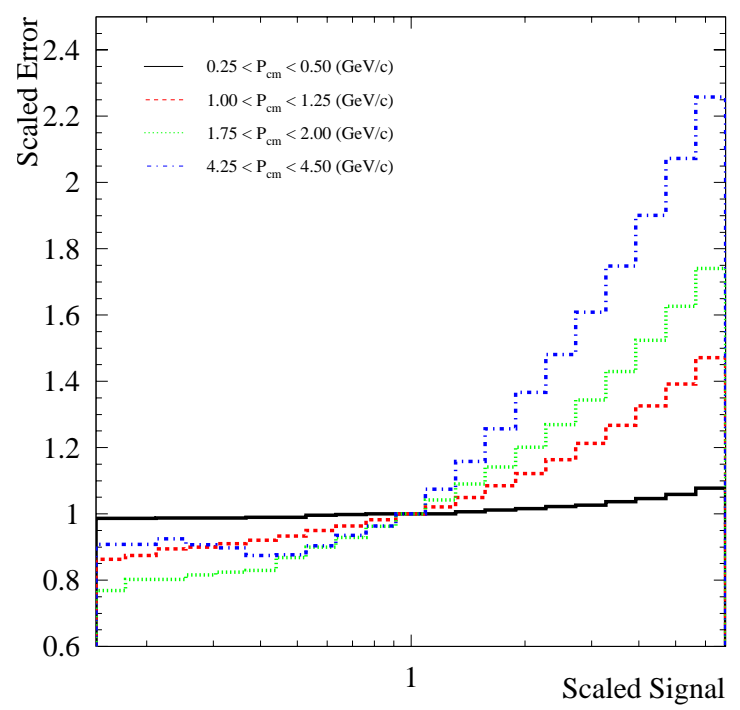

Figure 7.3: Expected scaling of statistical errors for four momentum regions

error of

$$
\sigma^{2}=(\text { Statistical Error Table })^{2}+\sigma_{\text {data systematic }}^{2} \frac{r^{2} M^{2}}{D^{2}}+r^{2} M
$$

In practice the only case where there is a noticeable difference between the reported data error and $\sigma$ is when the model overpredicts the data by a large fraction above $4 \mathrm{GeV} / \mathrm{c}$. At low momenta the error is dominated by the constant sideband subtraction error.

The results of the model comparisons are shown in figure 7.4. These models are described in detail in section 2.2. The first seven models are all created using the JETSET Monte Carlo program with a modified fragmentation function. The form of the functions are shown in table 7.1. The remaining models, HERWIG and UCLA are generated by separate programs. Table 7 shows the $\chi^{2} / D o F$ for each model, along with the values of the parameters giving he best fit, and the average momentum. 


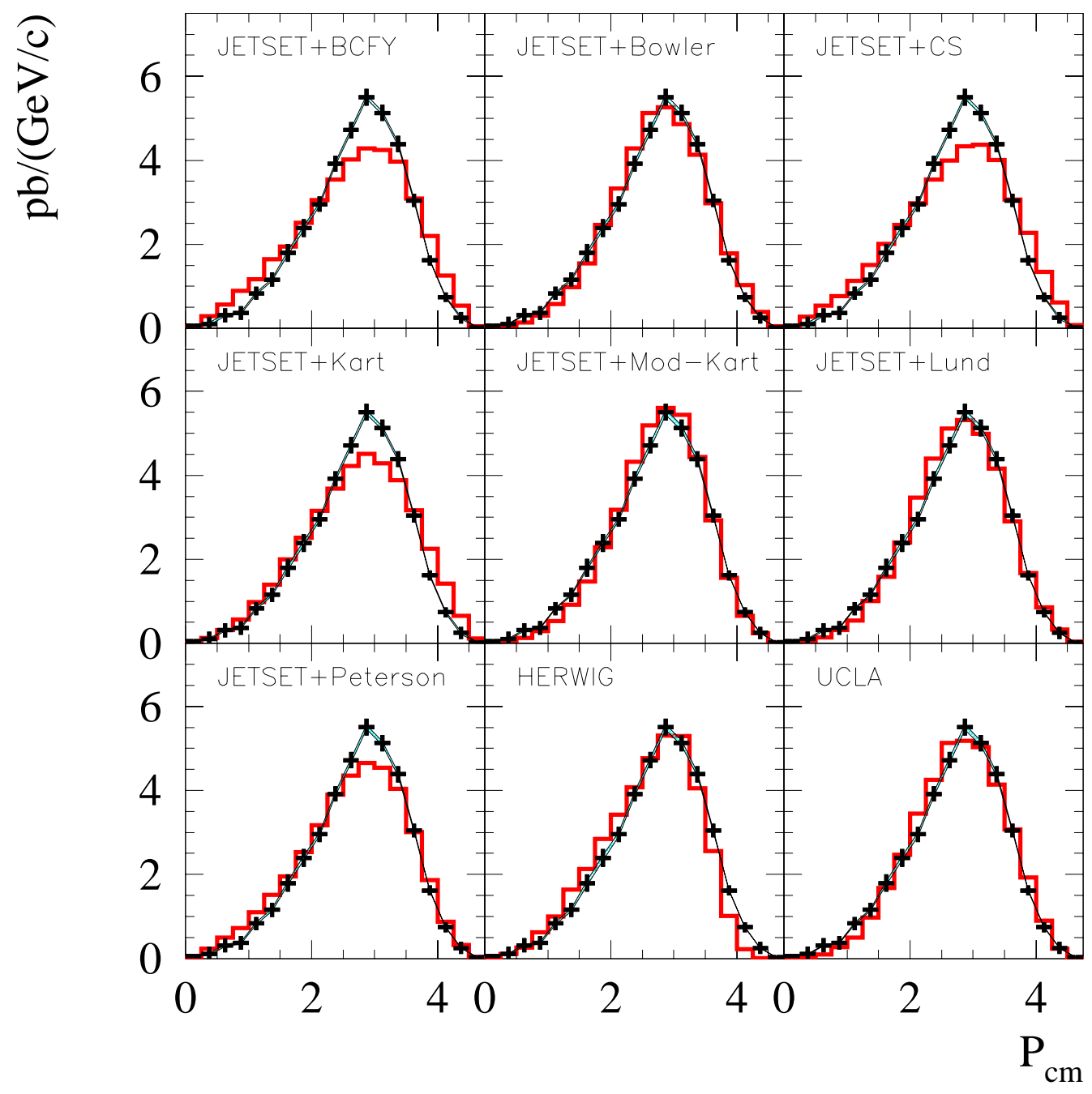

Figure 7.4: Comparison of the hadronization models, in red, with the data, in black. Correleated errors are shown as a blue band. Uncorrelated errors are shown as error bars. 
Four of the five one-parameter models (BCFY, Collins and Spiller, Kartivelishvili, and Peterson) fail to reproduce the narrow peak of the $\Lambda_{c}$ spectrum seen in the data. The exception, the modified Kartivelishvili function, is the only one that takes into consideration the extra degree of freedom coming from the additional spectator quark in baryon formation. It would be interesting to see how baryon versions of the other three models would compare with data, but unfortunately, they are not currently available.

The two parameter models - Lund, Bowler and UCLA, do a better job of fitting the central region of the spectrum. It should be noted that the two parameters in these models are highly correlated, so that a wide range of correlated parameter values give similar results. For example changing the Lund model parameters from $\mathrm{a}=1.00$ and $\mathrm{b}=.62$ to $\mathrm{a}=.69$ and $\mathrm{b}=.55$ only raises the $\chi^{2}$ from 28 to 35 .

It is not clear that $\chi^{2} / D o F$ is the best way of judging these models. The HERWIG model is by far the worst in terms of $\chi^{2} / D o F$, because its predictions at high momentum, where the absolute errors are small, differ by several sigma. The overall shape of the HERWIG spectrum, however, might be considered to be better than the models that fail to reproduce the narrowness of the peak.

Figure 7.5 shows a potential source of error in the models' momentum predictions. The BaBar Monte Carlo generated spectrum of $\Lambda_{c}$ from string decay differs noticeably from that of $\Lambda_{c}$ coming from decays of $\Sigma_{c}$. This is because the heavier $\Sigma_{c}$ baryon has a lower $p_{\max }$ than the $\Lambda_{c}$ and because additional momentum is carried off by the daughter pion. The current monte carlo generators do not contain all of the heavy charmed baryons, and their mutiplicities are not well known. It would be an interesting future exercise to retune the models so that they get the relative rates of different charmed baryons to match with data and 


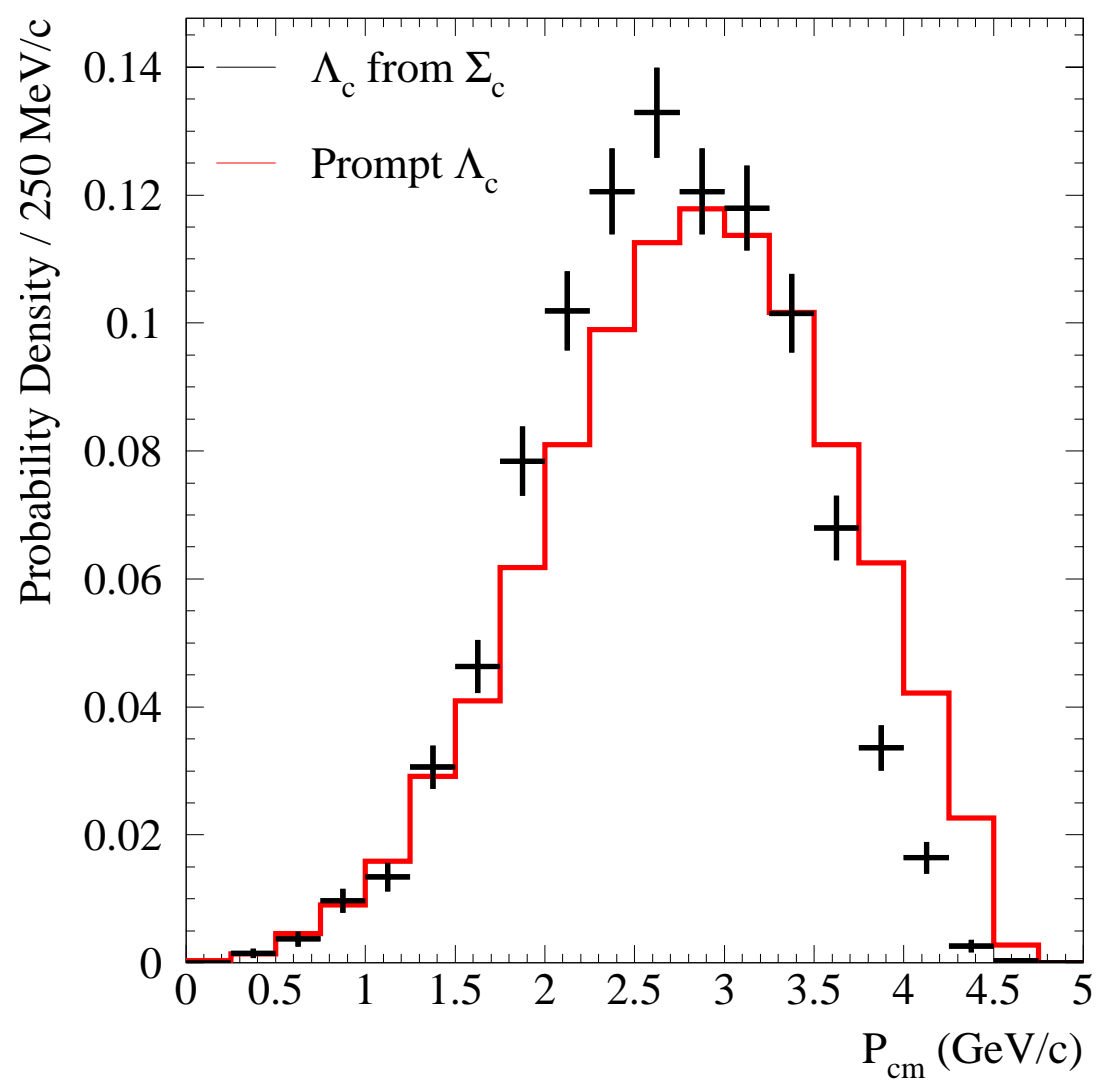

Figure 7.5: JETSET prompt $\Lambda_{c}$ spectrum vs. the $\Lambda_{c}$ from $\Sigma_{c}$ spectrum

see how the inclusive $\Lambda_{c}$ momentum spectrum predictions change. 


\begin{tabular}{|c|c|c|}
\hline Model & $f(z, \beta)$ & Reference \\
\hline BCFY & $\frac{z(1-z)^{2}}{[1-(1-r) z]^{6}}\left[3+\sum_{i=1}^{4}(-z)^{i} f_{i}(r)\right]$ & {$[12]$} \\
\hline Bowler & $\frac{1}{z^{\left(1+b m_{\perp}^{2}\right)}}(1-z)^{a} \exp \left(-b m_{\perp}^{2} / z\right)$ & {$[10]$} \\
\hline CS & $\left(\frac{1-z}{z}+\frac{(2-z) \epsilon_{b}}{1-z}\right)\left(1+z^{2}\right)\left(1-\frac{1}{z}-\frac{\epsilon_{b}}{1-z}\right)^{-2}$ & {$[11]$} \\
\hline Kartvelishvili & $z^{\alpha_{b}}(1-z)$ & {$[9]$} \\
\hline Modified & & \\
\hline Kartvelishvili & $z^{\alpha_{b}}(1-z)^{3}$ & chap. 2 \\
\hline Lund & $\frac{1}{z}(1-z)^{a} \exp \left(-b m_{\perp}^{2} / z\right)$ & {$[6]$} \\
\hline Peterson & $\frac{1}{z}\left(1-\frac{1}{z}-\frac{\epsilon_{b}}{1-z}\right)^{-2}$ & {$[7]$} \\
\hline
\end{tabular}

Table 7.1: $c$-quark fragmentation models used in comparison with the data. For the BCFY model, $f_{1}(r)=3(3-4 r), f_{2}(r)=12-23 r+26 r^{2}$, $f_{3}(r)=(1-r)\left(9-11 r+12 r^{2}\right)$, and $f_{4}(r)=3(1-r)^{2}\left(1-r+r^{2}\right)$. 


\begin{tabular}{|l|c|c|c|}
\hline Model & $\chi^{2} /$ dof & Parameters & $\langle p\rangle$ \\
\hline JETSET + BCFY & $131 / 18$ & $r=0.35$ & 2.66 \\
JETSET + Bowler & $35 / 17$ & $a=.63, b=.73$ & 2.76 \\
JETSET + CS & $124 / 18$ & $\epsilon_{b}=0.12$ & 2.69 \\
JETSET + Kartvelishvili et al. & $106 / 18$ & $\alpha_{b}=3.1$ & 2.73 \\
JETSET + Modified Kart. & $29 / 18$ & $\alpha_{b}=7.65$ & 2.75 \\
JETSET + Lund & $29 / 17$ & $a=1.00, b=0.62$ & 2.76 \\
JETSET + Peterson et al. & $50 / 18$ & $\epsilon_{b}=0.077$ & 2.65 \\
HERWIG & $338 / 19$ & - & 2.59 \\
UCLA & $43 / 17$ & $a=2.1, b=.53$ & 2.77 \\
\hline
\end{tabular}

Table 7.2: The minimum $\chi^{2}$, number of degrees of freedom, corresponding parameter values, and the mean value of the corresponding $\Lambda_{c}$ momentum distribution are listed. 


\section{CHAPTER 8}

\section{$\Lambda_{c}^{+} \mid \bar{\Lambda}_{c}^{-}$Events}

In this chapter we will finally look for popcorn mesons in events with both a $\Lambda_{c}^{+}$ and a $\Lambda_{c}^{-}$. If we require each $\Lambda_{c}$ to have a momentum of greater than $2.3 \mathrm{GeV} / \mathrm{c}$ in the center of mass frame, we will see that the cosine of the angle between the momentum vectors is almost always more negative than -.9. This is what we expect to see in $c \bar{c}$ events where the $\Lambda_{c}$ are forming at the ends of the string. The Belle experiment has recently observed larger than expected numbers of events with more than one charmonium meson [26], implying that large amounts of charm can be produced from gluon fusion. However, in order to make a $\Lambda_{c}^{+}$and a $\Lambda_{c}^{-}$moving back to back with momenta of $2.3 \mathrm{GeV} / \mathrm{c}$, you would need a 6.5 $\mathrm{GeV}$ gluon, which we would not expect to see at BaBar energies.

So we have a set of events with a baryon-antibaryon pair separated by a large rapidity gap. First we will count the number of mesons we see in these events. Then we will try to put a limit on how many of the mesons could be coming from decays of excited baryons. Any remaining mesons will be popcorn.

\subsection{Selection of $\Lambda_{c}^{+} \mid \bar{\Lambda}_{c}^{-}$Events}

Because the $\Lambda_{c} \rightarrow \pi^{+} k^{-} p$ branching fraction is so small $(5.0 \pm 1.3 \%)$, and the rate of detected $\Lambda_{c}^{+} \mid \bar{\Lambda}_{c}^{-}$events is proportional to this branching fraction squared, we will try adding more $\Lambda_{c}$ decay modes to increase our sample size. 
- $\Lambda_{c} \rightarrow K^{0} p \quad \mathrm{BF}=2.3 \pm 0.6 \%$

- $\Lambda_{c} \rightarrow \Lambda \pi^{+} \quad \mathrm{BF}=0.9 \pm 0.28 \%$

- $\Lambda_{c} \rightarrow K^{0} p \pi^{+} \pi-\quad \mathrm{BF}=2.6 \pm 0.7 \%$

- $\Lambda_{c} \rightarrow \Lambda \pi^{+} \pi^{+} \pi^{-} \quad \mathrm{BF}=3.3 \pm 1.0 \%$

In addition to the particle identification cuts for charged and neutral particles described in chapter 4 , we apply the following cuts.

- Charged particle tracks have at least 5 hits in the SVT.

- Charged particle tracks have at least 15 hits in the DCH. This cut is lowered from the requirement of 20 hits in spectrum measurement in order to increase the signal size.

- The probability that the daughters of the $\Lambda_{c}$ come from a common vertex must be greater than $.001 \%$ for all modes except $\Lambda_{c} \rightarrow K^{0} p$. The $\Lambda_{c}^{+} \mid \bar{\Lambda}_{c}^{-}$ analysis is done with a newer version of the BaBar software, which correctly accounts for particle energy lost in interactions with the detector material. The vertexing software was not reliable in the previous version, where this cut had to be replaced by a cut on the distance of closest approach to the global event vertex.

- The center of mass momentum of the $\Lambda_{c}$ must be greater than $2.3 \mathrm{GeV} / \mathrm{c}$, which is the kinematic limit for $\mathrm{B}$ decays to $\Lambda_{c}$. This cut also reduces the amount of energy available for the creation of extra particles, which is good because we are interested in finding events without compensating baryons in each jet. An interesting future study would be to relax the $2.3 \mathrm{GeV} / \mathrm{c}$ momentum cut and see how baryon compensation varies with the available energy. 
The invariant mass plot for each of the five modes in shown in figure 8.1. The fits, shown in red, are just a single Gaussian with a linear background. The width of the peak increases for decays to neutral particles. It decreases for the five body modes, because each daughter is now slower, and reconstructed with a better resolution. The plots showing the decays to a $\Lambda$ have two reflections that we will have to take into consideration when doing the sideband subtraction. One is the decay $\Lambda_{c}^{+} \rightarrow \Sigma \pi^{+}$, where the $\Sigma$ decays to $\Lambda \gamma$. The edges of this reflection are marked with a blue box below the $\Lambda_{c}$ mass. The other is $\Xi_{c}^{0} \rightarrow \Xi^{-} \pi^{+}, \Xi^{-} \rightarrow \Lambda \pi^{-}$ where the $\Lambda$ is combined with the $\pi^{+}$. This reflection is above the $\Lambda_{c}$ mass, but the rate is too small to be seen in the plots. The sidebands in the other modes are very flat, which means that we can estimate the density of background under the peak by simply averaging the height of the sidebands.

At this point we attempted to optimize the width of our signal region cut for each of these modes separately in such a way as to minimize the average statistical error on the popcorn candidate helicity angle plot (figure 8.20). It was found that the inclusion of all five modes only reduced the statistical error to $96.3 \%$ of what it was using the $\pi^{+} k^{-} p$ and $K^{0} p$ modes only. This does not include any additional errors that would come from the subtraction of the reflections, so we decided to do the analysis using only the $\pi^{+} k^{-} p$ and $K^{0} p$ modes.

Using only these two modes, we search for events containing both a $\Lambda_{c}^{+}$and a $\bar{\Lambda}_{c}^{-}$. The invariant mass of each $\Lambda_{c}^{+} \bar{\Lambda}_{c}^{-}$pair having center of mass momentum vectors with a negative dot product is plotted in fig 8.2. This cut eliminates events where an anti-proton locally compensating the $\Lambda_{c}^{+}$baryon number is combined with two random tracks to make a fake $\Lambda_{c}^{-}$in the same hemisphere. A close-up of the signal region is shown in fig 8.3. The central circle of radius $12 \mathrm{MeV}$ is the signal region. The circles to each side are used to estimate the one fake rate and 

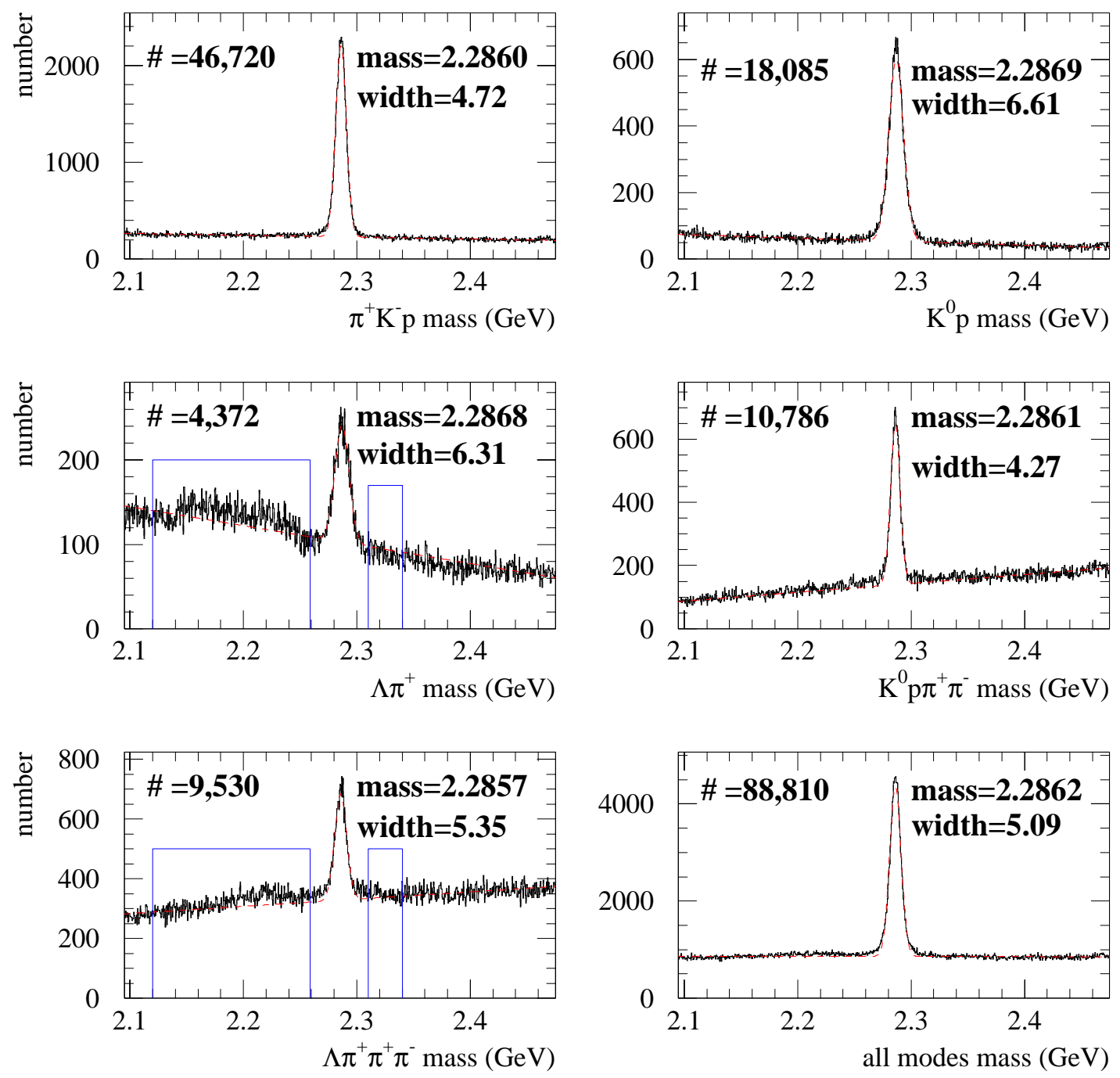

Figure 8.1: Invariant mass distributions in the region of the $\Lambda_{c}$ mass for five different combinations of particles known to be $\Lambda_{c}$ modes. The red lines represent a simple Gaussian fit, and the blue lines show the regions of the reflections described in the text. 


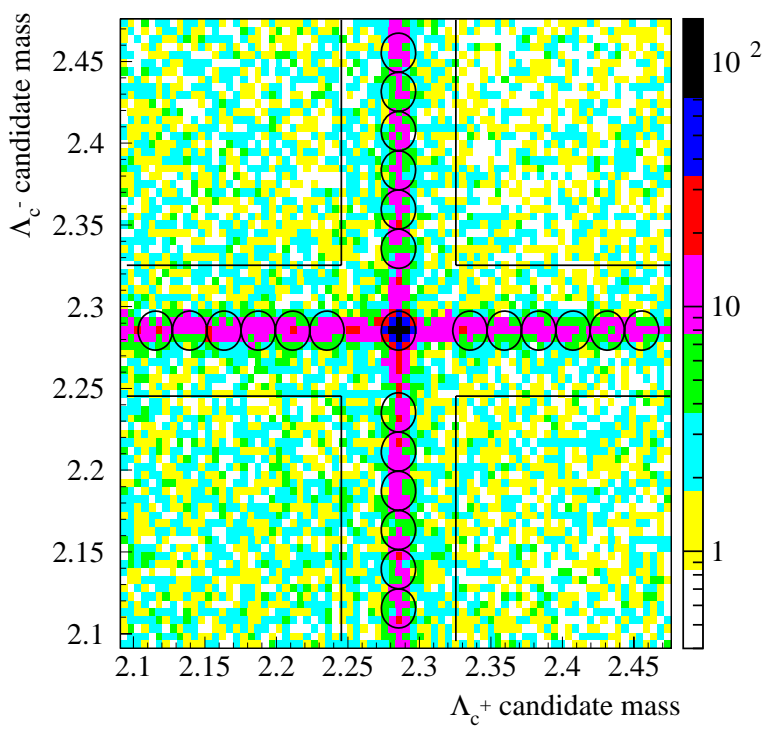

Figure 8.2: Invariant masses of $\Lambda_{c}^{+} \bar{\Lambda}_{c}^{-}$candidate pairs for which the dot product of the center of mass momenta is negative.

the four off-axis square regions are used to estimate the two fake rate. The change in signal and background versus the radius of the cut is shown in fig 8.4. The blue line in this figure shows how the increased statistics will affect the average error over the ten bins in figure 8.20. The cut of $12 \mathrm{MeV}$ on the radius gets us close to the minumum statistical error value, without unnecessarily increasing the amount of background subtraction. This yields 649 signal events after sideband subtraction with one and two fake rates of 245 and 25. The signal to background ratio is 2.4 .

The particles in each event which do not come from a $\Lambda_{c}$ will be potential popcorn candidates. The following cuts are applied to these tracks to make sure they are real and to distinguish them from machine backgrounds. The tracking cuts applied to these tracks are 


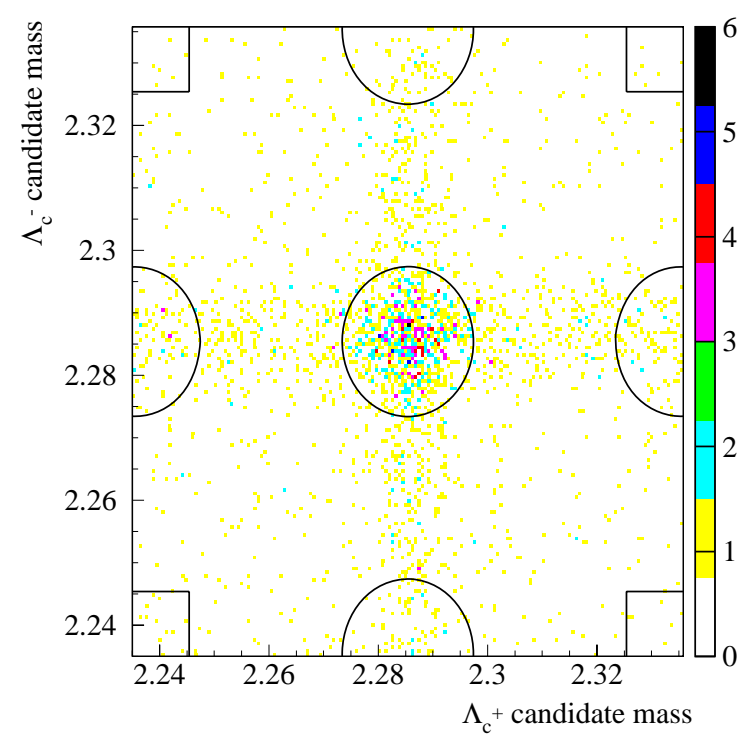

Figure 8.3: Closeup of the invariant masses of $\Lambda_{c}^{+} \bar{\Lambda}_{c}^{-}$candidate pairs for which the dot product of the center of mass momenta is negative.

- at least 5 hits in the SVT

- at least 10 hits in the DCH

- the distance of closest approach of the track to the beam in the transverse plane is less than $5 \mathrm{~mm}$.

The SVT and distance of closest approach cuts are necessary to reduce the number of non physics tracks due to beam interactions and photon conversions. The requirement of $10 \mathrm{DCH}$ hits per track is needed to prevent large numbers of single real tracks from being reconstructed as two separate tracks. This currently happens at a rate of once every 246 tracks in the Monte Carlo.

There is a possiblity that there will be $K_{s}$ popcorn mesons. Since these particles can have flight lengths of several centimenters, their daughter pions will 


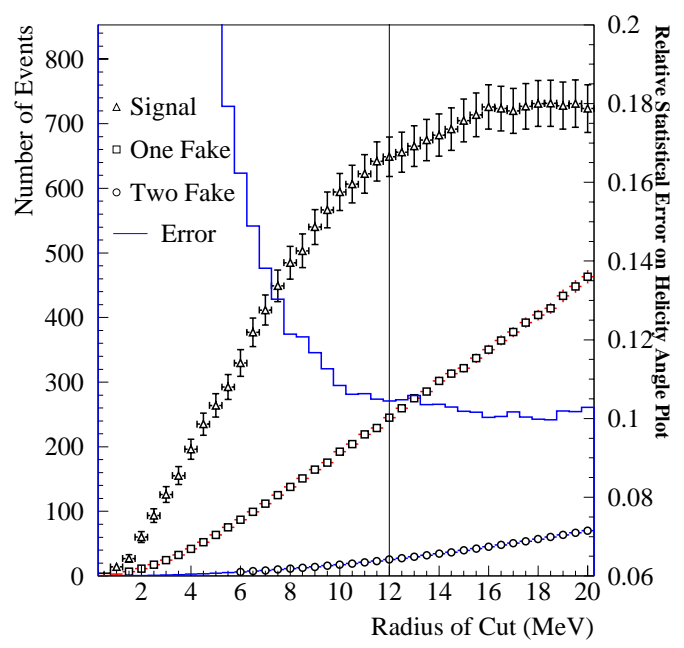

Figure 8.4: Sideband-subtracted signal and background rates vs. the radius of the cut used in figure 8.3. The blue line shows the corresponding average statistical error on the helicty angle plot (figure 8.20).

not necessarily pass the tracking cuts mentioned above. We will specifically look for these cases in section 8.5.

Some interesting event variables are shown in figure 8.5. The background subtraction for these and all following plots are done by weighting the sideband events by the ratio of the signal region area to the sideband area. The center of mass momentum distribution is cut off at $2.3 \mathrm{GeV} / \mathrm{c}$ to get rid of $\mathrm{B}$ decays and drops to zero around the kinematic limit of $4.74 \mathrm{GeV} / \mathrm{c}$ as expected. The second plot shows the cosine of the angle between the $\Lambda_{c}^{+}$and $\bar{\Lambda}_{c}^{-}$. The signal $\Lambda_{c}$ are anticolinear more often than the background, so we could get a cleaner signal by placing a hard cut on the angle. We have decided not to do this as it might bias us against events with large amounts of popcorn, which we would expect to have greater transverse momentum. 

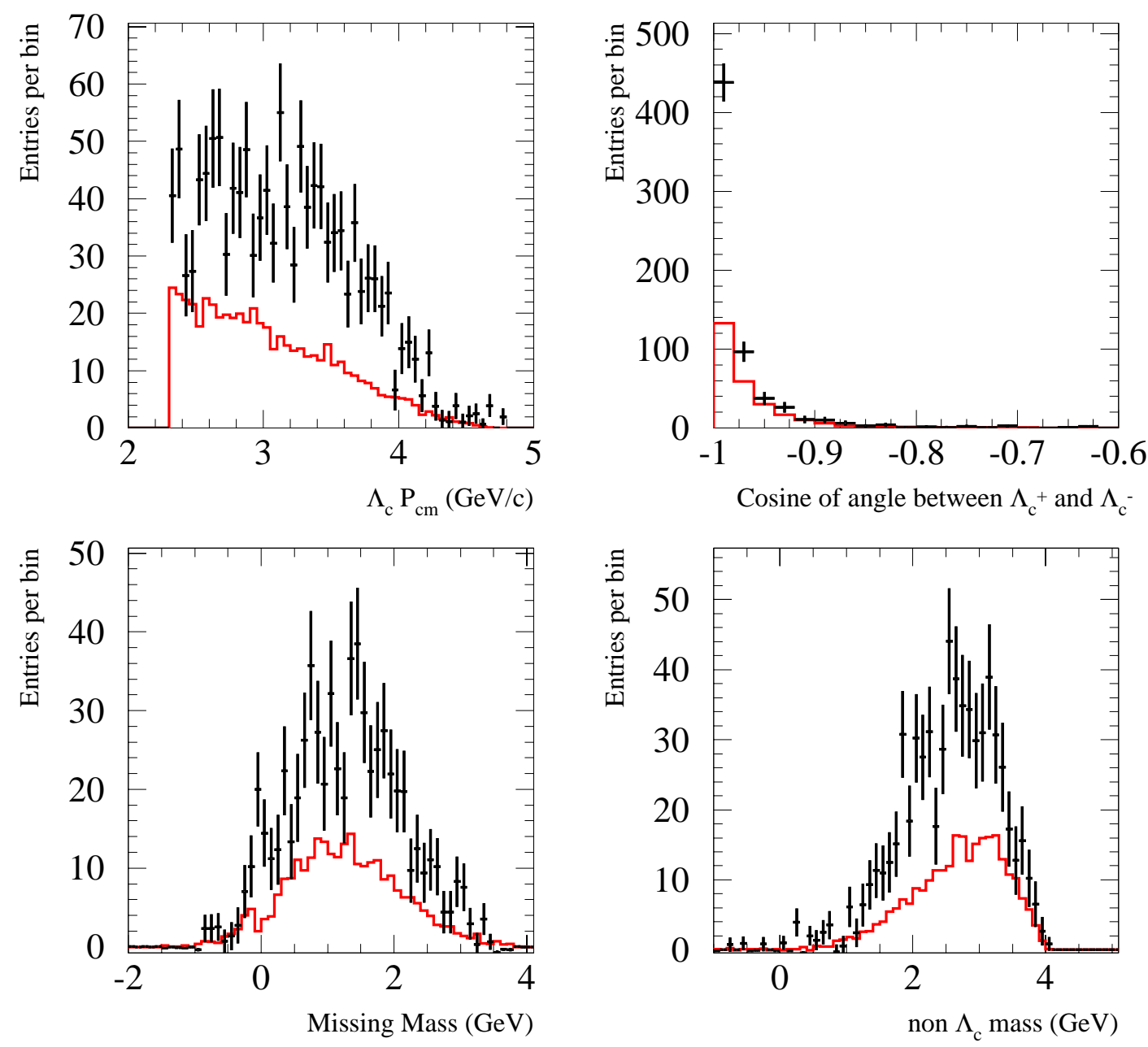

Figure 8.5: Center of mass momentum, Angle between the $\Lambda_{c}^{+}$and $\bar{\Lambda}_{c}^{-}$, Missing mass and non $\Lambda_{c}$ mass. The sideband-subtracted signal is in black. The sideband is in red. 
The third plot, the missing mass, shows the mass of the 4-vector momentum sum of all charged tracks minus the beam momentum 4-vector. This is the invariant mass of all neutral particles and the charged particles that do not pass the tracking cuts. Imaginary masses are plotted on the negative real axis. There appears to be a small peak at zero, representing events that have been fully reconstructed. The resolution of the missing mass measurement might be as high as a few hundred $\mathrm{MeV}$, but events with missing masses less than $-0.5 \mathrm{GeV}$ must contain spurious tracks or misidentified or poorly reconstructed tracks and will be cut out of the final event sample. The fact that the missing mass peaks below $2 \mathrm{GeV}$ shows that we do not have a large percentage of events with two neutrons. This, combined with the small number of identified protons that will be shown in figure 8.6, confirms the CLEO observation that the $\Lambda_{c}$ do not have to be compensated by a same jet anti-baryon, wheras both the JETSET and UCLA Monte Carlos are forced to do this by their construction.

The final plot in figure 8.5, the non- $\Lambda_{c}$ mass, shows the mass of the beam momentum 4-vector minus those of the $\Lambda_{c}^{+}$and $\bar{\Lambda}_{c}^{-}$. The dearth of entries near zero shows that we do not often find events containing only a $\Lambda_{c}^{+}$and a $\bar{\Lambda}_{c}^{-}$and nothing else. Again, events corresponding to a value of less than $-0.5 \mathrm{GeV}$ must have a reconstruction problem and so will be thrown out.

Figure 8.6 shows the number of charged tracks per event after sideband subtraction not coming from a $\Lambda_{c}$ decay per event. Two interesting features can be seen in this plot. The first is that there is a very small number of identified protons (kaons). Of 1,774 charged tracks, 30 (46) are identified as protons (kaons). If all of these tracks were really misidentified pions, this would correspond to a missID rate of $1.7 \%(2.6 \%)$. Using the PID corrections from the control samples used in the inclusive measurement, we would expect a missID rate of $.1 \pm .08 \%$ 

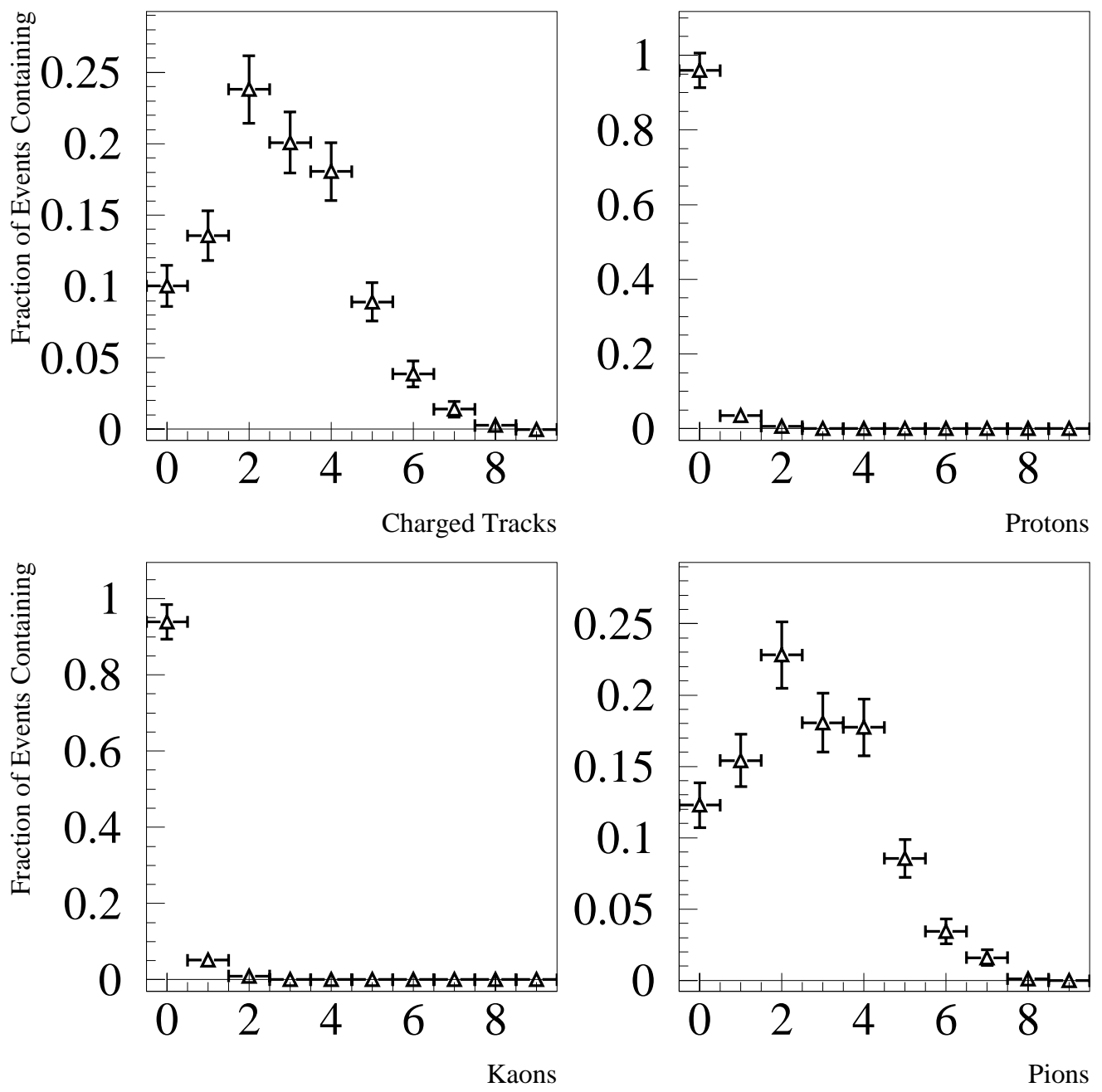

Figure 8.6: Fractions of events containing various numbers of additional charged tracks, and identified protons, kaons and pions in the $\Lambda_{c}^{+} \mid \bar{\Lambda}_{c}^{-}$events. 
$(.42 \pm .17 \%)$. These control samples required $20 \mathrm{DCH}$ hits instead of 10 , so they are probably not very reliable. We will see in the next section that a large fraction of the tracks identified as protons must be misidentified pions. As mentioned earlier, the fact that there are very few protons is a very interesting result, because it shows that baryon number does not have to be conserved in each jet separately.

The other interesting feature is the large number of events with four or more additional pions. These pions can come from one of two sources. Either they are coming from decays of heavier charmed baryons to $\Lambda_{c}^{+}+\mathrm{n} \pi$, or they are popcorn mesons. In section 8.3, we will study the expected number of pions from heavy charmed baryon decays.

\subsection{Four Baryon Events}

In this section we will try to estimate how many of our selected events contain two additional baryons. Since we are interested in measuring the amount of popcorn between a single baryon-antibaryon pair, we would like to subtract these events out of our sample. Figure 8.7 a) shows the missing mass in events with one and two identified protons. Of the 22.4 events with one identified proton, 7.2 of them have a missing mass of less than $750 \mathrm{MeV}$. Since another baryon with a mass of at least $938 \mathrm{MeV}$ would have to be present in these events, the proton identification must be wrong. When we assign the proton mass to a real pion or kaon, this lowers the amount of missing mass in the event. This explains why we see events with negative missing mass. The events with two identified protons have a missing mass close enough to zero that correct particle identification cannot be ruled out. If both of the protons in these events were misidentified pions or kaons, we might end up with a very negative missing mass. 
Now, of the events in figure 8.7 a) with one identified proton and a missing mass of greater than $750 \mathrm{MeV}, 11.3$ of the protons are positively charged, and 3.9 are antiprotons. We expect a charge asymmetry in protons due to pion interactions with the positively charged protons in the detector material. So it looks like the events with one identified proton consist of about four physics protons and four physics antiprotons, with the rest coming from detector interactions.

If we assume that there are 5 double neutron events, 10 proton-neutron events, and 5 double proton events and a tracking efficiency of $75 \%$ then we would expect to see about 9.4 events with one proton and 2.8 events with two protons. This is consistant with what we see, 7.8 events with one proton and 3.1 events with two protons. In this case there would be 20 real four baryon events, compared to the 10.9 that we observe. This would leave 9.1 unobserved four baryon events that would need to be subtracted from our data sample. However, since a significant number of the identified protons in the one-proton events with a missing mass of greater than $750 \mathrm{MeV}$ are probably also misidentified pions, 9.1 is an overestimate. For the purposes of background subtraction, we assume there are $5 \pm$ 5 unobserved four baryon events in our sample, where the first 5 is an educated guess and the second 5 is a suitably large, arbitrary uncertainty.

Figure $8.7 \mathrm{~b}$ ) shows the number of identified pions in one-proton events with more than $750 \mathrm{MeV}$ of missing mass and two-proton events. We only see $.65 \pm .21$ extra pions in each of these events, compared to $2.65 \pm .08$ pions in the events with no observed baryons. We will make a small correction to our number of pions per two baryon event, in order to compensate for the events with unobserved extra baryons in section 8.6.

It is reasonable to expect fewer pions in the four baryon events, since there is less energy available for pion production. This effect is countered by the fact 

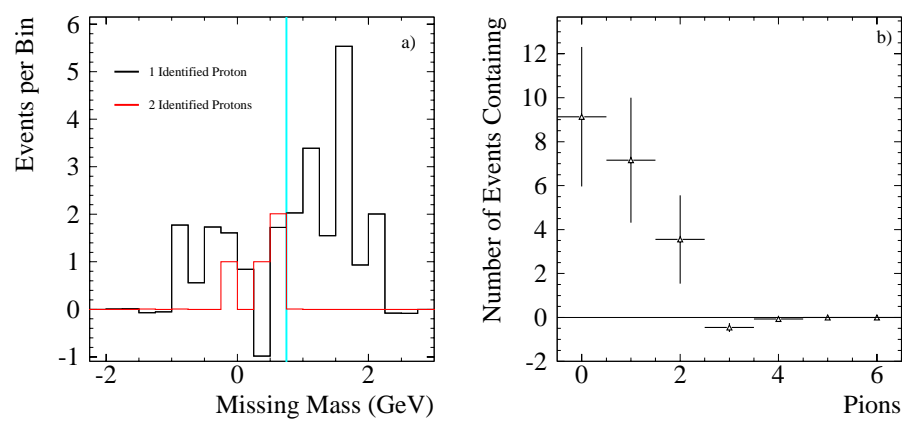

Figure 8.7: a) Missing mass in events with one and two identified protons. b) Number of pions in events where the existence of two baryons cannot be ruled out

that the popcorn mechanism is no longer required. In higher energy collisions, we would probably expect the number of pions to increase in four baryon events for this reason.

\subsection{Decays of Heavier Charmed Baryons}

In order to count the number of popcorn mesons in each $\Lambda_{c}^{+} \mid \bar{\Lambda}_{c}^{-}$event, we will first have to identify particles that could have come from the decay of a heavier charmed baryon. Fig 8.8 gives a summary of all previously seen charmed baryon decays to $\Lambda_{c}$. There are two $\Sigma_{c}$ states which decay to $\Lambda_{c}+\pi$ and four higher mass $\Lambda_{c}$ states that decay to $\Lambda_{c}+2 \pi$. Various $\Xi_{c}$ and $\Omega_{c}$ states have been seen, but these have always been seen to decay to strange baryons and not $\Lambda_{c}$. This is to be expected because of the much larger available phase space for decays to the lighter strange baryons and also because of Cabbibo suppression. The only particle ever seen to decay to a $\Lambda_{c}+\mathrm{K}$ is the doubly charmed $\Xi_{c c}^{+}(3519)$, but we 


\section{Mass (GeV)}

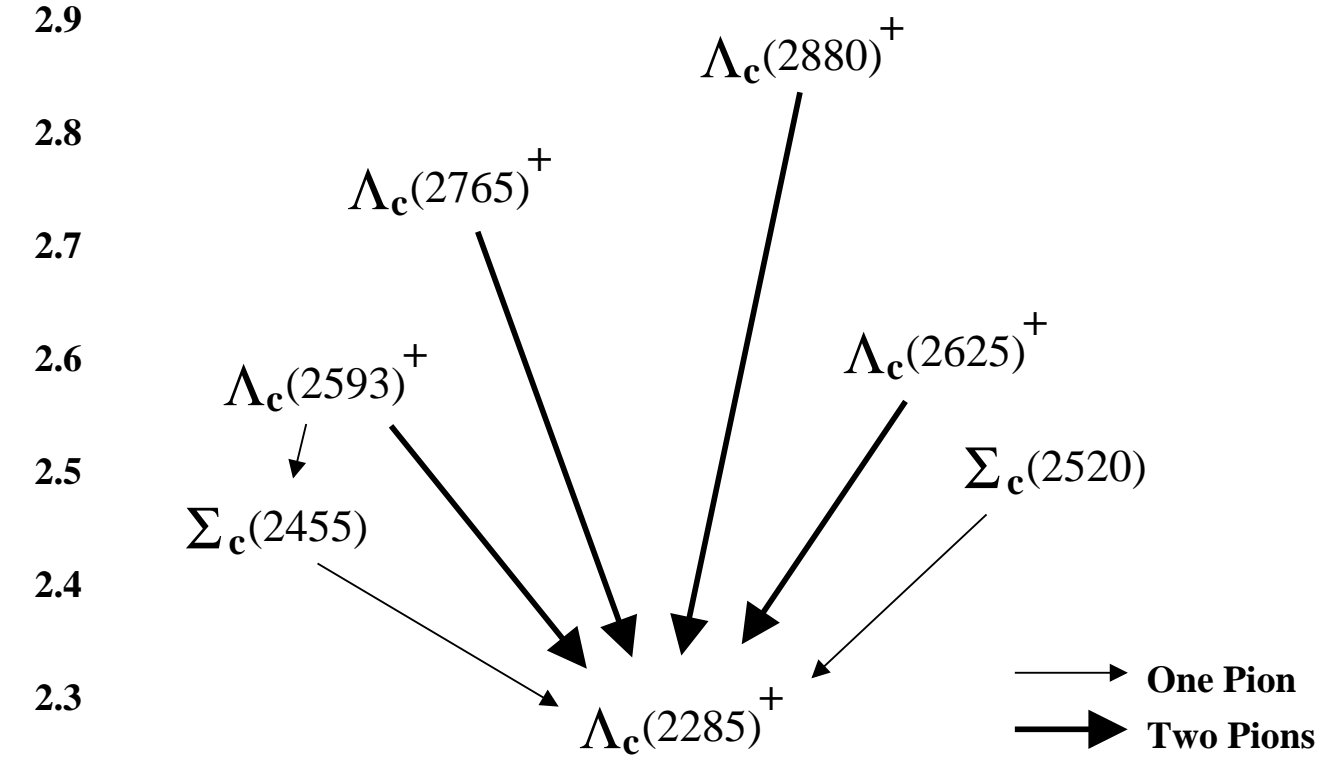

2.2

Figure 8.8: Previously observed decays to $\Lambda_{c}$.

do not expect this particle to be created at BaBar.

Figure 8.9 shows the invariant mass plots for $\Lambda_{c}+\pi$ and $\Lambda_{c}+2 \pi$. The regions between the green bars show the location of six charmed baryons from figure 8.8. We look for higher mass resonances by extending the invariant mass range in figure 8.10 , but do not find anything. The red plots in figure 8.9 are from the $\Lambda_{c}^{+} \mid \bar{\Lambda}_{c}^{-}$signal region with the sideband subtraction. In order to get a better estimate of these rates we would like to use the decays to the higher statistic sideband single $\Lambda_{c}$ sample (figure 8.2), but, because the efficiency for detecting pions from these decays depends on the lab momentum and angular distributions of the detected $\Lambda_{c}$, we will need to make sure that these are similar in the single and double $\Lambda_{c}$ events.

This turns out not to be the case, as can be seen in Fig 8.11. Backward 

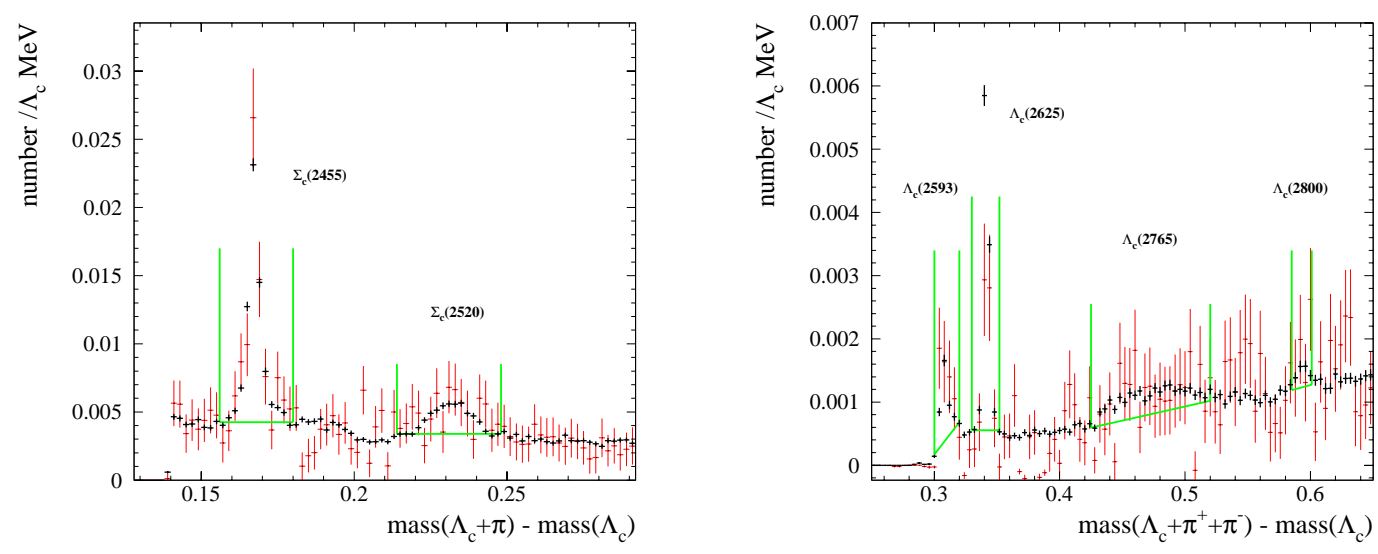

Figure 8.9: Comparison of the decays to $\Lambda_{c}$ in sideband subtracted $\Lambda_{c}^{+} \mid \bar{\Lambda}_{c}^{-}$events (red), to the higher statistic $\Lambda_{c}$ sample in the sidebands (black). The green lines roughly outline the signal regions.

moving $\Lambda_{c}$ are rarely seen in the $\Lambda_{c}^{+} \mid \bar{\Lambda}_{c}^{-}$events, because in this case the other $\Lambda_{c}$ would have been boosted out of the front of the detector. In order to correct for this, the single $\Lambda_{c}$ are weighted such that their distribution matches that of the $\Lambda_{c}^{+} \mid \bar{\Lambda}_{c}^{-}$signal.

Now we can use the single $\Lambda_{c}$ events to estimate the number of decay pions associated with each $\Lambda_{c}$. Figure 8.12 shows a closeup of the invariant mass regions near the four excited $\Lambda_{c}$ particles. Because of its proximity to the $\Sigma_{c}+\pi$ threshold, the $\Lambda_{c}(2593)$ has an unusual lineshape [28]. We will estimate the number of $\Lambda_{c}(2593)$ by simply counting the number of entries above the red line and assigning a $25 \%$ error. The $\Lambda_{c}(2593)$ is fit with a sum of two Gaussians, and the other two particles are fit with a single Gaussian. In order to be conservative on the estimated rates of the $\Lambda_{c}(2765)$ and $\Lambda_{c}(2880)$, we will double the errors associated with the fits.

Figure 8.13 shows the $\Lambda_{c}+\pi$ invariant mass. The black data in the first plot 

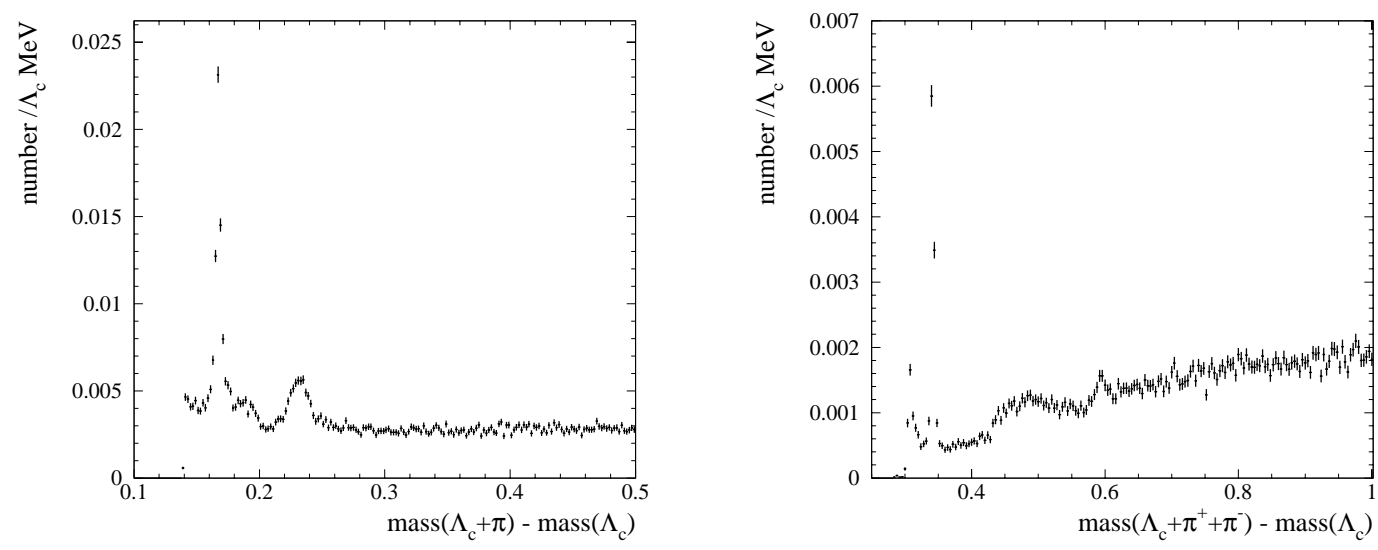

Figure 8.10: Extension of figure 8.9 to higher invariant masses.
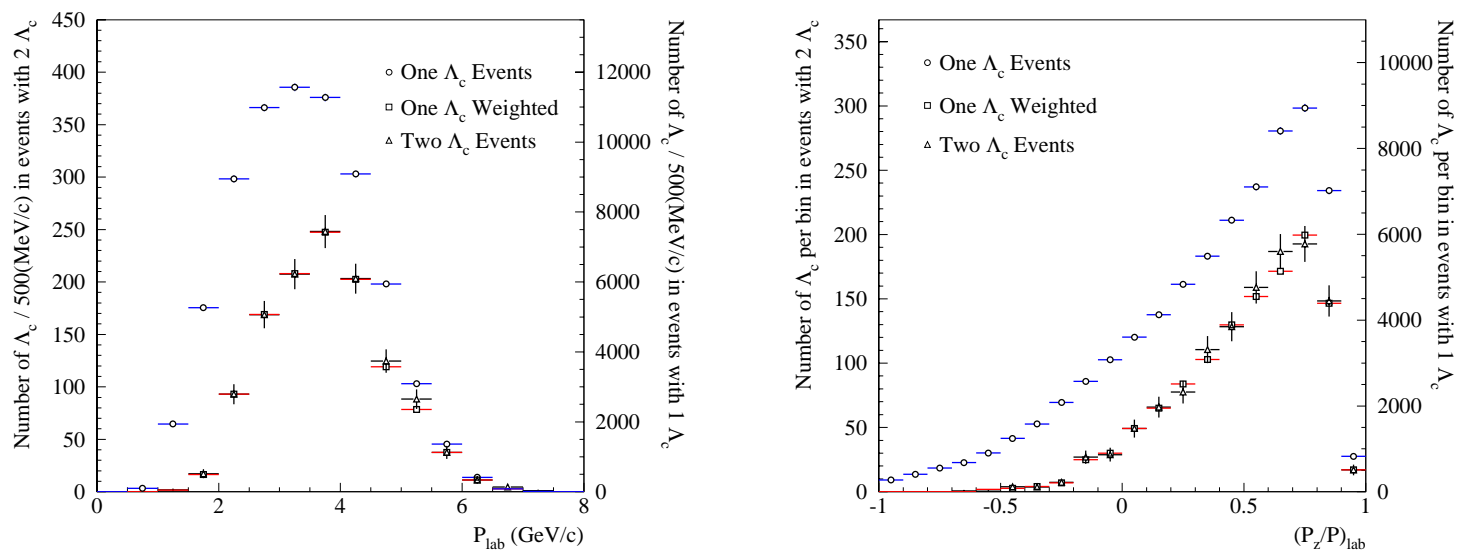

Figure 8.11: The momentum and angle distributions of $\Lambda_{c}$ in one and two $\Lambda_{c}$ events are different. The one $\Lambda_{c}$ events are weighted to match. 

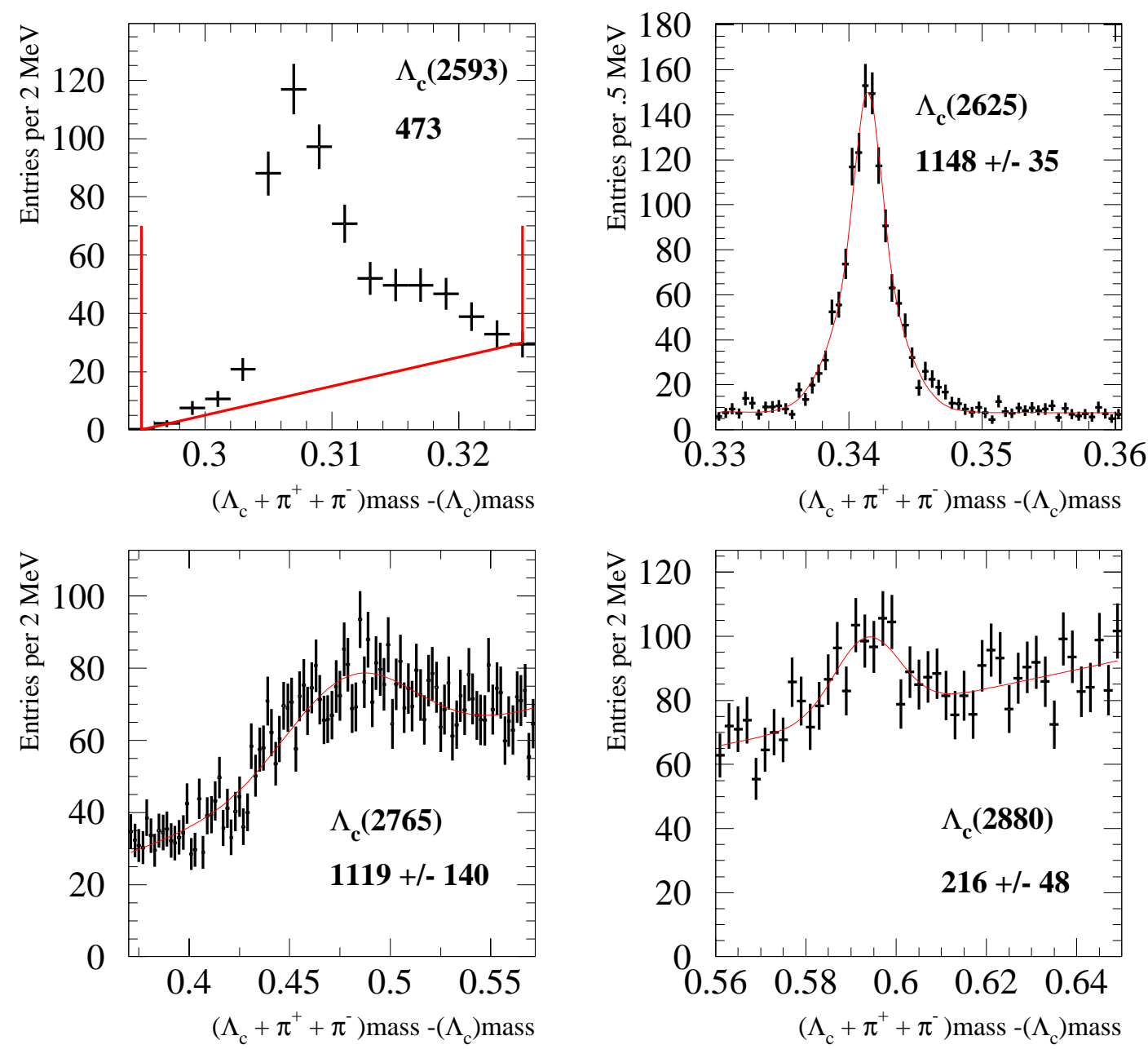

Figure 8.12: $\left(\Lambda_{c}+\pi^{+}+\pi^{-}\right)$invariant mass plots in the regions near four $\Lambda_{c}^{*}$ states. 
has an interesting structure between the $\Sigma_{c}(2455)$ and $\Sigma_{c}(2520)$ peaks. The blue and red plots are the Monte Carlo feeddowns of the $\Lambda_{c}(2625)$ and $\Lambda_{c}(2593)$. The Monte Carlo is scaled to the number of $\Lambda_{c}$ found in figure 8.12 and also weighted to match the momentum vector distribution of the $\Lambda_{c}(2285)$ shown in figure 8.11. When both of the feeddowns are subtracted, we get the plot in the upper right. Now we have an unusual structure to the right of the $\Sigma_{c}(2455)$, which is probably due to the fact that the Monte Carlo $\Lambda_{c}(2593)$ has a Gaussian shape instead of what we saw in figure 8.12. Since this isn't working we will only subtract the $\Lambda_{c}(2625)$ feeddown. The bottom two plots of figure 8.13 show the fits to the $\Sigma_{c}(2455)$ and $\Sigma_{c}(2520)$ after the $\Lambda_{c}(2625)$ subtraction. Both of these are fit with the sum of two Gaussians. In order to account for the unknown effect of the $\Lambda_{c}(2593)$ feeddown, we will double the fit error on the $\Sigma_{c}(2455)$ rate.

Figures 8.12 and 8.13 were made from $32,295 \Lambda_{c}(2285)$ so now we know the observed rate of each heavy charmed baryon per observed $\Lambda_{c}(2285)$. We also need to know the ratio of observed decay pions to observed charmed baryons for each of these decays. For the excited $\Lambda_{c} \rightarrow \Lambda_{c}+2 \pi$, this number is not simply two, because sometimes one of the pion daughters will not be found, meaning that even though we will not see an invariant mass peak, we still will have one of the daughter pions passing the tracking cuts. The number of found pions per found $\Sigma_{c}$ is not necessarily one either, because sometimes the pion will not be reconstructed well enough to show up in the $\Sigma_{c}$ mass region. Again we use Monte Carlo, weighted to match the distributions in figure 8.11 to give us these numbers. The results are summarized in table 8.1. The efficiency errors are added as if they were totally correlated. The fit errors are statistical and are added in quadrature.

Some of the excited $\Lambda_{c} \rightarrow \Lambda_{c}+\pi^{+}+\pi^{-}$states might decay through the intermediate state $\Sigma_{c} \rightarrow \Lambda_{c}+\pi$ so we have to be careful not to double count. PDG 

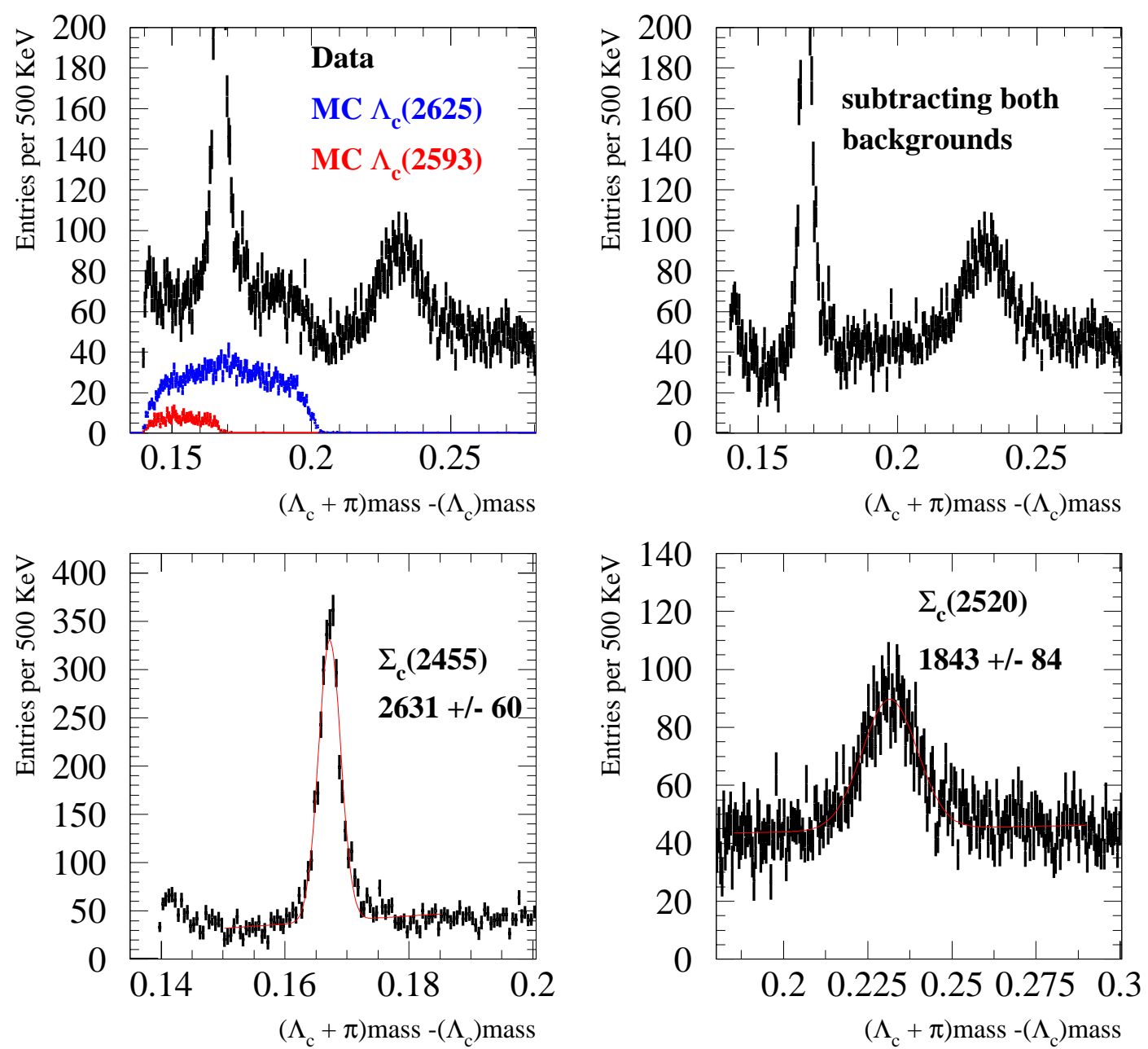

Figure 8.13: $\left(\Lambda_{c}+\pi\right)$ invariant mass plots showing the subtraction of the feeddown from two $\Lambda_{c}^{*}$ states and closeups of the invariant mass regions near two $\Sigma_{c}$ states. 
reports the CLEO result that $72 \pm 15 \%$ of $\Lambda_{c}(2593)$ decay through a $\Sigma_{c}(2455)$. If we assume that the tracking efficiency, E, for both pions is about the same, then for each real $\Lambda_{c}(2593)$ decaying this way we expect to find $E^{2} \Lambda_{c}(2593)$ and $\mathrm{E}$ $\Sigma_{c}(2455)$. So the number of observed $\Sigma_{c}(2455)$ per observed $\Lambda_{c}(2593)$ is $1 / \mathrm{E}$. The pions from the $\Sigma_{c}(2455)$ have already been counted so we have $1 / \mathrm{E}$ extra pions for each reconstructed $\Lambda_{c}(2593)$ decaying in this mode. For this decay $\mathrm{E}=.81$. The other excited $\Lambda_{c}$ states do not seem to decay through a $\Sigma_{c}$ [27].

\begin{tabular}{|l|c|c|c|}
\hline Particle & number observed per $\Lambda_{c}$ & pions per Baryon & detected $\pi$ per $\Lambda_{c}$ \\
\hline$\Sigma_{c}(2455)$ & $.0815 \pm .0037$ & $.98 \pm .04$ & $.0799 \pm .0049$ \\
$\Sigma_{c}(2520)$ & $.0571 \pm .0026$ & $1.14 \pm .08$ & $.0650 \pm .0054$ \\
$\Lambda_{c}(2593)$ & $.0146 \pm .0037$ & $2.47 \pm .10$ & $.0362 \pm .0091$ \\
$\Lambda_{c}(2625)$ & $.0355 \pm .0011$ & $2.66 \pm .24$ & $.0946 \pm .0058$ \\
$\Lambda_{c}(2765)$ & $.0346 \pm .0043$ & $2.82 \pm .21$ & $.0977 \pm .0142$ \\
$\Lambda_{c}(2880)$ & $.0069 \pm .0015$ & $2.76 \pm .14$ & $.0185 \pm .0042$ \\
\hline correction for $\Lambda_{c}(2593) \rightarrow \Sigma_{c}(2455)$ & & $-.0130 \pm .0027$ \\
\hline \multicolumn{2}{|r}{} & total & $.379 \pm .028$ \\
\hline
\end{tabular}

Table 8.1: Summary of the expected number of observed pions from heavier charmed baryon decays.

\subsection{Efficiency Corrections}

In order to calculate the average number of popcorn mesons per event, we need to know the tracking efficiencies, both for popcorn and for the decay pions. A table of tracking efficiencies was made for single $\Lambda_{c}$ Monte Carlo using only pions coming directly from string decay. The relative number of these pions passing 

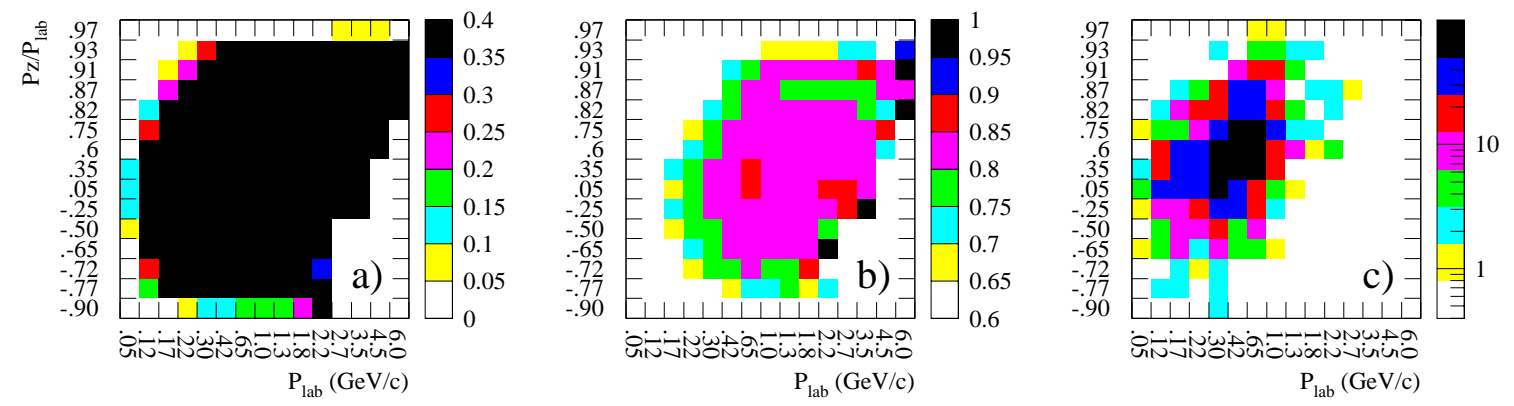

Figure 8.14: Tracking efficiency of pions in $\Lambda_{c}^{+} \mid \bar{\Lambda}_{c}^{-}$events. The z range is modified to better show the edge a) and central b) regions. c) shows the distribution of the pions in the data.

the tracking cuts is shown in figure $8.14 \mathrm{a}$ ) and b). The axis labels correspond to the center point of each bin. Different scales for the z-axis are used to better show the efficiencies near the edge and in the center of the detector. The actual distribution of pions in the data is shown in figure $8.14 \mathrm{c}$ ) and the left plot of figure 8.15. Bins with less than $5 \%$ tracking efficiency or with a lab momentum less than $100 \mathrm{MeV} / \mathrm{c}$ or outside of the angular region $-.90 \leq \cos \theta \leq .94$ will not be used. The number of tracks going into these bins will instead be estimated by extrapolating from adjacent bins.

Running the popcorn candidate distribution shown in figure $8.14 \mathrm{c}$ ) through the correction table gives us a tracking efficiency of $77.7 \pm 3.3 \%$. Only statistical errors are considered. We now need to estimate how many tracks fall outside of the acceptance. The plot on the right of figure 8.15 shows the lab momentum spectrum of the popcorn candidates compared to the true and reconstructed string decay pions in single $\Lambda_{c}$ Monte Carlo. The Monte Carlo distribution is 

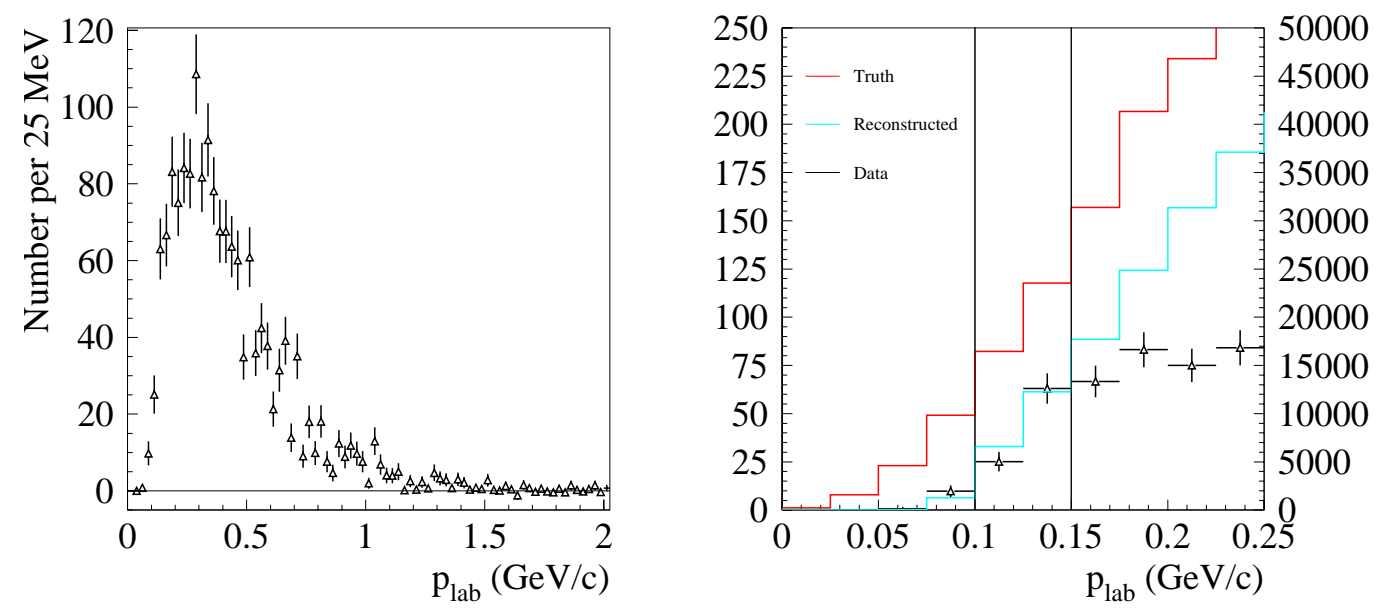

Figure 8.15: The left plot shows the pion momentum distribution in the lab frame. The right plot shows that at low momenta, the slope of this distribution is similar in data and MC. The black lines show the limits of the lowest momentum bin.

much harder than the data but it appears that the slopes of the distributions across the last momentum bin before the edge are similar enough that an extrapolation can be made with an uncertainty of $30 \%$. The extrapolations to the forward and backward angular regions of the detector are easier because the true distributions do not vary as much in these regions. The estimate for the number of data pions in each extrapolated bin of the plots of figure 8.14 is taken to be the number of data pions in an adjacent bin times the ratio of the number of true pions in the extrapolated bin to the number of true pions in the adjacent bin. The result is that we have $175 \pm 52$ pions in the extrapolation region, which lowers our previously calculated tracking efficiency to $71.7 \pm 3.8 \%$.

Since the pions coming from heavy charmed baryon decays will be distributed differently than the popcorn mesons, we will need to estimate their tracking efficiency separately. Using the Monte Carlo to calculate the pion efficiency from 
each of the six heavy charmed baryon decays and then weighting the results in proportion to the number of each decay seen in data, we get a tracking efficiency of $71.5 \pm 1.1 \%$. This is close enough to the result from the data that we do not need to consider any biases due to differing tracking efficiencies between the popcorn and decay pions.

Two adjustments need to be made to the tracking efficiency. One is the subtraction of electrons coming from photon conversions. Neutral pions decay into two photons, which can interact with the detector material to make soft electrons. Our particle identification algorithm was not designed to distinguish electrons from charged pions but we can estimate the number of electrons from photon conversions from Monte Carlo. We do this by taking the single $\Lambda_{c}$ Monte Carlo, and killing off $\pi^{0}$ until the spectrum matches that of the observed charged popcorn pion spectrum from the data (figure 8.15). The spectrum is normalized to one half of the charged pion spectrum, as predicted by isospin invariance. The electron grandaughters of these $\pi^{0}$ are plotted as a percentage of the total number of tracks identified as charged pions in the left plot of figure 8.16. The relative number of electrons in the sample goes up to seven percent in the lowest used momentum bin, but since the total number of tracks is small at low momentum, the total correction is small. After taking into consideration the efficiency and the change in the extrapolation outside the acceptance, we have a correction of -.052 pions per event. Since this is a small correction, we can afford to assign a conservative error of $50 \%$. Of course, the $\pi^{0}$ which create these electrons are popcorn mesons themselves, but we are currently only interested in measuring the charged pions in each event, and so no effort is made to try to include $\pi^{0}$ by reconstucting the photons.

The other tracking correction is charged pion decay into a muon and neutrino. 

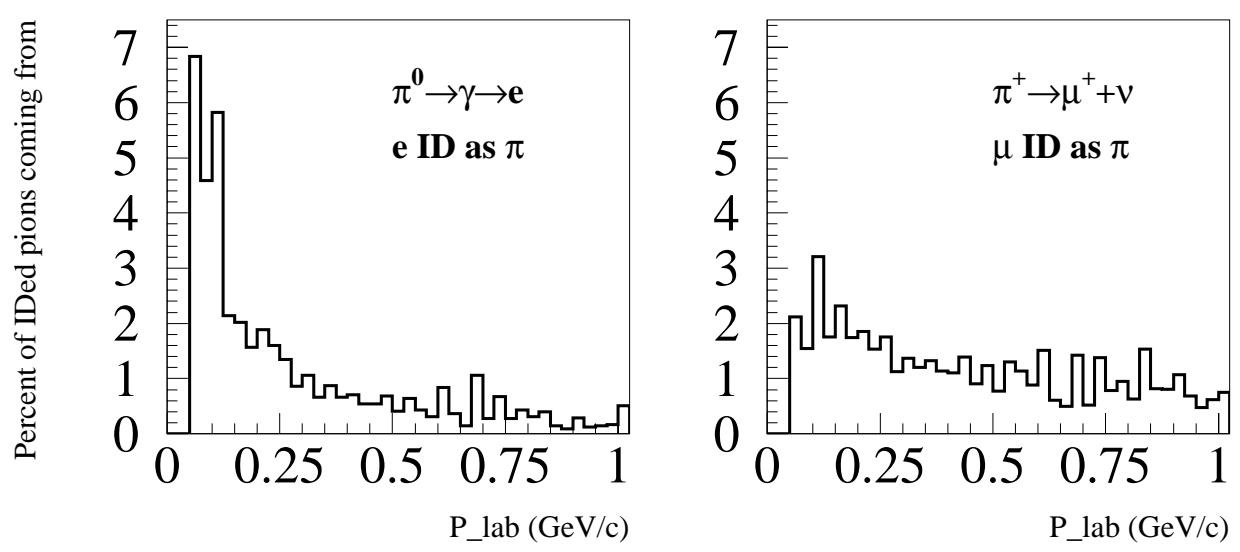

Figure 8.16: Percentage of particles passing the tracking cuts which are not pions. The left plot shows electrons from photon conversions. The right plot shows muons from charged pion decay.

These pions are counted as being lost by the tracking efficiency, but the daughter muons often continue on and pass the tracking cuts, so we need to subtract them to avoid double counting. Again we kill off Monte Carlo particles to match the charged pion momentum spectrum in the data, and see how many of the daughter muons end up passing the tracking cuts. The result is seen in the right plot of figure 8.16. The total correction ends up being -.055 pions per event.

\section{5 $\quad K_{0}, \rho$ and $\omega$}

The study of neutral popcorn mesons decaying to $\pi^{+}+\pi^{-}$is interesting for two reasons. First of all, since $K_{0}$ can have flight lengths of several centimeters, their daughters might not pass our tracking cuts, resulting in an underestimation of the amount of popcorn. Second, the multiplicities of heavier mesons might be 

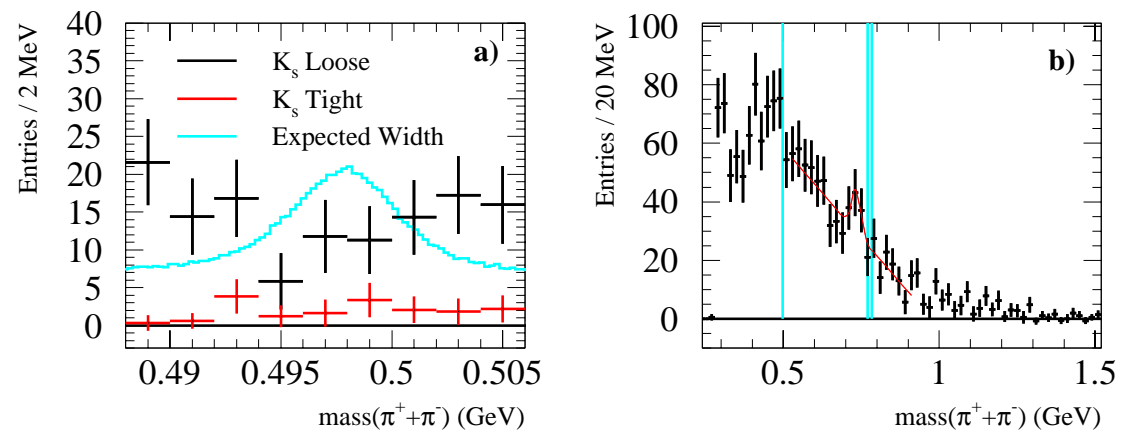

Figure 8.17: a) $K_{s}$ candidates in $\Lambda_{c}^{+} \mid \bar{\Lambda}_{c}^{-}$events. b) $\left(\pi^{+} \pi^{-}\right)$invariant mass. The light blue lines show the locations of the $K_{s}, \rho$ and $\omega$ masses.

relatively easily measureable quantities that could be compared with the various model predictions.

The black points in figure 8.17a show the invariant mass of $K_{0}$ candidates satisfying the "initial cuts" corresponding to figure 4.9; two oppositely charged tracks with more than 10 DCH hits, $0 \leq$ flightlength $(\mathrm{cm}) \leq 60$, vertex probability $\geq 1 \%$. The red points require the additional cuts of a flight length of greater than $2.5 \mathrm{~mm}$, and a cosine of greater than .97 for the angle between the flightlength and momentum vectors. There doesn't appear to be any $K_{0}$ signal. Isospin invariance predicts an equal number of charged and neutral kaons. Since we only observe the $K_{s}$ we would expect to see about 23, times the $K_{s} / K^{+}$efficiency ratio, if all 46 of the observed charged kaons were real. This suggests that a large number of the observed $K^{+}$might be misidentified pions.

The black points in figure $8.17 \mathrm{~b}$ show the invariant mass of all $\pi^{+}+\pi^{-}$combinations for which both pions pass our tracking cuts. Note that this is different from the $K_{s}$ candidates shown in figure 8.17a, where the daughter tracks are not 
required to come from the event vertex. The vertical blue lines mark the masses of the $K_{0}, \rho$, and $\omega$. Interference between $\rho$ and $\omega$ can create a distribution to the left of $\rho$ mass, similar to what we see. Fitting this bump with a Gaussian plus a linear background gives us $32 \pm 13$ entries, but more statistics will be needed to get a reliable measurement of the $\rho$ and $\omega$ rates. For now we just count these as two popcorn pions.

The peak near threshold in figure $8.17 \mathrm{~b}$ is what one would typically expect from photon conversions. However, given the number of photon conversions predicted by Monte Carlo (fig. 8.16), we only expect four entries in this peak. In addition, reducing the required distance of closest approach from $5 \mathrm{~mm}$ to $1 \mathrm{~mm}$ does not reduce the size of the peak, as would be expected if it were due to photon conversions. It may be that the peak is due to statistical fluctuations.

\subsection{Results}

This section will give our result for the number of charged mesons, in $\Lambda_{c}^{+} \mid \bar{\Lambda}_{c}^{-}$ events with no extra baryons, which are not daughters of the six heavy charmed baryon decays described in section 8.3. The possibility of distinguishing popcorn mesons from the daughters of as of yet undiscovered heavy charmed baryons will be addressed in the next section.

In order to reduce as much as possible the number of events in our sample with extra baryons, we have thrown out all events with either an observed proton, or with a missing mass or non- $\Lambda_{c}$ mass less than $-500 \mathrm{MeV}$. In order to limit the tracking correction applied to any particular track we throw out any observed track which hits the detector in a region with less than 5\% tracking efficiency, and also any track which ends up in the first column, or the first or last row, of 

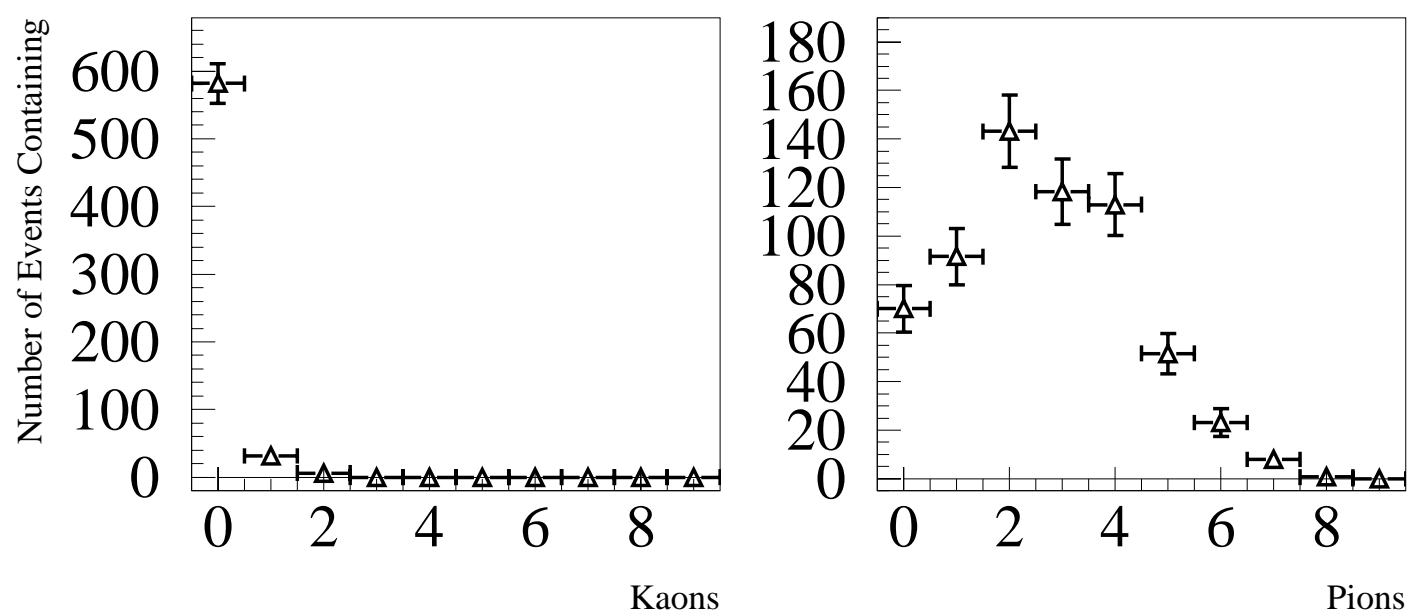

Figure 8.18: Number of kaons and pions per event after all cuts.

the tracking matrix shown in figure 8.14.

These cuts leave us with 1641 pions in 619.3 events - 2.65 pions per event. There are also 43.6 charged kaons. The number of kaons and pions in each event is shown in figure 8.18. In order to estimate the uncertainty on the number of pions per event, we run 10,000 toy Monte Carlos, each with 619 signal events, 3240 background events weighted with a factor of $1 / 12$, and a Poissonian distribution of pions with mean 2.65 in each event. The width of the resulting pion per event distribution is .078.

As a cross check we divide the data into 5 regions. We use a circular signal region with radius $2.5 \mathrm{MeV}$, and 4 contiguous concentric rings with outer radii of 4, 6, 8.5 and $16 \mathrm{MeV}$. The result from each of these regions is shown in figure 8.19. Again the errors are calculated using toy Monte Carlos.

The corrections and uncertainties due to different sources are tabulated in 


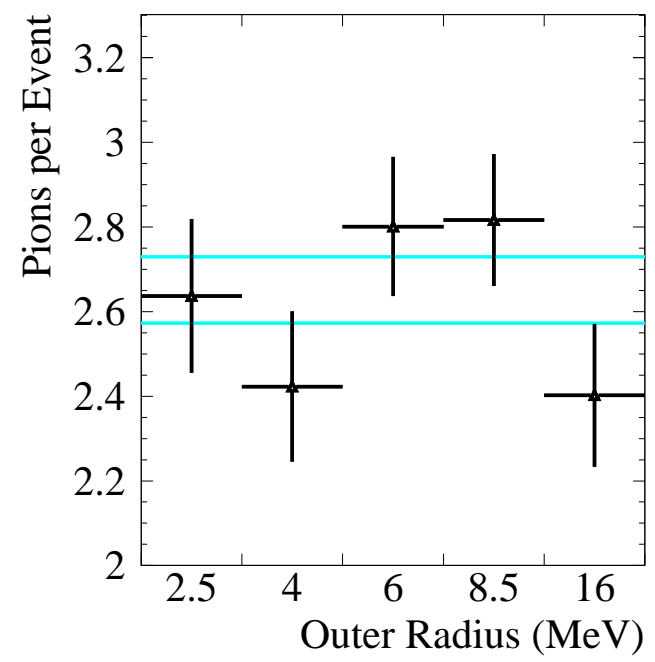

Figure 8.19: Number of charged pions per event for five different signal regions made up of contiguous rings of varying outer radii. The blue lines show the uncertainty of the measurement using the signal region consisting of a $12 \mathrm{MeV}$ radius circular region. 
table 8.2. First the pions from decays of heavy charmed baryons are subtracted. Because we expect only 469 decay pions in our sample, a relative counting error of $1 / \sqrt{469}$ is added in quadrature to the value from table 8.1.

Then an adjustment is made for events with either two neutrons or with unseen protons. Because these events have fewer pions than the events with no extra baryons, subtracting them raises our pion per event result slightly. We estimate that there are $5 \pm 5$ of these events. We also make a correction for tracks which are reconstructed twice.

At this point the kaons are added back in with a correction for the large amount of pion as kaon misidentification. Because we do not expect to see any baryon decaying to $\Lambda_{c}+\mathrm{K}$, kaons that are believed to be real are all counted as popcorn mesons. However, a pion misidentified as a kaon has about a $28 \%$ probability of having come from one of the decays described in section 8.3. Based on the fact that we saw a larger amount of pion as proton misidentification in the data than in the Monte Carlo, we also expect to see more pion as kaon misidentification. We therefore estimate that $20 \pm 15$ of the tracks identified as kaons are actually pions, and the remaining 23 are kaons.

These corrections give us a subtotal of $1.96 \pm .10$ charged mesons observed per event. Dividing by a tracking efficiency of .717 \pm .038 and then subtracting the photon conversions and charged pion decays gives us a final result of $2.63 \pm$ .21 per event. Statistics account for .08 of this. The largest error is the tracking efficiency, which is difficult to estimate near the detector edges because of low statistics.

Isospin invariance predicts that we will see $50 \%$ as many neutral pions as charged pions. This is not exactly right, because for a particular number of popcorn mesons, only certain charge combinations are possible. For example, if 
we have only one popcorn pion, it must be a neutral $\pi^{0}$. Nevertheless, we expect that there are roughly 1-2 $\pi^{0}$ per event, giving a total of about 4 popcorn mesons per event. This is much larger than the .5 predicted with the current JETSET tuning.

In the next section, we will try to determine how many of these mesons could be coming from decays of heavier charmed baryons resonances that have not yet been discovered.

\begin{tabular}{|c|r|}
\hline Source & Mesons per Event \\
\hline Measured Pions & $2.651 \pm .078$ \\
Pions from Known Decays & $-.758 \pm .066$ \\
4 Baryon Events & $.016 \pm .016$ \\
Double Counted Tracks & $-.011 \pm .000$ \\
Kaons & $.061 \pm .007$ \\
\hline Subtotal & $1.959 \pm .104$ \\
\hline$\div$ Tracking & $71.7 \pm 3.8 \%$ \\
& $2.732 \pm .201$ \\
$\pi \rightarrow \mu$ & $-.052 \pm .026$ \\
$\gamma \rightarrow e$ & $-.055 \pm .028$ \\
\hline Total & $2.625 \pm .205$ \\
\hline
\end{tabular}

Table 8.2: Summary of the number of charged pions and kaons in $\Lambda_{c}^{+} \mid \bar{\Lambda}_{c}^{-}$events with no extra baryons and which are not daughters of known charmed baryon resonances. 


\subsection{Decays of Undiscovered Charmed Baryons.}

In section 8.3 we studied the decays of the previously discovered $\Sigma_{c}$ and $\Lambda_{c}$ states in order to estimate the amount of background they contributed to our popcorn measurement. However, there potentially could be a large number of as-of-yet undiscovered charmed baryons, which may be contributing to our signal.

We would like to come up with a way to distinguish these decays from true popcorn. One possibility is to look at the helicity angles of the decays. When a $\Sigma_{c}$ decays strongly to a $\Lambda_{c}(2285)+\pi$, we expect that in the rest frame of the $\Sigma_{c}$, the distribution of the angle between the pion momentum and the $\Sigma_{c}$ momentum will be front/back symmetric. The same is true if we combine the $\Lambda_{c}(2285)$ from a $\Lambda_{c}^{*} \rightarrow \Lambda_{c}(2285)+2 \pi$ decay with either of the two daughter pions.

If, however, we calculate the same angle by combining a $\Lambda_{c}$ with a popcorn pion, we will get a backward sloping distribution. Because popcorn mesons are

created in between the $\Lambda_{c}^{+}$and $\bar{\Lambda}_{c}^{-}$, we expect to see the pion moving backwards, relative to the $\Lambda_{c}$.

The plots showing these angular distributions are shown in figure 8.20. The first plot shows the data. In order to prevent getting an artificially backwards sloping plot caused by associating a pion with the $\Lambda_{c}$ from the opposite hemisphere, each pion is combined only with the $\Lambda_{c}$ with which it forms the lowest invariant mass. The black distribution is for all pions which are not $\Lambda_{c}$ daughters and which pass all the cuts described in the previous section. The red points represent the pions from all six decay backgrounds, which are shown individually in the next six plots. As expected, the data is backwards sloping and the background is close to being flat. The dropoff in the most backward bins is caused by the fact that the backward moving tracks are slower, and thus less likely to pass 
the tracking cuts.

The bottom center plot shows the background subtracted signal. The symmetric part of the distribution appears to be very small, which would suggest that only a small percentage of these pions could be coming from a charmed baryon decay that has not yet been discovered. A problem occurs, however, when we look at the angular distribution of pions from a hypothetical, high mass charmed baryon. The bottom right plot of figure 8.20 shows that the distribution of helicity angles for the hypothetical decay $\Lambda_{c}(3300) \rightarrow \Lambda_{c}(2285)+2 \pi$ has a backward peaked asymmetry that is similar to what we expect for popcorn, but when we plot the angle for all generated $\Lambda_{c}(3300)$ in red, we see that the distribution is flat, as expected. What is happening is that when a pion decays in front of the baryon, the daughter $\Lambda_{c}$ ends up moving slower, and thus is less likely to pass the minimum $2.3 \mathrm{GeV} / \mathrm{c}$ momentum cut.

If the high mass continua in figure 8.10 actually contain overlapping broad excited charmed baryons states, they could contribute backward peaked mesons such as we observe. Two things should be noted, however. First, the asymmetic helicity angle plots will only occur when the decay pions have a large momentum relative to the $\Lambda_{c}(2285)$. If the heavy charmed baryon decays in a series of steps, each of which resulting in a slow daughter pion, or if it decays to several pions at once in such a way as to limit the probability that any one will have a large relative momentum, then the helicity angles plots will be flat. Second, since the known heavy charmed baryons produce on average 0.06 observed decay pions per observed $\Lambda_{c}$, and we observe just less than one popcorn meson per $\Lambda_{c}$, one would expect that at least 16 such heavy charmed baryons would have to exist in order to explain our data as being solely due to decays. However, since most models predict that heavier resonances should be produced at lower rates, we 
would expect the rates to get progressively smaller as we go up in mass, meaning that many more than 16 of these resonances would be required.

Even if these undiscovered heavy resonances did exist, we still have arguments leading to the conclusion that at least one popcorn meson would still be present in each event. In order to get no popcorn, we would have to have the virtual photon going directly into two charmed baryons, with no additional particles. These baryons would have masses between about $3.3 \mathrm{GeV}$, the minimum mass necessary to produce the asymmetry we see in the popcorn condidates, and 5.2 $\mathrm{GeV}$, the kinematic limit for BaBar. If these baryons were near the upper mass limit, we would have a situation that kinematically would be very similar to the $\Upsilon(4 S) \rightarrow B^{0} \bar{B}^{0}$ events. The heavy baryons would be near rest in the center of mass frame, meaning that they would decay isotropically. This is clearly not happening as we see in top right plot of figure 8.5 that the $\Lambda_{c}$ are produced back to back.

As the heavy charmed baryon-antibaryon pair gets lighter, the events become more jetlike, making the $\Lambda_{c}$ more and more back to back. However, we expect the rates for such baryon-antibaryon production to fall with mass. Studies of exclusive proton-antiproton production shows that the cross section for such events falls rapidly for invariant masses more than $350 \mathrm{MeV}$ above proton-antiproton threshold[29]. In our data, we see in the bottom right plot of figure 8.5 that we have no events with a $\Lambda_{c}^{+} \mid \bar{\Lambda}_{c}^{-}$and nothing else. So we do not expect events with $\Sigma_{c}^{+} \mid \bar{\Sigma}_{c}^{-}$or $\Lambda_{c}^{+*} \mid \bar{\Lambda}_{c}^{-*}$ and nothing else either.

So, in conclusion:

- We find $2.63 \pm .21$ additional charged mesons in each event, after subtracting decay pions from the six known charmed baryon resonances decaying to a $\Lambda_{c}$ 
- We do not see any evidence for new charmed baryons in the invariant mass plots (figure 8.10).

- If undiscovered resonances existed at the same average rate as the ones we already see, there would need to be about 16 new states to explain all of the data.

- All hadronization models predict that production rates should fall with increased mass, meaning many more than 16 states would be required.

- The fact that exclusive baryon-antibaryon production is very small far above threshold, and, that production near threshold would not result in the jet-like events that we see seems to imply that at least one popcorn meson is present in every event. 

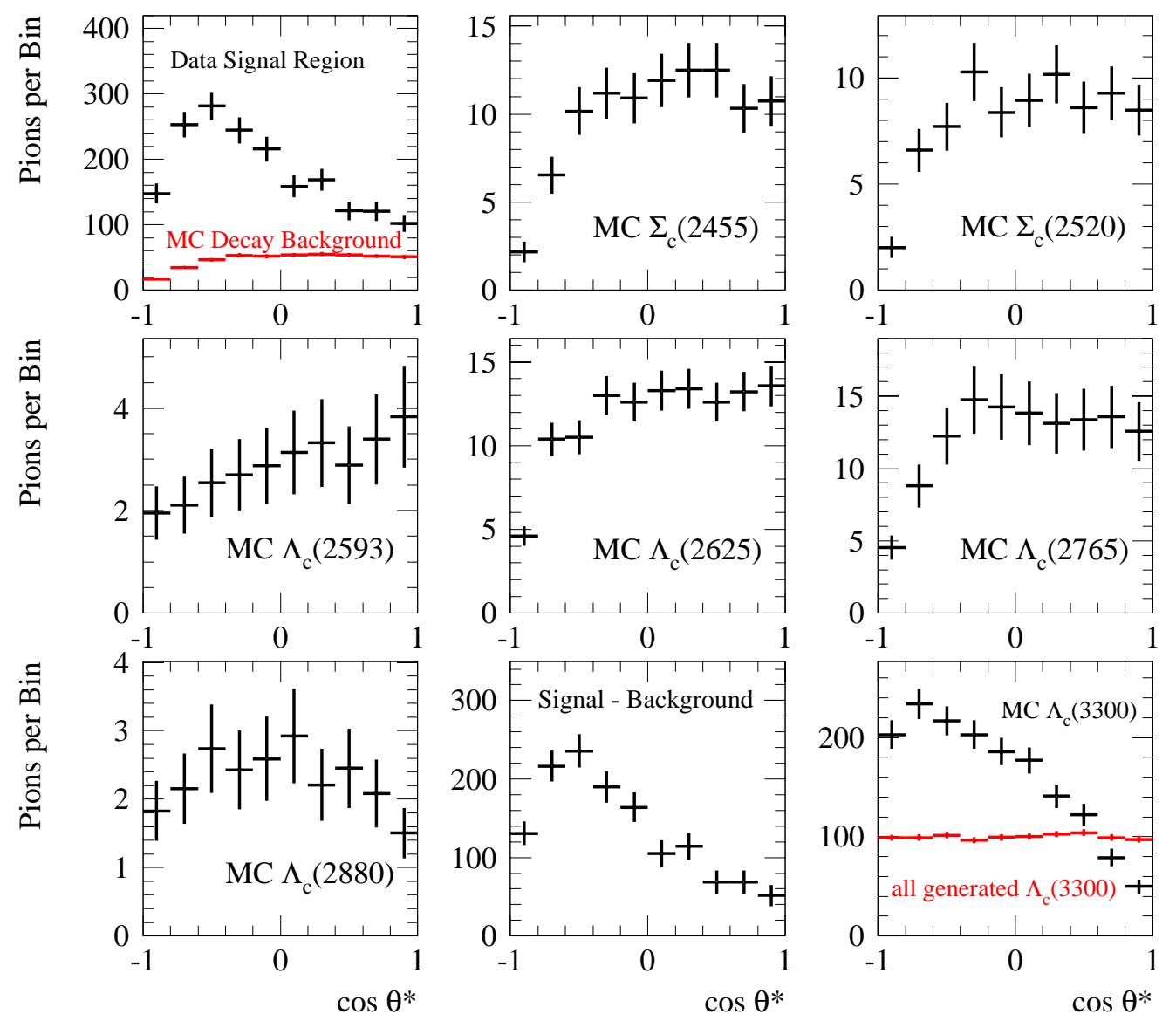

Figure 8.20: Cosine of the angle between the $\pi$ and $\left(\Lambda_{c}+\pi\right)$ momenta in the $\left(\Lambda_{c}+\pi\right)$ rest frame for data, and for the six known decays to $\Lambda_{c}+\mathrm{N} \pi$. Also included are the plots for background subtracted signal and the decay of the hypothetical particle $\Lambda_{c}(3300)$. 


\section{CHAPTER 9}

\section{Conclusion}

This analysis was divided into two parts, the first being the measurement of the continuum $\Lambda_{c}$ momentum spectrum at $10.54 \mathrm{GeV} / \mathrm{c}$, and the second being the search for popcorn mesons in continuum events with a $\Lambda_{c}^{+} \bar{\Lambda}_{c}^{-}$pair.

The continuum spectrum measurement was carried out on a data sample of $9.460 \mathrm{fb}^{-1}$ at a center of mass energy of $10.54 \mathrm{GeV}$. After taking into account acceptance losses, this represents a 13-fold increase in data over the previous best $\Lambda_{c}$ momentum spectrum at this energy.

Our results were compared to nine different fragmentation models. In general the two parameter models - Lund, Bowler and UCLA - outperformed the one parameter models, most of which were unable to reproduce the narrowness of the peak of the distribution. The exception was the Modified Kartvelishvili function, which unfortunately was the only function that was easily modifiable to predict baryon instead of meson spectra. It was found that the rates of charmed baryons decaying into $\Lambda_{c}$ could change the model spectrum predictions. It would be an interesting future study to tune each of the models to the best available charmed baryon multiplicities, in order to see the effect on the $\Lambda_{c}$ momentum spectrum predictions.

The second part of the analysis looked for events with a $\Lambda_{c}^{+} \bar{\Lambda}_{c}^{-}$pair. Very few of these events contained additional protons or kaons, but a large number 
of additional pions were found. After subtracting the background from the six particles known to decay to a $\Lambda_{c}+$ pions, we were left with $2.63 \pm .21$ additional charged pions and kaons per event. We expect roughly 1-2 more neutral mesons per event.

The fact that so few additional protons are observed in these events demonstrates that baryon number does not have to be conserved locally within each jet as the present implementations of the Monte Carlos require. If JETSET were able to generate events with long range baryon correlations and the current popcorn parameter were unchanged, it would generate an average of .5 popcorn mesons per $\Lambda_{c}^{+} \mid \bar{\Lambda}_{c}^{-}$event. The UCLA model allows for a distribution of popcorn masses, a large portion of which is above $1 \mathrm{GeV}$. This approach, if it could be adapted to long range baryon correlations, would provide a more accurate modeling of events with multiple popcorn mesons.

We have experimentally established that there are about 2.6 additional charged mesons per event. If each of these mesons is combined with the $\Lambda_{c}$ that it is closest to, the angle of the meson's momentum in the rest frame of the combined object is more likely to be pointing back toward the center of the event. This seems to suggest that these mesons are popcorn.

An alternative model would be that these mesons are decay products of heavy charmed baryons and that the asymmetry of this angular distribution is caused by the large recoil of the forward decaying mesons pushing the $\Lambda_{c}$ daughter back below our $2.3 \mathrm{GeV} / \mathrm{c}$ center of mass momentum cut. It seems unlikely, however, that enough of these high mass states could exist to be able to increase our observed number of decay pions by the factor of 2.5 necessary to explain all of the data.

The jetlike geometry of our events rules out the possibility of the exclusive 
production of a very heavy baryon-antibaryon pair decaying into $\Lambda_{c}^{+} \bar{\Lambda}_{c}^{-}+N \pi$. Since we expect the cross section for lighter exclusive baryon-antibaryon states to be very low, we have strong evidence that at least one popcorn meson is present in the vast majority of our events. 


\section{REFERENCES}

[1] CLEO Collaboration, A.Bornheim et al., Correlated $\Lambda_{c}^{+} \bar{\Lambda}_{c}^{-}$production in $e^{+} e^{-}$ annihilations at $\sqrt{s} 10.5 \mathrm{GeV}$ Phys. Rev. D 63 (2001)

[2] Quarks and Leptons Francis Halzen and Alan D. Martin, Joh Wiley and Sons, Inc. (1984).

[3] W. Feilmair, H. Markum, Nuclear Physics B370 (1992) 299.

[4] The Lund Model B. Andersson, Cambridge Monographs on Particle Physics, Nuclear Physics and Cosmology, Vol 7 (1998).

[5] Introduction to Elementary Particles David Griffiths, John Wiley and Sons, Inc. (1987).

[6] B. Andersson, G. Gustafson, G. Ingelman, T. Sjöstrand, Phys. Rep. 97 (1983) 32.

[7] C. Peterson, D. Schlatter,I. Schmitt and P.M. Zerwas Phys. Rev. D27 (1983) 105.

[8] S.J. Brodsky, J.F. Gunion, Phys. Rev.D17 (1978) 848.

[9] V.G. Kartvelishvili, A.K. Likhoded and V.A. Petrov,, Phys. Lett. C78B (1978) 615.

[10] M.G. Bowler, Z. Phys. C11 (1981) 169.

[11] P.D.B Collins and T.P. Spiller, J. Phys. G 11 (1985) 1289.

[12] E. Braaten, K. Cheung, S. Fleming, T.C. Yuan, Phys. Rev. D 51 (1995) 4819.

[13] G. Marchesini and B.R. Weber, Nucl. Phys. B238 (1984) 1.

[14] S. Chun and C. Buchanan, A simple plausible path from QCD to successful prediction of $e^{+} e^{-} \rightarrow$ hadronization data, Phys. Rep. 292 (1998) 239-317.

[15] S. Abachi and C. Buchanan, The UCLA Space-Time Area Law Model; A persuasive Foundation for Hadronization, to be published.

[16] A. Casher, H. Neuberger and S. Nussinov, Phys. Rev.D20 (1979) 179.

[17] OPAL Collaboration, P.D. Acton, et al., Phys. Lett. B305 (1993) 415. 
[18] DELPHI Collaboration, P. Abreu et al., Phys. Lett. B318(1993) 249.

[19] DELPHI Collaboration, P. Abreu et al., Phys. Lett. B416 (1998) 247.

[20] BABAR Collaboration, B. Aubert et al., Nucl. Instrum. Meth. A479 (2002) $1-116$.

[21] Internal BaBar document, Margaret Haire, BAD 801

[22] S. Christ, Ph.D. Thesis, Rostock University, "Inclusive hadronic spectra of $B^{0}$ and $B^{+}$using fully reconstructed B-meson decays" (2003)

[23] CLEO Collaboration, R.A. Briere et al., Measurements of charm fragmentation into $D_{s}^{*+}$ and $D_{s}^{+}$in $e^{+} e^{-}$Annihilations at $\sqrt{s}=10.5 \mathrm{GeV}$, Phys. Rev. D $62(2000)$

[24] CLEO Collaboration, P. Avery et al., Inclusive production of the charmed baryon $\Lambda_{c}^{+}$from $e^{+} e^{-}$annihilations at $\sqrt{s}=10.55$ GeV Phys. Rev. D 43 (1991) 3599-3609

[25] ARGUS Collaboration, H. Albrecht et al., Observation of the Charmed Baryon $\Lambda_{c}$ in $e^{+} e^{-}$Annihilations at 10 GeV, Phys. Lett. B 207 (1988) 109114

[26] Study of double charmonium production in $e^{+} e^{-}$Annihilations at $\sqrt{s}=10.6$ GeV, K. Abe et al, Phys. Rev. D70 (2004)

[27] Internal BaBar document, Margaret Haire, BAD 801

[28] A. Blechman, A. Falk, D. Pirjol, Phys. Rev. D67 (2003) 074033

[29] Internal BaBar document, Vladimir Druzhinin, Sergey Serednyakov and Evgeni Petrovich, BAD 855 